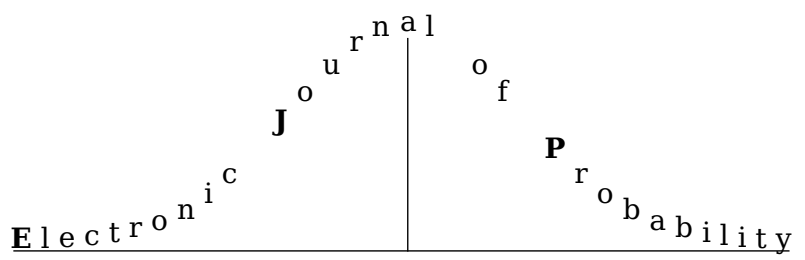

Electron. J. Probab. 26 (2021), article no. 114, 1-42.

ISSN: 1083-6489 https://doi.org/10.1214/21-EJP683

\title{
Infinite dimensional pathwise Volterra processes driven by Gaussian noise - Probabilistic properties and applications -*
}

\author{
Fred Espen Benth ${ }^{\dagger} \quad$ Fabian A. Harang ${ }^{\ddagger}$
}

\begin{abstract}
We investigate the probabilistic and analytic properties of Volterra processes constructed as pathwise integrals of deterministic kernels with respect to the Hölder continuous trajectories of Hilbert-valued Gaussian processes. To this end, we extend the Volterra sewing lemma from [18] to the two dimensional case, in order to construct two dimensional operator-valued Volterra integrals of Young type. We prove that the covariance operator associated to infinite dimensional Volterra processes can be represented by such a two dimensional integral, which extends the current notion of representation for such covariance operators. We then discuss a series of applications of these results, including the construction of a rough path associated to a Volterra process driven by Gaussian noise with possibly irregular covariance structures, as well as a description of the irregular covariance structure arising from Gaussian processes time-shifted along irregular trajectories. Furthermore, we consider an infinite dimensional fractional Ornstein-Uhlenbeck process driven by Gaussian noise, which can be seen as an extension of the volatility model proposed by Rosenbaum et al. in [13].
\end{abstract}

Keywords: infinite dimensional stochastic analysis; Hilbert space; Gaussian processes; covariance operator; Volterra integral equations; rough path integration; fractional differential equations; rough volatility models.

MSC2020 subject classifications: 60H05; 60H20; 45D05; 34A12.

Submitted to EJP on May 29, 2020, final version accepted on August 7, 2021.

Supersedes arXiv:2005.14460.

${ }^{*}$ The authors gratefully acknowledge financial support from the STORM project 274410, funded by the Research Council of Norway.

${ }^{\dagger}$ University of Oslo, Department of Mathematics, POBox 1053 Blindern, N-0316 Oslo, Norway.

E-mail: fredb@math.uio.no

${ }^{\ddagger}$ University of Oslo, Department of Mathematics, POBox 1053 Blindern, N-0316 Oslo, Norway.

E-mail: fabianah@math . uio.no 


\section{Introduction}

Volterra processes appear naturally in models with non-local features. In this article, we will investigate the analytic and probabilistic properties of Volterra processes constructed as pathwise integrals of a kernel $K$ against a Gaussian process $W$. For generality, we will assume that the Gaussian process $W$ takes values in a Hilbert space $H$ with a covariance operator $Q_{W}$, and that the kernel $(t, s) \mapsto K(t, s)$ for $t>s$ is a linear operator on the same Hilbert space. In particular, we define the process $X:[0, T] \rightarrow H$ formally by the integral

$$
X(t)=\int_{0}^{t} K(t, s) d W(s) .
$$

At a discrete level, one can think of this process as assigning different weights through the kernel $K$ to the increments of $W$. Volterra processes have received much attention in the field of stochastic analysis over the past decades. The canonical examples are the Ornstein-Uhlenbeck process where $K(t, s)=\exp (-\alpha(t-s))$ and $W$ a Brownian motion, or the fractional Brownian motion where $K(t, s)=(t-s)^{H-\frac{1}{2}}$ and $W$ is a Brownian motion. These processes are typically used to model phenomena where some sort of memory is inherent in the dynamics, and applications are found in various fields ranging from physics and turbulence modelling [3] to biology [25] and financial mathematics $[15,4]$. See also [2] and the references therein for an introduction to these processes and their applications.

In order to make sense of the integral appearing on the right-hand side of (1.1), one must assume some type of regularity conditions on $K$ and $W$. The type of regularity conditions needed, typically depends on the choice of integral that is used in the construction of $X$. For example, if $W$ is a $Q_{W}$-Wiener process (the infinite dimensional extension of the classical Brownian motion) one would need that $K$ is (Bochner) square integrable in time $s \mapsto K(t, s)$ up to (and including) $t$ (see e.g. [21]). However, for general real-valued Gaussian processes, this is not a sufficient criterion. Indeed, in the case of general Gaussian processes on the real line, it is well known that the Volterra processes appearing in (1.1) makes sense as a Wiener integral if

$$
\int_{0}^{T} \int_{0}^{T} K(T, r) K\left(T, r^{\prime}\right) \frac{\partial^{2}}{\partial r \partial r^{\prime}} Q_{W}\left(r, r^{\prime}\right) d r d r^{\prime}<\infty,
$$

where $Q_{W}$ is the real valued covariance of the Gaussian process $W$ (see e.g. [19]). This construction requires of course that $Q_{W}$ is differentiable in both variables, or at least of bounded variation simultaneously in both variables, which excludes several interesting Gaussian processes (particular examples of which will be discussed in detail later). An extension of the above condition to the infinite dimensional setting when $W$ is an Hilbertvalued process is quite straightforward, but one would still require strong regularity of the covariance operator $Q_{W}$ (say Fréchet differentiable). In several interesting examples, such regularity requirements on the covariance operator are too strong. For example, consider a Gaussian processes $(B(t))_{t \in[0, T]}$ time-shifted along an irregular (possibly deterministic) path $(Z(t))_{t \in[0, T]}$, given as the composition process $(B(Z(t)))_{t \in[0, T]}$. The regularity of the covariance would typically be given as the composition of the regularity of the covariance associated to $B$ and the regularity of $Z$. Thus if $t \mapsto Z(t)$ is only Hölder continuous, one would not expect to get better regularity of the covariance than that of $Z$. The canonical example of such processes is the iterated Brownian motion given as

$$
\mathbb{B}\left(t, \omega_{1}, \omega_{2}\right)=B^{1}\left(\omega_{1},\left|B^{2}\left(\omega_{2}, t\right)\right|\right)
$$

where $B^{1}:[0, T] \times \Omega_{1} \rightarrow \mathbb{R}$ and $B^{2}:[0, T] \times \Omega_{2} \rightarrow \mathbb{R}$ are two independent Brownian motions on the real line. Such processes have received much attention due to their 
curious probabilistic properties, as well as applications towards modelling of diffusions in cracks $[24,9,10]$. If we now fix a trajectory of $B^{2}$, it is readily seen that $\mathbb{B}\left(\cdot, \omega_{2}\right)$ is Gaussian, with covariance given by

$$
Q_{\mathbb{B}\left(\omega_{2}\right)}(t, s)=\min \left(B^{2}(t, \omega), B^{2}(s, \omega)\right),
$$

and therefore the regularity of $Q_{\mathbb{B}}$ is inherited by the regularity of $t \mapsto B^{2}(t, \omega)$. Hence, the covariance function associated to a Volterra process driven by $\mathbb{B}\left(\cdot, \omega_{2}\right)$ can not be constructed as in (1.2), but an extension of this construction is needed.

In recent years, pathwise analysis of stochastic processes has become prevalent in the literature. This plays a fundamental role when applying these processes in the theory of rough paths $[22,14]$, where analytic properties of the paths and associated "iterated integrals" constitute the main ingredients. The advantage of the rough path theory lies in the flexibility to construct pathwise solutions to controlled ODEs on the form

$$
d Y(t)=f(Y(t)) d X(t), \quad Y(0)=y \in H,
$$

even when $X$ is not a semimartingale. Furthermore, one directly obtains stability in the solution mapping $(X, y) \mapsto \Gamma(X, y)$ induced by the equation above. The rough path theory opens for considering equations controlled by noise given as Volterra processes, which typically is of a non-semimartingale nature due to the kernel $K$. Much work has therefore been devoted to the construction of the so-called rough path above a given Volterra process driven by a Brownian motion [23,33]. On the other hand, to the best of our knowledge, there is no construction of the rough path above Volterra processes driven by Gaussian noise with irregular covariance structures. In [14, Sec. 10.2] the authors provide a simple criterion for the existence of a geometric rough path connected to a given Gaussian process, given that the covariance structure of this process is sufficiently regular. This requires of course the existence of a covariance function, which in the case of Volterra processes driven by Gaussian noise is given by (1.2). A relaxation of the existence criteria for (1.2) to the case of non-smooth covariances $Q_{W}$ and with singular Volterra kernels $K$ is therefore needed in order to construct the rough path associated to this class of processes.

The main goal of this article is therefore to extend the sufficient conditions for construction of the covariance operator on the form of (1.2) to the case when $W$ is an infinite dimensional stochastic process and $Q_{W}$ is possibly nowhere differentiable in both variables. To this end, we start by giving a pathwise description of the Volterra process $X$ stated in (1.1). Given a sample path of a Gaussian process $W$ which is $\alpha$-Hölder regular, we will show that (1.1) can be constructed in a pathwise sense through a slight modification of the newly developed Volterra Sewing Lemma from [18]. In this way, one directly obtains the regularity of the process $X$ as the composition of the possible singularity coming from $K$ and the regularity of $W$. On a heuristic level, if the kernel $K(t, s)$ is behaving locally like $(t-s)^{-\eta}$ for $t \sim s$, and the Gaussian process has Hölder continuous trajectories of order $\alpha \in(0,1)$, then

$$
|K(t, s)(W(t)-W(s))|_{H} \lesssim(t-s)^{\alpha-\eta},
$$

and henceforth this composition is only finite for $t \rightarrow s$ whenever $\alpha-\eta>0$. Recalling that both in the classical probabilistic framework and in the modern approach of rough path theory, one would construct the integral as the limit when the mesh size of the partition $\mathcal{P}$ of $[0, t]$ goes to zero in the Riemann-type sum (in either $L^{2}(\Omega)$ if possible, or pathwise topology induced by variation or Hölder norms, see e.g. [18])

$$
\sum_{[u, v] \in \mathcal{P}} K(t, u)(W(v)-W(u)) .
$$


Thus at first glance, in order for this sum to converge, it seems natural to require $\alpha-\eta>0$ to (at least) avoid any explosions when the mesh of the partition $\mathcal{P}$ goes to 0 .

We next extend the Volterra Sewing Lemma to the two dimensional case, in order to construct two dimensional operator-valued Volterra integrals on the form

$$
\bar{Q}:=\int_{0}^{T} \int_{0}^{T^{\prime}} K(T, r) d^{2} Q\left(r, r^{\prime}\right) K\left(T^{\prime}, r^{\prime}\right)^{*}
$$

for linear operators $K$ and $Q$ on the Hilbert space $H$, and where $(s, t) \mapsto K(t, s)$ is possibly singular on the diagonal as described above. In this expression, $K^{*}$ is the adjoint operator of $K$, and the ordering of the integral appears naturally when considering operator-valued integrands which might be non-commutative. Our construction is based on Young-type integration theory with Volterra kernels, and only requires that $Q$ is Hölder regular, and $K$ does not blow up too fast at its singular point(s). In particular, we do not assume that $Q$ needs to be differentiable nor of bounded variation, and thus our construction truly extends the notion of the integral given in (1.2). An immediate consequence of our construction is stability of the two dimensional Volterra integral with respect to changes in the Volterra operator $K$ and the operator $Q$.

Through a consideration of the characteristic functional associated to the Volterra processes (1.1), we next show that when $Q=Q_{W}$ is of sufficient regularity, then the covariance operator $Q_{X}$ associated the Volterra process $X$ from (1.1) is given by $\bar{Q}$ in (1.5). In the end we discuss several application areas of our results, including an analysis of the covariance structure arising from general Gaussian Volterra iterated processes, the construction of the rough path associated to Volterra processes driven by Gaussian processes with irregular covariance structures, as well as a representation of the covariance structure of certain linear fractional stochastic differential equations of Ornstein-Uhlenbeck type in Hilbert space. In the last example, we discuss the potential application towards rough volatility modelling, proposing an extension of the rough Heston model to infinite dimensions.

Already in 2002, Towghi [32] proved that for two functions $f, g:[0, T]^{2} \rightarrow \mathbb{R}$, the following integral

$$
\int_{[0, T]^{2}} f(s, t) d Q_{W}(s, t)
$$

makes sense as the limit of a two dimensional Riemann type sum under suitable assumptions of complementary regularity between $f$ and $g$, and can thus be seen as an extension of the classical Young integral developed in [35]. The construction of the integral (1.5) can therefore be seen as an infinite dimensional extension of (1.6) to the case when the the integrand is given as a Volterra operator of singular type. In the case when the covariance $Q_{W}$ itself is a real-valued covariance function associated to a Volterra process, Lim provided in [20] a relaxation of the complementary regularity conditions originally proposed in [32] for existence of the two dimensional integral in (1.6). There, $f$ is assumed to be a sufficiently regular function, and thus singular Volterra kernels as we consider here fall outside of the scope of that article. Furthermore, in the current article, we do not impose any further structure on the covariance operator, other than regularity to keep it as general as possible, which in the end will prove useful in applications.

\subsection{Outline of the article}

The article is structured into the following sections:

Sec. 2 We give an introductory account of Gaussian processes in Hilbert space, as well as continuity of the trajectories. 
Sec. 3 We introduce the concept of Volterra paths (as described in [18]), and provide a pathwise construction of Gaussian Volterra paths from the regularity of the trajectories of the Gaussian noise as well as the possible singularity of the Volterra kernel.

Sec. 4 This section is devoted to prove that the pathwise Volterra processes driven by Gaussian processes, are again Gaussian. To this end, we show the construction of a general covariance operator, even when the covariance function of the driving noise is nowhere differentiable.

Sec. 5 We discuss a series of applications, including the construction of a rough path above Volterra processes driven by Gaussian noise with irregular covariance structures, and compute explicitly the covariance operators to various well-known Volterra processes, driven by Gaussian noise.

We provide background material on fractional calculus and some proofs of auxiliary results in two appendices.

\subsection{Notation}

We assume $(\Omega, \mathcal{F}, \mathbb{P})$ to be a complete probability space equipped with a filtration $\left(\mathcal{F}_{t}\right)_{t \geq 0}$ satisfying the usual hypotheses. We will work with a separable Hilbert space which will be denoted by $H$. The inner product in $H$ is denoted $\langle\cdot, \cdot\rangle_{H}$ with associated norm $|\cdot|_{H}$. The (Banach) space of bounded linear operators from $H$ to $E, E$ being another Hilbert space, is denoted $\mathcal{L}(H, E)$, with $\mathcal{L}(H):=\mathcal{L}(H, H)$. Sometimes $E$ may also be a general Banach space, but this will be clear from the context. We will frequently use the $n$-simplex $\Delta_{n}^{T}$ over an interval $[0, T]$ defined by

$$
\Delta_{n}^{T}:=\left\{\left(s_{1}, \ldots, s_{n}\right) \in[0, T]^{n} \mid s_{1} \geq \ldots \geq s_{n}\right\} .
$$

Also, define the diagonal in $[0, T]^{n}$ by $\mathrm{D}_{n}^{T}$, i.e.

$$
\mathrm{D}_{n}^{T}:=\left\{\left(s_{1}, \ldots, s_{n}\right) \in[0, T] \mid s_{1}=\ldots=s_{n}\right\} .
$$

We will denote by $\mathcal{C}^{\gamma}([0, T], H)$ the space of $\gamma$-Hölder continuous functions $f:[0, T] \rightarrow H$, with the norm $\|f\|_{\mathcal{C}^{\gamma}}=|f(0)|_{H}+\|f\|_{\gamma,[0, T]}$ where

$$
\|f\|_{\gamma,[0, T]}=\sup _{(t, s) \in \Delta_{2}^{T}} \frac{|f(t)-f(s)|_{H}}{|t-s|^{\gamma}} .
$$

Whenever the interval $[0, T]$ is clear from the context, we will write $\|f\|_{\gamma}$ for the quantity $\|f\|_{\gamma,[0, T]}$. Aiming towards an analysis of possibly non-smooth (i.e., only Hölder continuous) covariance functions, we will also be working with increments of two-parameter functions. To this end, we will need to introduce some new notation. Consider two points $s=\left(s_{1}, s_{2}\right)$ and $t=\left(t_{1}, t_{2}\right)$ in $[0, T]^{2}$ and a function $f:[0, T]^{2} \rightarrow H$. Let us denote by $\square_{s, t} f$ the generalized (or rectangular) increment of $f$ over the rectangle $[s, t]=\left[s_{1}, t_{1}\right] \times\left[s_{2}, t_{2}\right] \subset[0, T]^{2}$ (notice the implied partial order of the variables in $s=\left(s_{1}, s_{2}\right)$ and $\left.t=\left(t_{1}, t_{2}\right)\right)$ given by

$$
\square_{s, t} f=f\left(t_{1}, t_{2}\right)-f\left(t_{1}, s_{2}\right)-f\left(s_{1}, t_{2}\right)+f\left(s_{1}, s_{2}\right) .
$$

Note in particular that if $f$ has a mixed partial derivative $\partial^{2} f\left(r_{1}, r_{2}\right) / \partial r_{1} \partial r_{2}$ which is integrable over the rectangle $[s, t]$, we have

$$
\square_{s, t} f=\int_{s_{1}}^{t_{1}} \int_{s_{2}}^{t_{2}} \frac{\partial^{2} f\left(r_{1}, r_{2}\right)}{\partial r_{1} \partial r_{2}} d r_{2} d r_{1} .
$$

We remark in passing that in the literature $\square_{s, t} f$ is sometimes referred to as the $f$-volume of the rectangle $[s, t]$. 


\section{Gaussian stochastic processes in Hilbert spaces}

One of the main objectives in this article is to study the regularity properties of various stochastic processes in Hilbert spaces, together with their covariance operators. In this Section we provide some background material on the important class of Gaussian stochastic processes in Hilbert space which will be at the core of our studies.

For a Gaussian process in Hilbert space, one associates a covariance operator on the Hilbert space where the process lives, which describes the covariance structure of the process. A special case of such Gaussian processes is the $Q$-Wiener process, where the covariance operator $Q$ is a non-negative definite trace class linear operator. This process can be seen as an infinite dimensional extension of the well known Brownian motion, as these processes share many of the same probabilistic and analytic properties. The infinite dimensional Wiener process is a special case of a more general class of Hilbert-valued Gaussian stochastic processes. Below we give a general definition of Hilbert-valued Gaussian random variables, and then extend this definition to Hilbert-valued Gaussian processes. We highlight this definition with the example of the construction of the Hilbert-valued fractional Brownian motion.

We say that an $H$-valued random variable $X$ is square-integrable if $\mathbb{E}\left[|X|_{H}^{2}\right]<\infty$. If $X$ is square-integrable with zero mean, that is, $\mathbb{E}[X]=0$ where $0 \in H$ is the zero element and the expectation is in the sense of Bochner integration with respect to the probability $\mathbb{P}$, we introduce the covariance functional $Q$ associated to $X$ by

$$
Q=\mathbb{E}[X \otimes X] .
$$

Here, $\otimes$ is the tensor product such that for any $g, h, x \in H,(g \otimes h)(x)=\langle g, x\rangle_{H} h$. Note that by square-integrability of $X$, the expectation defining $Q$ is well-defined as a Bochner integral. It is known that $Q \in \mathcal{L}(H)$ is a symmetric, positive semi-definite trace class operator. In fact, we have, $\operatorname{Tr}(Q)=\mathbb{E}\left[|X|_{H}^{2}\right]$ and

$$
\mathbb{E}\left[\langle X, g\rangle_{H}\langle X, h\rangle_{H}\right]=\langle Q g, h\rangle_{H},
$$

for any $g, h \in H$. We have the following standard definition of a Gaussian random variable in Hilbert space:

Definition 1. An $H$-valued random variable $X$ is said to be Gaussian if $\langle X, h\rangle_{H}$ is a real-valued Gaussian random variable for every $h \in H$.

We remark that Gaussian variables in Hilbert space are square-integrable (see [26, Thm. 3.31]). We introduce a Gaussian process in Hilbert space by the following definition (see [26, Def. 3.30]):

Definition 2. An $H$-valued stochastic process $(X(t))_{t \geq 0}$ is said to be Gaussian if for every $n \in \mathbb{N}, 0 \leq t_{1}<t_{2} \cdots<t_{n}<\infty,\left(X\left(t_{1}\right), X\left(t_{2}\right), \ldots, X\left(t_{n}\right)\right)$ is an $H^{n}$-valued Gaussian random variable.

By definition, we have that a Gaussian process can be equivalently characterised by saying that for every $n \in \mathbb{N}, 0 \leq t_{1}<t_{2} \cdots<t_{n}<\infty$ and $h_{1}, \ldots, h_{n} \in H$, $\left(\left\langle X\left(t_{1}\right), h_{1}\right\rangle_{H}, \ldots,\left\langle X\left(t_{n}\right), h_{n}\right\rangle_{H}\right)$ is an $n$-variate Gaussian random variable on $\mathbb{R}^{n}$. We have a covariance operator defined as (for $s, t \geq 0$ )

$$
Q(s, t):=\mathbb{E}[X(s) \otimes X(t)] \in \mathcal{L}(H) .
$$

Here we have implicitly assumed that the process has zero mean. Note that generally $Q(s, t) \neq Q(t, s)$. But,

$$
\langle Q(s, t) g, h\rangle_{H}=\mathbb{E}\left[\langle X(s), g\rangle_{H}\langle X(t), h\rangle_{H}\right]=\langle g, Q(t, s) f\rangle_{H},
$$


and thus, $Q(s, t)^{*}=Q(t, s)$. But, on the other hand, $Q(t, t)$ is a positive semi-definite and symmetric trace class operator.

An important Gaussian process in Hilbert space is the $Q$-Wiener process, which has a covariance operator $Q(s, t)=Q \min (s, t)$ where $Q$ is a symmetric positive definite trace class operator. As in $[31,12,16]$ we can define a $Q$-fractional Brownian motion with values in Hilbert space by letting

$$
Q(s, t):=R^{h}(s, t) Q,
$$

for a symmetric positive definite trace class operator $Q$ and the real-valued function

$$
R^{h}(s, t)=\frac{1}{2}\left(s^{2 h}+t^{2 h}-|t-s|^{2 h}\right),
$$

with the Hurst index $h \in(0,1)$ and $s, t \geq 0$. Letting $h=0.5$, the $Q$-fractional Brownian motion is a $Q$-Wiener process.

In our analysis, the continuity properties of paths play an important role. For this purpose, we recall the Kolmogorov continuity theorem (see e.g. [11, Thm. 3.3], where a full proof of the below statement can be found).

Theorem 3. (Kolmogorov's continuity theorem). Let $W: \Omega \times[0, T] \rightarrow H$ be a stochastic process such that for some positive constants $C>0, \epsilon>0, \delta>1$ and all $(t, s) \in \Delta_{2}^{T}$ the following inequality holds

$$
\mathbb{E}\left[|W(t)-W(s)|_{H}^{\delta}\right] \leq C|t-s|^{1+\epsilon} .
$$

Then there exists a pathwise continuous modification $\widetilde{W}$ of $W$. More specifically, the mapping $t \mapsto \widetilde{W}(\omega, t)$ is $\alpha$-Hölder continuous with $\alpha=\frac{\epsilon}{\delta}, \mathbb{P}-$ a.s.

For a $Q$-Wiener process, we readily see that

$$
\mathbb{E}\left[|W(t)-W(s)|_{H}^{2}\right]=|t-s| \operatorname{Tr}(Q),
$$

while for the fractional Brownian motion with covariance operator defined in (2.1) we have

$$
\mathbb{E}\left[|W(t)-W(s)|_{H}^{2}\right]=|t-s|^{2 h} \operatorname{Tr}(Q) .
$$

We have the following result on the Hölder continuity of the fractional Brownian motion (which seems to be known but we include a proof for the convenience of the reader):

Proposition 4. Let $W$ be a $Q$-fractional Brownian motion with values in $H$ and covariance operator given in (2.1) with Hurst parameter $h \in(0,1)$. Then, for $(t, s) \in \Delta_{2}^{T}$

$$
\mathbb{E}\left[|W(t)-W(s)|_{H}^{2 n}\right] \leq|t-s|^{2 h n}(\operatorname{Tr}(Q))^{n} \mathbb{E}\left[Z^{2 n}\right]
$$

for any $n \in \mathbb{N}$ and with $Z$ being a standard normal random variable in $\mathbb{R}$. Moreover, there exists a version of $W$ which is Hölder continuous of order $\alpha<h, \mathbb{P}-$ a.s.

Proof. Let $\left(e_{i}\right)_{i \in \mathbb{N}}$ be the ONB of eigenvectors of $Q$, with the covariance operator $Q(s, t)$ of $W$ defined in (2.1). We have that $W(t)-W(s)$ is a Gaussian mean-zero random variable, and a straightforward calculation yields that it has the covariance operator $|t-s|^{2 h} Q$. Thus, $X_{i}:=\left\langle W(t)-W(s), e_{i}\right\rangle_{H}$ is a mean-zero real-valued Gaussian random variable, with variance equal to $|t-s|^{2 h} \lambda_{i}$. Here, $\lambda_{i}>0$ is the $i$ th eigenvalue of $Q$. As $\left(e_{i}\right)_{i \in \mathbb{N}}$ are the eigenvectors of $Q, X_{i}$ is independent of $X_{j}$ for any $i \neq j, i . j \in \mathbb{N}$. Let $\left(Z_{i}\right)_{i \in \mathbb{N}}$ be a sequence of independent identically distributed real valued standard normal variables. Then, in distribution, we have $X_{i}=|t-s|^{h} \sqrt{\lambda_{i}} Z_{i}$. Parseval's equality yields

$$
\mathbb{E}\left[|W(t)-W(s)|_{H}^{2 n}\right]=\mathbb{E}\left[\left(\sum_{i=1}^{\infty}\left\langle W(t)-W(s), e_{i}\right\rangle_{H}^{2}\right)^{n}\right]
$$




$$
=|t-s|^{2 h n} \mathbb{E}\left[\left(\sum_{i=1}^{\infty} \lambda_{i} Z_{i}^{2}\right)^{n}\right]
$$

If $n=1$, we are done. Suppose that $n \geq 2$. For $p>1$ and $q$ being the reciprocal of $p$, we find by Hölder's inequality

$$
\begin{aligned}
\sum_{i=1}^{\infty} \lambda_{i} Z_{i}^{2} & =\sum_{i=1}^{\infty} \lambda_{i}^{1 / q} \lambda_{i}^{1 / p} Z_{i}^{2} \\
& \leq\left(\sum_{i=1}^{\infty} \lambda_{i}\right)^{1 / q}\left(\sum_{i=1}^{\infty} \lambda_{i} Z_{i}^{2 p}\right)^{1 / p} \\
& =(\operatorname{Tr}(Q))^{1 / q}\left(\sum_{i=1}^{\infty} \lambda_{i} Z_{i}^{2 p}\right)^{1 / p}
\end{aligned}
$$

Choosing $p=n>1$ and $q=n / n-1$, we find

$$
\mathbb{E}\left[|W(t)-W(s)|_{H}^{2 n}\right] \leq|t-s|^{2 h n}(\operatorname{Tr}(Q))^{n-1} \sum_{i=1}^{\infty} \lambda_{i} \mathbb{E}\left[Z_{i}^{2 n}\right],
$$

and the first result of the Proposition follows.

For the second conclusion, suppose that $n \in \mathbb{N}$ is such that $2 h n>1$. Then we obtain existence of an $\alpha:=h-\frac{1}{2 n}$ Hölder continuous version of $W$ from Kolmogorov's continuity theorem 3. As $n$ can be chosen arbitrary large, we conclude that there exists a version of $W$ which is Hölder continuous of order $\alpha<h$, P-a.s.

As a simple consequence of the above, we see that a $Q$-Wiener process has a version with Hölder continuous paths of order $\alpha<1 / 2$. In the analysis that follows in the next sections, we will make use of processes with specific regularity properties of the paths. The discussion in this Section shows that we have available specific cases of (Gaussian) stochastic processes with various Hölder regularity of the paths. Gaussian processes will constitute our canonical class of models, and whenever we refer to such processes we will have their Hölder continuous version in mind.

\section{Pathwise Volterra processes in Hilbert spaces}

In this Section we introduce and study Volterra processes of the form (1.1). In order to give a pathwise description of Volterra integrals driven by generic Hölder paths, we will apply a variant of the celebrated Sewing Lemma from the theory of rough paths, modified to accommodate the Volterra structure inherit in the processes of interest. This lemma was first proved in [18] where the authors extend aspects of the theory of rough paths to the analysis of Volterra equations with singular kernels driven by irregular paths. In order to discuss Volterra integration in a pathwise manner, we will introduce an abstract space of Volterra paths, as defined in [18]. This definition allows us to discuss the continuity properties of Volterra paths, independent of the Volterra integral representation. However, it will be instructive for the reader to think of the expression

$$
X^{\tau}(t):=X(\tau, t)=\int_{0}^{t} K(\tau, r) d W(r),
$$

where we have chosen to let a Volterra process have two arguments by decoupling the first argument $\tau$ in the kernel, and the upper integration parameter $t$, with $\tau \geq t$. The classical Volterra process is of course given by the mapping $t \mapsto X^{t}(t)$ (recall (1.1)). 
Thus, if $W:[0, T] \rightarrow H$ is a smooth path, ${ }^{1}$ then the integral in (3.1) can be interpreted in the Riemann sense, provided that the kernel $K \in \mathcal{L}(H)$ is Riemann integrable with respect to $W$, and thus we can view $X$ as a path from $\Delta_{2}^{T}$ to $H$. Note that we can then measure the regularity of $X$ in both $t$ and $\tau$ separately, where at least at a heuristic level, the regularity of $X$ in the $\tau$ parameter can be expected to be inherited from the regularity of the kernel $K$ in $\tau$. On the other hand the regularity of $X$ in the $t$ parameter will typically be inherited by the path $W$.

Definition 5. Let $\gamma, \eta \in(0,1)$ and assume $\gamma-\eta>0$. We denote by $\mathcal{V}^{(\gamma, \eta)}\left(\Delta_{2}^{T}, H\right)$ the space of all functions $f: \Delta_{2}^{T} \rightarrow H$ such that

$$
\|f\|_{(\gamma, \eta)}:=\|f\|_{(\gamma, \eta), 1}+\|f\|_{(\gamma, \eta), 1,2}<\infty
$$

where we define the semi-norms by

$$
\begin{aligned}
& \|f\|_{(\gamma, \eta), 1}:=\sup _{(\tau, t, s) \in \Delta_{3}^{T}} \frac{\left|f^{\tau}(t)-f^{\tau}(s)\right|_{H}}{\left[|\tau-t|^{-\eta}|t-s|^{\gamma}\right] \wedge|\tau-s|^{\gamma-\eta}} \\
& \|f\|_{(\gamma, \eta), 1,2}:=\sup _{\substack{\left(\tau^{\prime}, \tau, t, s\right) \in \Delta_{4}^{T} \\
\theta \in[0,1], \zeta \in[0, \gamma-\eta)}} \frac{\left|f^{\tau^{\prime}}(t)-f^{\tau}(t)-f^{\tau^{\prime}}(s)+f^{\tau}(s)\right|_{H}}{\left|\tau^{\prime}-\tau\right|^{\theta}|\tau-t|^{-\theta+\zeta}\left\{\left[|\tau-t|^{-\eta-\zeta}|t-s|^{\gamma}\right] \wedge|\tau-s|^{\gamma-\eta-\zeta\}}\right.} .
\end{aligned}
$$

Here we have used the notation $f^{\tau}(t):=f(\tau, t)$ for $(\tau, t) \in \Delta_{2}^{T}$.

Remark 6. Consider a subspace $\hat{\mathcal{V}}^{(\gamma, \eta)}\left(\Delta_{2}^{T}, H\right) \subset \mathcal{V}^{(\gamma, \eta)}\left(\Delta_{2}^{T}, H\right)$ containing all Volterra paths $f \in \mathcal{V}^{(\gamma, \eta)}\left(\Delta_{2}^{T}, H\right)$ such that $f_{0}:=f^{\tau}(0)=c \in H$ for all $\tau \in[0, T]$. Under the norm

$$
\|f\|_{(\gamma, \eta), *}:=\left|f_{0}\right|_{H}+\|f\|_{(\gamma, \eta)}
$$

the space $\hat{\mathcal{V}}^{(\gamma, \eta)}\left(\Delta_{2}^{T}, H\right)$ is a Banach space, see e.g. [18].

Remark 7. We can extend the definition of $\mathcal{V}^{(\gamma, \eta)}\left(\Delta_{2}^{T}, H\right)$ above to functions $f: \Delta_{3}^{T} \rightarrow H$, where $f$ has one upper variable and two lower variables, that is,

$$
(\tau, t, s) \mapsto f^{\tau}(t, s)
$$

In this case, we consider the semi-norms $\|f\|_{(\gamma, \eta), 1}$ and $\|f\|_{(\gamma, \eta), 1,2}$ to be given by

$$
\begin{aligned}
& \|f\|_{(\gamma, \eta), 1}:=\sup _{(\tau, t, s) \in \Delta_{3}^{T}} \frac{\left|f^{\tau}(t, s)\right|_{H}}{|\tau-t|^{-\eta}|t-s|^{\gamma} \wedge|\tau-s|^{\gamma-\eta}} \\
& \|f\|_{(\gamma, \eta), 1,2}:=\sup _{\substack{\left(\tau^{\prime}, \tau, t, s\right) \in \Delta_{4}^{T} \\
\theta \in[0,1], \zeta \in[0, \gamma-\eta)}} \frac{\left|f^{\tau^{\prime}}(t, s)-f^{\tau}(t, s)\right|_{H}}{\left|\tau^{\prime}-\tau\right|^{\theta}|\tau-t|^{-\theta+\zeta}\left[|\tau-t|^{-\eta-\zeta}|t-s|^{\gamma} \wedge|\tau-s|^{\gamma-\eta-\zeta]}\right.} .
\end{aligned}
$$

We denote the space of such three-variable functions by $\mathcal{V}_{3}^{(\gamma, \eta)}\left(\Delta_{3}^{T}, H\right)$.

The next proposition shows the relation between the space of classical Hölder paths $\mathcal{C}^{\rho}([0, T], H)$ and $\mathcal{V}^{(\gamma, \eta)}\left(\Delta_{2}^{T}, H\right)$ when $\gamma-\eta=\rho>0$.

Proposition 8. Suppose $f \in \mathcal{V}^{(\gamma, \eta)}\left(\Delta_{2}^{T}, H\right)$ with $\gamma-\eta=\rho>0$ and $f^{\tau}(0)=c \in H$ is constant (in $H$ ) for all $\tau \in[0, T]$. Then the restriction of $\tilde{f}(t):=f^{t}(t)$ of $f$ to the diagonal of $\Delta_{2}^{T}$ is $\zeta$-Hölder continuous for any $\zeta \in[0, \rho)$, i.e. $\tilde{f} \in \mathcal{C}^{\zeta}([0, T], H)$.

\footnotetext{
${ }^{1}$ Notice here that $W$ is a general path, not necessarily a Gaussian process as we discussed in the previous section. However, in typical cases we have $W$ being a $Q$-Wiener or fractional Brownian motion, which explains why we use the notation $W$.
} 
Proof. By assumption it follows that $f^{t}(0)-f^{s}(0)=0$ for all $s, t \in[0, T]$. Furthermore, by definition of the norms, we have that

$$
\begin{aligned}
\left|f^{t}(t)-f^{s}(s)\right|_{H} & \leq\left|f^{t}(t)-f^{t}(s)\right|_{H}+\left|f^{t}(s)-f^{s}(s)\right|_{H} \\
& \leq\left|f^{t}(t)-f^{t}(s)\right|_{H}+\left|f^{t}(s)-f^{s}(s)-f^{t}(0)+f^{s}(0)\right|_{H} \\
& \leq\|f\|_{(\gamma, \eta), 1}|t-s|^{\rho}+T^{\gamma-\eta-\zeta}\|f\|_{(\gamma, \eta), 1,2}|t-s|^{\zeta} .
\end{aligned}
$$

In the second majorization of the last inequality above, we applied the definition of $\|\cdot\|_{(\gamma, \eta), 1,2}$ in (3.2) with $\theta=\zeta \in[0, \gamma-\eta)$, i.e., for any $\left(\tau^{\prime}, \tau, t, s\right) \in \Delta_{4}^{T}$, the following relation holds

$$
\left|f^{\tau^{\prime}}(t)-f^{\tau}(t)-f^{\tau^{\prime}}(s)+f^{\tau}(s)\right|_{H} \leq\|f\|_{(\gamma, \eta), 1,2}\left|\tau^{\prime}-\tau\right|^{\zeta}|\tau-t|^{0}|\tau-s|^{\gamma-\eta-\zeta} .
$$

Thus, for $\left(\tau^{\prime}, \tau, t, s\right):=(t, s, s, 0)$ we get the desired inequality after observing that $s^{\gamma-\eta-\zeta} \leq T^{\gamma-\eta-\zeta}$. As $\zeta \in[0, \rho)$ is arbitrary, we see that $\tilde{f} \in \mathcal{C}^{\zeta}([0, T], H)$ and the result follows.

In order to accommodate pathwise Volterra integrals, we will need a modified version of the Sewing Lemma. But first we define a suitable space of abstract Volterra integrands. In the sequel, we will work with integrals taking values in a space of linear operators on Hilbert spaces. We therefore state the Volterra Sewing Lemma in general Banach spaces.

Definition 9. Consider a Banach space $E$, and suppose $\gamma, \eta \in(0,1), \beta \in(1, \infty)$ and $\kappa \in(0,1)$ is such that the following relations hold $\beta-\kappa \geq \gamma-\eta>0$. Denote by $\mathscr{V}^{(\gamma, \eta)(\beta, \kappa)}\left(\Delta_{3}^{T}, E\right)$, the space of all functions $\Xi: \Delta_{3}^{T} \rightarrow E$ such that

$$
\|\Xi\|_{(\gamma, \eta)(\beta, \kappa)}:=\|\Xi\|_{(\gamma, \eta)}+\|\delta \Xi\|_{(\beta, \kappa)}<\infty .
$$

Here $\delta$ is the operator defined for any $s \leq u \leq t \leq \tau$ acting on functions $g$ by

$$
\delta_{u} g^{\tau}(t, s)=g^{\tau}(t, s)-g^{\tau}(t, u)-g^{\tau}(u, s) .
$$

The norm $\|\Xi\|_{(\gamma, \eta)}$ is given as in Remark 7, while the quantity $\|\delta \Xi\|_{(\beta, \kappa)}$ is a slight modification of the norms from Remark 7 defined by

$$
\|\delta \Xi\|_{(\beta, \kappa)}:=\|\delta \Xi\|_{(\beta, \kappa), 1}+\|\delta \Xi\|_{(\beta, \kappa), 1,2}
$$

where

$$
\begin{gathered}
\|\delta \Xi\|_{(\beta, \kappa), 1}:=\sup _{(\tau, t, u, s) \in \Delta_{4}^{T}} \frac{\left|\delta_{u} \Xi^{\tau}(t, s)\right|_{E}}{|\tau-t|^{-\kappa}|t-s|^{\beta} \wedge|t-s|^{\beta-\kappa}}, \\
\|\delta \Xi\|_{(\beta, \kappa), 1,2}:=\sup _{\substack{\left(\tau^{\prime}, \tau, t, u, s\right) \in \Delta_{5}^{T} \\
\theta \in[0,1], \zeta \in[0, \beta-\kappa)}} \frac{\left|\delta_{u}\left[\Xi^{\tau^{\prime}}(t, s)-\Xi^{\tau}(t, s)\right]\right|_{E}}{\left|\tau^{\prime}-\tau\right|^{\theta}|\tau-t|^{-\theta+\zeta}\left[|\tau-u|^{\left.-\kappa-\zeta|t-s|^{\beta}\right]}\right.} .
\end{gathered}
$$

where we mean $\Xi^{\tau}(t, s):=\Xi(\tau, t, s)$. In the sequel we call $\mathscr{V}(\gamma, \eta)(\beta, \kappa)\left(\Delta_{3}^{T}, E\right)$ the space of all abstract Volterra integrands.

We are now ready to state the Sewing Lemma adapted to Volterra integrands. The following lemma is a trivial extension of [18, Lemma 21] to the case of Banach-valued Volterra kernels.

Lemma 10. (Volterra Sewing Lemma) Let $E$ be a Banach space, and consider parameters $\gamma, \eta \in(0,1), \beta \in(1, \infty)$, and $\kappa \in(0,1)$ such that $\beta-\kappa \geq \gamma-\eta>0$. There exists a unique continuous map $\mathcal{I}: \mathscr{V}^{(\gamma, \eta)(\beta, \kappa)}\left(\Delta_{3}^{T}, E\right) \rightarrow \mathcal{V}^{(\gamma, \eta)}\left(\Delta_{2}^{T}, E\right)$ such that the following statements holds true 
(i). The quantity $\mathcal{I}\left(\Xi^{\tau}\right)(t, s):=\lim _{|\mathcal{P}| \rightarrow 0} \sum_{[u, v] \in \mathcal{P}} \Xi^{\tau}(v, u)$ exists (in $E$ ) for all tuples $(\tau, t, s) \in \Delta_{3}^{T}$, where $\mathcal{P}$ is a generic partition of $[s, t]$ and $|\mathcal{P}|$ denotes the mesh size of the partition.

(ii). For all $(\tau, t, s) \in \Delta_{3}^{T}$ the following inequality holds

$$
\left|\mathcal{I}\left(\Xi^{\tau}\right)(t, s)-\Xi^{\tau}(t, s)\right|_{E} \lesssim \mid\|\delta \Xi\|_{(\beta, \kappa), 1}\left(|\tau-t|^{-\kappa}|t-s|^{\beta} \wedge|\tau-s|^{\beta-\kappa}\right),
$$

(iii). For all $\left(\tau^{\prime}, \tau, t, s\right) \in \Delta_{4}^{T}, \theta \in[0,1]$ and $\zeta \in[0, \beta-\kappa)$, we denote by $\Xi^{\tau^{\prime}, \tau}(t, s)=$ $\Xi^{\tau^{\prime}}(t, s)-\Xi^{\tau}(t, s)$, and the following inequality holds

$$
\begin{aligned}
& \left|\mathcal{I}\left(\Xi^{\tau^{\prime}, \tau}\right)(t, s)-\Xi^{\tau^{\prime}, \tau}(t, s)\right|_{E} \\
& \quad \lesssim\|\mid \delta \Xi\|_{(\beta, \kappa), 1,2}\left(\left|\tau^{\prime}-\tau\right|^{\theta}|\tau-t|^{-\theta+\zeta}\left[|\tau-t|^{-\kappa-\zeta}|t-s|^{\beta} \wedge|\tau-s|^{\beta-\kappa-\zeta}\right]\right) .
\end{aligned}
$$

Moreover, $t \mapsto \mathcal{I}\left(\Xi^{\tau}\right)(t):=\mathcal{I}\left(\Xi^{\tau}\right)(t, 0)$ is additive, in the sense that $\mathcal{I}\left(\Xi^{\tau}\right)(t, s)=$ $\mathcal{I}\left(\Xi^{\tau}\right)(t, 0)-\mathcal{I}\left(\Xi^{\tau}\right)(s, 0)$, and we conclude that $(\tau, t) \mapsto \mathcal{I}\left(\Xi^{\tau}\right)(t) \in \mathcal{V}^{(\gamma, \eta)}\left(\Delta_{2}^{T}, E\right)$.

We are frequently going to work with Volterra kernels in various contexts, and we therefore state a common hypothesis on the regularity on the kernels we consider in this article.

Definition 11. For $\eta \in(0,1)$, suppose $K: \Delta_{2}^{T} \rightarrow \mathcal{L}(H)$ is a linear operator on the Hilbert space $H$ which satisfies for $(\tau, t, s, r) \in \Delta_{4}^{T}$ and any $\theta, \nu \in[0,1]$ the following inequalities

$$
\begin{aligned}
|K(t, s) f|_{H} & \lesssim|t-s|^{-\eta}|f|_{H} \\
|(K(t, s)-K(t, r)) f|_{H} & \lesssim|t-s|^{-\eta-\theta}|s-r|^{\theta}|f|_{H} . \\
|(K(\tau, s)-K(t, s)) f|_{H} & \lesssim|t-s|^{-\eta-\theta}|\tau-t|^{\theta}|f|_{H} . \\
|(K(\tau, s)-K(\tau, r)-K(t, s)+K(t, r)) f|_{H} & \lesssim|\tau-r|^{-\nu-\theta-\eta}|\tau-t|^{\theta}|r-s|^{\nu}|f|_{H} .
\end{aligned}
$$

for every $f \in H$. Then we say that the kernel $K$ is a Volterra kernel of order $\eta$. We denote the space of all Volterra kernels $K$ of order $\eta \in(0,1)$ satisfying (3.8)-(3.11) by $\mathcal{K}_{\eta}$. We equip this space with the following semi-norm

$$
\|K\|_{\mathcal{K}_{\eta}}:=\|K\|_{\eta, 1}+\|K\|_{\eta, 2}+\|K\|_{\eta, 3},\|K\|_{\eta, 4},
$$

where we define the three semi-norms on the right-hand side above by

$$
\begin{aligned}
& \|K\|_{\eta, 1}:=\sup _{(t, s) \in \Delta_{2}^{T}} \frac{\|K(t, s)\|_{\mathrm{op}}}{|t-s|^{-\eta}} \\
& \|K\|_{\eta, 2}:=\sup _{\substack{(t, u, s) \in \Delta_{3}^{T} \\
\theta \in[0,1]}} \frac{\|K(t, s)-K(u, s)\|_{\mathrm{op}}}{|t-u|^{\theta}|u-s|^{-\theta-\eta}} \\
& \|K\|_{\eta, 3}:=\sup _{\substack{(t, u, s) \in \Delta_{3}^{T} \\
\theta \in[0,1]}} \frac{\|K(t, u)-K(t, s)\|_{\mathrm{op}}}{|u-s|^{\theta}|t-u|^{-\theta-\eta}}, \\
& \|K\|_{\eta, 4}:=\sup _{\substack{\left(\tau^{\prime}, \tau, s, r\right) \in \Delta_{4}^{T} \\
\theta, \nu \in[0,1]}} \frac{\left\|K\left(\tau^{\prime}, s\right)-K\left(\tau^{\prime}, r\right)-K(\tau, s)+K(\tau, r)\right\|_{\mathrm{op}}}{|\tau-r|^{-\nu-\theta-\eta}\left|\tau^{\prime}-\tau\right|^{\nu}|r-s|^{\theta}},
\end{aligned}
$$

with $\|\cdot\|_{\text {op }}$ denoting the operator norm.

Remark 12. Note that if $K \in \mathcal{K}_{\eta}$, then also $K^{*} \in \mathcal{K}_{\eta}$. Indeed, this follows from the well-known fact that $\|K(t, s)\|_{\text {op }}=\left\|K^{*}(t, s)\right\|_{\text {op }}$ for any $(t, s) \in \Delta_{2}^{T}$. 
Remark 13. We restrict our analysis here to $K(t, s) \in \mathcal{L}(H)$. One could easily extend our results to operators $K(t, s) \in \mathcal{L}\left(H, H^{\prime}\right)$ for some general Hilbert spaces $H$ and $H^{\prime}$, or even to $K(t, s) \in \mathcal{L}\left(E, E^{\prime}\right)$ for some general Banach spaces $E$ and $E^{\prime}$, by adjusting the spaces of paths and functions accordingly. However, to increase readability we confine our considerations to $\mathcal{L}(H)$.

With Definition 11 at hand we will now show that we can construct Volterra processes from Hölder paths in a deterministic manner using the Volterra Sewing Lemma 10.

Proposition 14. Suppose $\gamma, \eta \in(0,1)$ are such that $\gamma-\eta>0$. Let $W \in \mathcal{C}^{\gamma}([0, T], H)$ and consider a kernel $K \in \mathcal{K}_{\eta}$ as introduced in Definition 11. Let the abstract integrand $\Xi$ be given as

$$
\Xi^{\tau}(t, s):=K(\tau, s)(W(t)-W(s)) .
$$

Then we define the pathwise Volterra process as the integral

$$
X^{\tau}(t):=\int_{0}^{t} K(\tau, s) d W(s):=\mathcal{I}\left(\Xi^{\tau}\right)(t),
$$

where, for a partition $\mathcal{P}$ of $[0, t]$, the integral $\mathcal{I}\left(\Xi^{\tau}\right)$ is defined as in Lemma 10 by

$$
\mathcal{I}\left(\Xi^{\tau}\right)(t):=\lim _{|\mathcal{P}| \rightarrow 0} \sum_{[u, v] \in \mathcal{P}} \Xi^{\tau}(v, u),
$$

and the limit is taken in $H$. Moreover, we have that $(\tau, t) \mapsto X^{\tau}(t) \in \mathcal{V}^{(\gamma, \eta)}\left(\Delta_{2}^{T}, H\right)$.

Proof. With Lemma 10 in mind, we recall that in order to show convergence of the integral in (3.19), it is sufficient to prove that $\Xi$ given as in (3.17) satisfies the following conditions

$$
\begin{aligned}
\|\Xi\|_{(\gamma, \eta)} & =\|\Xi\|_{(\gamma, \eta), 1}+\|\Xi\|_{(\gamma, \eta), 1,2}<\infty \quad \text { and } \\
\|\delta \Xi\|_{(\beta, \kappa)} & =\|\delta \Xi\|_{(\beta, \kappa), 1}+\|\delta \Xi\|_{(\beta, \kappa), 1,2}<\infty,
\end{aligned}
$$

for some $(\beta, \kappa) \in(1, \infty) \times[0,1)$ with $\beta-\kappa \geq \gamma-\eta$. The fact that $\|\Xi\|_{(\gamma, \eta)}<\infty$ follows directly from the assumptions on the noise $W$ and kernel $K$ : Indeed, since $K \in \mathcal{K}_{\eta}$ and $W \in \mathcal{C}^{\gamma}([0, T], H)$ yields

$$
|K(\tau, s)(W(t)-W(s))|_{H} \lesssim\|K\|_{\eta, 1}\|W\|_{\gamma}|\tau-s|^{-\eta}|t-s|^{\gamma} .
$$

Notice that since $\tau \geq t \geq s$ we have

$$
\left.|\tau-s|^{-\eta}|t-s|^{\gamma} \leq\left[|\tau-t|^{-\eta} \mid t-s\right]^{\gamma}\right] \wedge|\tau-s|^{\gamma-\eta} .
$$

This shows that $\|\Xi\|_{(\gamma, \eta), 1}<\infty$. For the second part of the semi-norm $\|\Xi\|_{(\gamma, \eta)}$, we argue as follows. Firstly, for $\tau^{\prime} \geq \tau$, we find

$$
\Xi^{\tau^{\prime}}(t, s)-\Xi^{\tau}(t, s)=\left(K\left(\tau^{\prime}, s\right)-K(\tau, s)\right)(W(t)-W(s))
$$

Hence, from the semi-norm in (3.15), we find

$$
\left|\left(K\left(\tau^{\prime}, s\right)-K(\tau, s)\right)(W(t)-W(s))\right|_{H} \leq\|W\|_{\gamma}\|K\|_{\eta, 3}\left|\tau^{\prime}-\tau\right|^{\theta}|\tau-s|^{-\theta-\eta}|t-s|^{\gamma} .
$$

for any $\theta \in[0,1]$. Invoking (3.20), it is readily seen that also $\|\Xi\|_{(\gamma, \eta), 1,2}<\infty$.

Next we move on with showing the finiteness of $\|\delta \Xi\|_{(\beta, \kappa)}$ : First, we investigate the action of $\delta$ on the integrand $\Xi$ given in (3.17). By elementary algebraic manipulations, we observe that for $(\tau, t, u, s) \in \Delta_{4}^{T}$ the following relation holds

$$
\delta_{u} \Xi^{\tau}(t, s)=(K(\tau, s)-K(\tau, u))(W(t)-W(u)) .
$$


Again using that the kernel $K \in \mathcal{K}_{\eta}$ and the assumption that $W \in \mathcal{C}^{\gamma}([0, T], H)$, it is readily checked that for any $\theta \in[0,1]$

$$
\left|\delta_{u} \Xi^{\tau}(t, s)\right|_{H} \leq\|K\|_{\eta, 3}\|W\|_{\gamma}|\tau-s|^{-\eta-\theta}|s-u|^{\theta}|t-u|^{\gamma} \lesssim|\tau-s|^{-\eta-\theta}|t-s|^{\gamma+\theta} .
$$

We therefore set $\beta=\gamma+\theta$ and $\kappa=\eta+\theta$, and choose $\theta \in[0,1]$ such that $(\beta, \kappa) \in$ $(1, \infty) \times(0,1)$ (we note that this is always possible due to the restriction $\gamma-\eta>0$ ). Then we see that $|\tau-s| \geq|t-s|$ and $|\tau-s| \geq|\tau-t|$, and therefore

$$
|\tau-s|^{-\kappa}|t-s|^{\beta} \leq\left[|\tau-t|^{-\kappa}|t-s|^{\beta}\right] \wedge|t-s|^{\beta-\kappa}
$$

It follows that $\|\delta \Xi\|_{(\beta, \kappa), 1}<\infty$. We also point out that $\beta-\kappa=\gamma-\eta$.

To prove that also $\|\delta \Xi\|_{(\beta, \kappa), 1,2}<\infty$, we follow in the same direction as outlined above. However, rather than invoking (3.9), we will need to make use of (3.11). In particular, we need to consider the increment in the upper variables in $\Xi$, i.e.

$$
\Xi^{\tau^{\prime}, \tau}(t, s)=\Xi^{\tau^{\prime}}(t, s)-\Xi^{\tau}(t, s),
$$

and then the action of $\delta_{u}$ on $\Xi^{\tau^{\prime}}, \tau(t, s)$ is given by

$$
\delta_{u} \Xi^{\tau^{\prime}, \tau}(t, s)=\left(K\left(\tau^{\prime}, s\right)-K(\tau, s)-K\left(\tau^{\prime}, u\right)+K(\tau, u)\right)(W(t)-W(u)) .
$$

Thus invoking (3.11) on the kernel $K$, and we can follow the exact same routine as for the proof that $\|\delta \Xi\|_{(\beta, \kappa), 1}<\infty$. One sees that for any parameters $\theta, \nu \in[0,1]$ and $\left(\tau^{\prime}, \tau, t, u, s\right) \in \Delta_{5}^{T}$ we have

$$
\left|\delta_{u} \Xi^{\tau^{\prime}, \tau}(t, s)\right|_{H} \leq\|K\|_{\eta, 4}\|W\|_{\gamma}\left|\tau^{\prime}-\tau\right|^{\nu}|\tau-u|^{-\nu-\theta-\eta}|u-s|^{\theta}|t-u|^{\gamma} .
$$

Using that for any $\zeta \geq 0$ we have $|\tau-u|^{-\nu-\theta-\eta} \leq|\tau-t|^{-\theta+\zeta}|\tau-u|^{-\eta-\nu-\zeta}$, we obtain that

$$
\left|\delta_{u} \Xi^{\tau^{\prime}, \tau}(t, s)\right|_{H} \leq\|K\|_{\eta, 4}\|W\|_{\gamma}\left|\tau^{\prime}-\tau\right|^{\nu}|\tau-t|^{-\nu+\zeta}\left[|\tau-u|^{-\eta-\theta-\zeta}|t-s|^{\gamma+\theta}\right]
$$

We then choose $\theta \in[0,1]$ such that $\gamma+\theta>1$ and $\theta+\eta+\zeta<1$, which is possible by restricting $\zeta \in[0, \gamma-\rho)$, and $\gamma-\rho>0$ by assumption. We therefore set $\beta=\theta+\gamma$ and $\kappa=\theta+\eta$, and it follows that $\|\delta \Xi\|_{(\beta, \kappa), 1,2}<\infty$, where we recall that this norm is defined in (3.5). Thus we may invoke Lemma 10 for the construction of the integral $\mathcal{I}(\Xi)$ as given in 3.19, and we get that this integral exists with a unique limit. It follows directly from Lemma 10 that $X \in \mathcal{V}^{(\gamma, \eta)}\left(\Delta_{2}^{T}, H\right)$. This concludes the proof.

Let us illustrate Proposition 14 by providing an example which will be discussed in the applications, Section 5.

Example 3.1. For $(\tau, s) \in \Delta_{2}^{T}$, assume that the kernel $K(\tau, s) \in \mathcal{L}(H)$ is given on the form $K(\tau, s)=(\tau-s)^{-\eta} A$, where $\eta \in\left(0, \frac{1}{2}\right)$ and $A \in \mathcal{L}(H)$. Furthermore, for any $\alpha \in\left(0, \frac{1}{2}\right)$ such that $\alpha>\eta$, consider an $\alpha$-Hölder continuous trajectory of an $H$-valued $Q$-Wiener process $W$. Then we can give a pathwise construction of an infinite dimensional version of what is known as the Riemann-Liouville fractional Brownian motion by setting

$$
X^{\tau}(t)=\int_{0}^{t}(\tau-s)^{-\eta} A d W(s)=\mathcal{I}\left(\Xi^{\tau}\right)(t, 0)
$$

where the integral is constructed in terms of Proposition 14. An interesting observation here is that the construction of this processes is given as a purely deterministic functional $\mathscr{I}$ applied to the Wiener process $W$, i.e. $X=\mathscr{I}(W)$. This tells us in particular that when we have constructed a Wiener processes on a probability space $(\Omega, \mathcal{F}, \mathbb{P})$, and according to Kolmogorov's continuity theorem 3 found the set $\mathcal{N}^{c} \subset \Omega$ of full measure such that 
for each $\omega \in \mathcal{N}^{c}$ the mapping $t \mapsto W(\omega, t)$ has $\alpha$-Hölder continuous trajectories for $\alpha \in\left(0, \frac{1}{2}\right)$, then the trajectory $(\tau, t) \mapsto X^{\tau}(\omega, t) \in \mathcal{V}^{(\alpha, \eta)}\left(\Delta_{2}^{T}, H\right)$. Recall in particular from Proposition 8 that the restriction mapping $t \mapsto X(\omega, t):=X^{t}(\omega, t)$ is $\rho$-Hölder continuous with $\rho=\alpha-\eta$. This illustrates the point that simply from a probabilistic construction of the Wiener process, and the identification of the set of $\mathcal{N}^{c} \subset \Omega$ on which the Wiener process is continuous, one can construct a vast class of processes $X: \mathcal{N}^{c} \times[0, T] \rightarrow H$ given by $X(\omega, t)=\mathscr{I}(W(\omega, \cdot))(t)$. In the next section we will show that under mild conditions on the deterministic operators $K$, the random variable

$$
\omega \mapsto X(\omega, t)=\mathscr{I}(W(\omega, \cdot))(t)
$$

is Gaussian on the probability space $(\Omega, \mathcal{F}, \mathbb{P})$, with an explicit covariance operator given as a two-dimensional, possibly singular, integral with respect to the covariance operator of W.

\section{Gaussian Volterra processes}

With the Sewing Lemma 10 at hand, we are now ready to investigate Volterra paths driven by Gaussian processes. The processes we consider will be constructed in a pathwise manner, as limits of Riemann-type sums through the application of Lemma 10. When the deterministic Volterra kernel $K$ is a linear operator on $H$ with sufficient regularity of the singularity, we show that these processes are again Gaussian. More specifically, we consider Volterra processes on the form

$$
X^{\tau}(t)=\int_{0}^{t} K(\tau, s) d W(s)
$$

where $\tau \geq t$ and $W$ is a Gaussian process with zero mean and a sufficiently regular covariance operator

$$
Q_{W}\left(u, u^{\prime}\right):=\mathbb{E}\left[W(u) \otimes W\left(u^{\prime}\right)\right] .
$$

Recall from Section 2 that the covariance operator is a bounded linear operator on $H$. When the Volterra kernel $K \in \mathcal{K}_{\eta}$ for some $\eta \in[0,1)$, and the covariance function $Q_{W}$ is sufficiently regular, we show that $X$ given in (4.1) is again a Gaussian process. We derive the characteristic functional of $X$, and from this give an explicit computation of the covariance structure of $X$, denoted by $Q_{X}$. In fact, we show that the covariance operator $Q_{X}$ can be written as a deterministic functional of the kernel $K$ and the covariance of $W$. That is, the covariance operator $Q_{X}$ can be written as

$$
Q_{X}=\mathscr{I}\left(K, Q_{W}\right)
$$

where $\mathscr{I}$ is an integral operator, given as a double Young-Volterra integral. Furthermore, we prove that the operator $\mathscr{I}$ is Lipschitz continuous in both of its arguments. Stability of the covariance operator tells us in particular that if we do small (sufficiently regular) perturbations of the covariance associated to a Gaussian process $W$, then the covariance associated to the Gaussian process $X$ does not change by more than the size of these perturbations. In view of statistical estimation, this demonstrates robustness of the model with respect to data.

Let us begin to motivate the construction of the integral functional $\mathscr{I}$ in (4.2). The covariance operator $Q_{X}$ associated to $X$ will be defined by the double integral from $(0,0)$ to a point $\left(t, t^{\prime}\right) \in[0, T]^{2}$ as follows

$$
Q_{X}^{\tau, \tau^{\prime}}\left(t, t^{\prime}\right)=\int_{0}^{t} \int_{0}^{t^{\prime}} K(\tau, r) d^{2} Q_{W}\left(r, r^{\prime}\right) K\left(\tau^{\prime}, r^{\prime}\right)^{*}
$$


where $K^{*}$ denotes the dual operator of $K$, and the differential $d^{2} Q_{W}$ will be given meaning below. If $Q_{W}$ is smooth, then we can think of this as given by the mixed partial derivative $d^{2} Q_{W}\left(r, r^{\prime}\right)=\frac{\partial^{2} Q_{W}}{\partial t \partial s}\left(r, r^{\prime}\right) d r d r^{\prime}$. From the proposed representation of $Q_{W}$, $Q_{X}^{\tau, \tau^{\prime}}\left(t, t^{\prime}\right) f \in H$ since $K$ and $Q_{W}$ are both bounded linear operators on $H$. At this stage, we would like to comment that the order of the integrands in (4.3) is natural when working with operator-valued integrals corresponding to covariance functions. Since the covariance operators $Q_{W}$ and $K$ are linear operators on $H$, their non-commutative nature requires special care. Indeed, first recall that for $(\tau, v, u),\left(\tau^{\prime}, v^{\prime}, u^{\prime}\right) \in \Delta_{3}^{T}$ and $f, g \in H$ we have

$$
\mathbb{E}\left[\langle K(\tau, u) W(u), f\rangle_{H}\left\langle K\left(\tau^{\prime}, v\right) W(v), g\right\rangle_{H}\right]=\mathbb{E}\left[\left\langle W(u), K(\tau, u)^{*} f\right\rangle_{H}\left\langle W(v), K\left(\tau^{\prime}, v\right)^{*} g\right\rangle_{H}\right] .
$$

Since $X$ given in (4.1) is constructed as a limit of a Riemann sum as in Proposition 14, let us motivate the construction of (4.3) by considering an approximation of $X$ given by a partition $\mathcal{P}$ of $[0, t]$ as

$$
X_{\mathcal{P}}^{\tau}(t):=\sum_{[u, v] \in \mathcal{P}} K(\tau, u)(W(v)-W(u)) .
$$

Then, the covariance operator between $X_{\mathcal{P}}^{\tau}(t)$ and $X_{\mathcal{P}^{\prime}}^{\tau^{\prime}}\left(t^{\prime}\right)$ (where $\mathcal{P}^{\prime}$ is a partition of $\left.\left[0, t^{\prime}\right]\right)$ is computed in the following way

$$
\begin{aligned}
\mathbb{E}\left[\left\langle\sum_{[u, v] \in \mathcal{P}} K(\tau, u)(W(v)-W(u)), f\right\rangle_{H}\left\langle\sum_{\left[u^{\prime}, v^{\prime}\right] \in \mathcal{P}^{\prime}} K\left(\tau^{\prime}, u^{\prime}\right)\left(W\left(v^{\prime}\right)-W\left(u^{\prime}\right)\right), g\right\rangle_{H}\right] \\
=\sum_{\substack{[u, v] \in \mathcal{P} \\
\left[u^{\prime}, v^{\prime}\right] \in \mathcal{P}^{\prime}}} \mathbb{E}\left[\left\langle(W(v)-W(u)), K(\tau, u)^{*} f\right\rangle_{H}\left\langle\left(W\left(v^{\prime}\right)-W\left(u^{\prime}\right)\right), K\left(\tau^{\prime}, u^{\prime}\right)^{*} g\right\rangle_{H}\right] \\
=\sum_{\substack{[u, v] \in \mathcal{P} \\
\left[u^{\prime}, v^{\prime}\right] \in \mathcal{P}^{\prime}}}\left\langle\square_{\left(u, u^{\prime}\right),\left(v, v^{\prime}\right)} Q_{W} K(\tau, u)^{*} f, K\left(\tau^{\prime}, u^{\prime}\right)^{*} g\right\rangle_{H} \\
=\left\langle\sum_{\substack{[u, v] \in \mathcal{P} \\
\left[u^{\prime}, v^{\prime}\right] \in \mathcal{P}^{\prime}}} K\left(\tau^{\prime}, u^{\prime}\right) \square_{\left(u, u^{\prime}\right),\left(v, v^{\prime}\right)} Q_{W} K(\tau, u)^{*} f, g\right\rangle_{H} .
\end{aligned}
$$

Here, we used the duality of linear operators and

$$
\square_{\left(u, u^{\prime}\right),\left(v, v^{\prime}\right)} Q_{W}=\mathbb{E}\left[(W(v)-W(u)) \otimes\left(W\left(v^{\prime}\right)-W\left(u^{\prime}\right)\right)\right]
$$

by recalling the definition of the increment operator $\square$ in (1.9). If $Q_{W}$ is mixeddifferentiable in its two variables, we have

$$
\square_{\left(u, u^{\prime}\right),\left(v, v^{\prime}\right)} Q_{W} \simeq \frac{\partial^{2} Q_{W}}{\partial t \partial s}(u, v)(v-u)\left(v^{\prime}-u^{\prime}\right) .
$$

whenever $u$ is close to $u^{\prime}$ and $v$ is close to $v^{\prime}$. However, we would like to allow for possibly singular covariance functions where the mixed partial derivative $\frac{\partial^{2} Q_{W}}{\partial t \partial s}$ does not exist (possibly everywhere). Thus, taking the limit when $|\mathcal{P}| \vee\left|\mathcal{P}^{\prime}\right| \rightarrow 0$ in $X_{\mathcal{P}}^{\tau}$ and $X_{\mathcal{P}}^{\tau^{\prime}}$, one would need to show that the corresponding covariance integral appearing as the limit

$$
\lim _{|\mathcal{P}| \vee\left|\mathcal{P}^{\prime}\right| \rightarrow 0} \sum_{\substack{[u, v] \in \mathcal{P} \\\left[u^{\prime}, v^{\prime}\right] \in \mathcal{P}}} K\left(\tau^{\prime}, u^{\prime}\right) \square_{\left(u, u^{\prime}\right),\left(v, v^{\prime}\right)} Q_{W} K(\tau, u)^{*}
$$

converges in $\mathcal{L}(H)$. 


\subsection{Construction of irregular covariance functions}

Our first goal will be to show the existence of the integral appearing on the righthand side of (4.3). To this end, we will give an extension of the Volterra Sewing Lemma presented in Lemma 10, to allow for two-dimensional operator-valued integrals. As the integrals we are concerned with have the very specific form given in (4.3), we will tailor the construction of the two-dimensional integral to this specific case. Our second goal is to show that the process defined in (4.1) is Gaussian if $W$ is Gaussian and $K$ is deterministic, whenever the integral on the right-hand side of (4.3) exists. Before moving on to the construction of the double integral in (4.3), we give a definition of a class of suitable two-parameter functions $Q$ which shall be used in the sequel for the construction of covariance operators.

Definition 15. Let $\alpha \in(0,1)$ and let $Q:[0, T]^{2} \rightarrow \mathcal{L}(H)$. We say that $Q$ is an $\alpha$-regular covariance operator if it satisfies

$$
\|Q\|_{\mathcal{Q}_{\alpha}}:=\|Q\|_{\alpha,(1,0)}+\|Q\|_{\alpha,(0,1)}+\|Q\|_{\alpha,(1,1)}<\infty
$$

where we define

$$
\begin{gathered}
\|Q\|_{\alpha,(1,0)}:=\sup _{\substack{(t, s) \in \Delta_{2}^{T} \\
t^{\prime} \in[0, T]}} \frac{\left\|Q\left(t, t^{\prime}\right)-Q\left(s, t^{\prime}\right)\right\|_{o p}}{|t-s|^{\alpha}} \\
\|Q\|_{\alpha,(0,1)}:=\sup _{\substack{t \in[0, T] \\
\left(t^{\prime}, s^{\prime}\right) \in \Delta_{2}^{T}}} \frac{\left\|Q\left(t, t^{\prime}\right)-Q\left(t, s^{\prime}\right)\right\|_{o p}}{\left|t^{\prime}-s^{\prime}\right|^{\alpha}} \\
\|Q\|_{\alpha,(1,1)}:=\sup _{\substack{(t, s) \in \Delta_{2}^{T} \\
\left(t^{\prime}, s^{\prime}\right) \in \Delta_{2}^{T}}} \frac{\left\|\square\left(s, s^{\prime}\right),\left(t, t^{\prime}\right) Q\right\|_{o p}}{\left[|t-s|\left|t^{\prime}-s^{\prime}\right|\right]^{\alpha}},
\end{gathered}
$$

where we recall the rectangular increment is given by

$$
\square_{\left(u, u^{\prime}\right),\left(v, v^{\prime}\right)} Q=Q\left(v, v^{\prime}\right)-Q\left(u, v^{\prime}\right)-Q\left(v, u^{\prime}\right)+Q\left(u, u^{\prime}\right) .
$$

We denote the class of all $\alpha$-regular covariance operators by $\mathcal{Q}_{\alpha}$.

The reader should notice that the space $\mathcal{Q}_{\alpha}$ of $\alpha$-regular covariance operators is larger than the space of true covariance operators $Q:[0, T]^{2} \rightarrow \mathcal{L}(H)$ (with the same path-regularity, of course). Indeed, we have $Q(t, t)$ being a symmetric and positivesemidefinite trace class operator if $Q(s, t)=\mathbb{E}[X(s) \otimes X(t)]$ is the covariance operator for a mean-zero and square-integrable $H$-valued stochastic process $X(t)$, a restriction not imposed on the elements in $\mathcal{Q}_{\alpha}$. Thus, our results in the next subsection cover a larger family of mappings $Q:[0, T]^{2} \rightarrow \mathcal{L}(H)$ than merely those which arise as covariance operators. We prefer to keep the adjective "covariance" associated to this larger class simply because we typically have such operators in mind.

Remark 16. If $Q:[0, T]^{2} \rightarrow \mathcal{L}(H)$ is 0 when one of the variables is 0 , i.e. $Q(0, t)=$ $Q(t, 0)=0$, then $Q \in \mathcal{Q}_{\alpha}$ if $\|Q\|_{\alpha,(1,1)}<\infty$. Indeed, by subtraction of $0=Q(t, 0)-Q(s, 0)$ in (4.10), we observe that

$$
\|Q\|_{\alpha,(1,0)}=\sup _{\substack{(t, s) \in \Delta_{2}^{T} \\\left(t^{\prime}, s^{\prime}\right) \in \Delta_{2}^{T}}} \frac{\left\|Q\left(t, t^{\prime}\right)-Q\left(s, t^{\prime}\right)-Q(t, 0)+Q(s, 0)\right\|_{o p}}{|t-s|^{\alpha}} \leq\|Q\|_{\alpha,(1,1)} T^{\alpha} .
$$

Similarly we can bound $\|Q\|_{\alpha,(0,1)}$. 
The space $\mathcal{Q}_{\alpha}$ is somewhat non-standard (at least from the point of view of covariance functions), and thus we provide below an example of a co-variance operator contained in this space. We consider here the covariance operator of a fractional Brownian motion, and show that it is contained in such a space. For conciseness we only consider the case of fractional Brownian motion with Hurst parameter $0<h \leq \frac{1}{2}$, as this is the case which will be discussed in later applications.

Example 4.1. Let $Q(t, s)=R^{h}(t, s) Q$ be the covariance operator of a fractional Brownian motion on a Hilbert space $H$ with Hurst parameter $h \in\left(0, \frac{1}{2}\right]$, where $R^{h}:[0, T] \rightarrow \mathbb{R}$ is given as in (2.2). Then, we have that

$$
\square_{\left(u, u^{\prime}\right),\left(v, v^{\prime}\right)} R^{h}=\frac{1}{2}\left(-\left|v-v^{\prime}\right|^{2 h}+\left|v^{\prime}-u\right|^{2 h}+\left|v-u^{\prime}\right|^{2 h}-\left|u-u^{\prime}\right|^{2 h}\right) .
$$

Using that for $\alpha \in(0,1]$, there exists a $c>0$ such that for two numbers $a, b \in \mathbb{R}$, $\left.|| a\right|^{\alpha}-|b|^{\alpha}|\leq c| a-\left.b\right|^{\alpha}$, it follows that

$$
\left|\square_{\left(u, u^{\prime}\right),\left(v, v^{\prime}\right)} R^{h}\right| \leq c|v-u|^{2 h} \wedge\left|v^{\prime}-u^{\prime}\right|^{2 h} .
$$

By using the interpolation inequality $a \wedge b \leq a^{\theta} b^{1-\theta}$ for any $\theta \in[0,1]$ and $a, b \in \mathbb{R}_{+}$, we find that

$$
\left|\square_{\left(u, u^{\prime}\right),\left(v, v^{\prime}\right)} R^{h}\right| \leq c|v-u|^{h}\left|v^{\prime}-u^{\prime}\right|^{h} .
$$

It follows that the covariance operator $R^{h}(t, s) Q$ associated to a fractional Brownian motion with Hurst parameter $h \in\left(0, \frac{1}{2}\right]$ is contained in the space $\mathcal{Q}_{h}$.

The following theorem can be viewed as an extension (or combination) of the Volterra Sewing Lemma 10 proven in [18] and the multi-parameter Sewing lemma found in [17].

Theorem 17. Let $\alpha \in(0,1), \eta \in[0,1)$ such that $\alpha-\eta>0$. Consider a covariance operator $Q:[0, T]^{2} \rightarrow \mathcal{L}(H)$ in $\mathcal{Q}_{\alpha}$, and suppose $K \in \mathcal{K}_{\eta}$ is a Volterra kernel. For partitions $\mathcal{P}$ of $[0, t]$ and $\mathcal{P}^{\prime}$ of $\left[0, t^{\prime}\right]$, define the approximating Volterra covariance function by

$$
M_{\mathcal{P} \times \mathcal{P}^{\prime}}^{\tau, \tau^{\prime}}\left(t, t^{\prime}\right):=\sum_{\substack{[u, v] \in \mathcal{P} \\\left[u^{\prime}, v^{\prime}\right] \in \mathcal{P}^{\prime}}} K(\tau, u) \square_{\left(u, u^{\prime}\right),\left(v, v^{\prime}\right)} Q K\left(\tau^{\prime}, u^{\prime}\right)^{*} .
$$

Then there exists a unique operator in $\mathcal{L}(H)$ given as the limit (in operator-norm)

$$
\mathcal{I}(K, Q)^{\tau, \tau^{\prime}}\left(t, t^{\prime}\right):=\int_{0}^{t} \int_{0}^{t^{\prime}} K(\tau, r) d^{2} Q\left(r, r^{\prime}\right) K\left(\tau^{\prime}, r^{\prime}\right)^{*}:=\lim _{\substack{|\mathcal{P}| \rightarrow 0 \\\left|\mathcal{P}^{\prime}\right| \rightarrow 0}} M_{\mathcal{P} \times \mathcal{P}^{\prime}}^{\tau, \tau^{\prime}}\left(t, t^{\prime}\right)
$$

satisfying the additivity relation

$$
\square_{\left(u, u^{\prime}\right),\left(v, v^{\prime}\right)} \mathcal{I}(K, Q)^{\tau, \tau^{\prime}}=\int_{u}^{v} \int_{u^{\prime}}^{v^{\prime}} K(\tau, r) d^{2} Q\left(r, r^{\prime}\right) K\left(\tau^{\prime}, r^{\prime}\right)^{*}
$$

Furthermore, there exists a pair $(\beta, \kappa) \in(1, \infty) \times[0,1)$ with $\beta-\kappa \geq \rho$ and a constant $C>0$ such that the following statements holds

(i). For $(\tau, t, s),\left(\tau^{\prime}, t^{\prime}, s^{\prime}\right) \in \Delta_{3}^{T}$ the following inequality holds

$$
\begin{aligned}
& \left\|\int_{s}^{t} \int_{s^{\prime}}^{t^{\prime}}(K(\tau, r)-K(\tau, s)) d^{2} Q\left(r, r^{\prime}\right)\left(K\left(\tau^{\prime}, r^{\prime}\right)^{*}-K\left(\tau^{\prime}, s^{\prime}\right)^{*}\right)\right\|_{o p} \\
& \quad \leq C\|K\|_{\mathcal{K}_{\eta}}^{2}\|Q\|_{\mathcal{Q}_{\alpha}}\left(\left[\left|\tau-t \| \tau-t^{\prime}\right|\right]^{-\kappa}\left[\left|t^{\prime}-s^{\prime}\right||t-s|\right]^{\beta}\right) \wedge\left[\left|\tau^{\prime}-s^{\prime} \| \tau-s\right|\right]^{\beta-\kappa}
\end{aligned}
$$


(ii). For $(\tau, t, s) \in \Delta_{3}^{T},\left(\tau_{1}^{\prime}, \tau_{2}^{\prime}, t^{\prime}, s^{\prime}\right) \in \Delta_{4}^{T}$ and any $\zeta \in[0, \rho)$ we have

$$
\begin{aligned}
\| \int_{s}^{t} & \int_{s^{\prime}}^{t^{\prime}}(K(\tau, r)-K(\tau, s)) d^{2} Q\left(r, r^{\prime}\right)\left(\square_{\left(\tau_{2}^{\prime}, s^{\prime}\right),\left(\tau_{1}^{\prime}, r^{\prime}\right)} K^{*}\right) \|_{o p} \\
\leq & C\|K\|_{\mathcal{K}_{\eta}}^{2}\|Q\|_{\mathcal{Q}_{\alpha}}\left|\tau_{1}^{\prime}-\tau_{2}^{\prime}\right|^{\theta} \\
\quad & \times\left|\tau_{2}^{\prime}-t^{\prime}\right|^{-\theta+\zeta}\left(\left[|\tau-t|\left|\tau-t^{\prime}\right|^{-\zeta}\right]^{-\kappa}\left[\left|t^{\prime}-s^{\prime}\right||t-s|\right]^{\beta}\right) \\
& \wedge\left[\left|\tau-s \| \tau^{\prime}-s^{\prime}\right|^{-\zeta}\right]^{\beta-\kappa} .
\end{aligned}
$$

(iii). For $\left(\tau_{1}, \tau_{2}, t, s\right) \in \Delta_{4}^{T},\left(\tau^{\prime}, t^{\prime}, s^{\prime}\right) \in \Delta_{3}^{T}$ and any $\zeta \in[0, \rho)$ we have

$$
\begin{aligned}
\| \int_{s}^{t} & \int_{s^{\prime}}^{t^{\prime}}\left(\square_{\left(\tau_{2}, s\right),\left(\tau_{1}, r\right)} K\right) d^{2} Q\left(r, r^{\prime}\right)\left(K\left(\tau^{\prime}, r^{\prime}\right)^{*}-K\left(\tau^{\prime}, s^{\prime}\right)^{*}\right) \|_{o p} \\
\leq & C\|K\|_{\mathcal{K}_{\eta}}^{2}\|Q\|_{\mathcal{Q}_{\alpha}}\left|\tau_{1}-\tau_{2}\right|^{\theta} \\
& \times\left|\tau_{2}-t\right|^{-\theta+\zeta}\left(\left[|\tau-t|\left|\tau-t^{\prime}\right|^{-\zeta}\right]^{-\kappa}\left[\left|t^{\prime}-s^{\prime}\right||t-s|\right]^{\beta}\right) \\
& \wedge\left[\left|\tau-s \| \tau^{\prime}-s^{\prime}\right|^{-\zeta}\right]^{\beta-\kappa} .
\end{aligned}
$$

(iv). For $\left(\tau_{1}, \tau_{2}, t, s\right),\left(\tau_{1}^{\prime}, \tau_{2}^{\prime}, t^{\prime}, s^{\prime}\right) \in \Delta_{4}^{T}$ and any $\zeta \in[0, \rho)$ we have

$$
\begin{aligned}
& \| \int_{s}^{t} \int_{s^{\prime}}^{t^{\prime}}\left(\square_{\left(\tau_{2}, s\right),\left(\tau_{1}, r\right)} K\right) d^{2} Q\left(r, r^{\prime}\right)\left(\square_{\left(\tau_{2}^{\prime}, s^{\prime}\right),\left(\tau_{1}^{\prime}, r^{\prime}\right)} K^{*}\right) \|_{o p} \\
& \leq C\|K\|_{\mathcal{K}_{\eta}}^{2}\|Q\|_{\mathcal{Q}_{\alpha}}\left[\left|\tau_{1}-\tau_{2}\right|\left|\tau_{1}^{\prime}-\tau_{2}^{\prime}\right|\right]^{\theta} \\
& \quad \times\left[\left|\tau_{2}-t\right|\left|\tau_{2}^{\prime}-t^{\prime}\right|\right]^{-\theta+\zeta}\left(\left[|\tau-t|\left|\tau-t^{\prime}\right|^{-\zeta}\right]^{-\kappa}\left[\left|t^{\prime}-s^{\prime}\right||t-s|\right]^{\beta}\right) \\
& \quad \wedge\left[\left|\tau-s \| \tau^{\prime}-s^{\prime}\right|^{-\zeta}\right]^{\beta-\kappa} .
\end{aligned}
$$

Proof. The first objective of this proof is to show the existence and uniqueness of the two-dimensional integral defined in (4.17). To this end, we will also encounter onedimensional integrals formed from the two-dimensional integrand $K(\tau, u) \square_{\left(u, u^{\prime}\right),\left(v, v^{\prime}\right)} Q K\left(\tau^{\prime}, u^{\prime}\right)^{*}$ used in the definition (4.16) which will be called boundary integrals. Since these integrals are simply constructed from the one-dimensional Volterra Sewing Lemma 10 in the Banach space $\mathcal{L}(H)$, we will only briefly comment on their construction here, and focus on the two-dimensional integral. The one-dimensional integrals are given on the form

$$
\begin{aligned}
\mathcal{I}_{1}^{\tau, \tau^{\prime}}\left(s, s^{\prime}, t, t^{\prime}\right): & =\int_{s}^{t} K(\tau, r)\left[d Q\left(r, t^{\prime}\right)-d Q\left(r, s^{\prime}\right)\right] K\left(\tau^{\prime}, s\right)^{*} \\
& =\int_{s}^{t} \int_{s^{\prime}}^{t^{\prime}} K(\tau, r) d^{2} Q\left(r, r^{\prime}\right) K\left(\tau^{\prime}, s\right)^{*}
\end{aligned}
$$

where we note that there is no dependence on the integration variable $r^{\prime}$ in the integrand of the second integral, and thus the integral in this variable exists naturally as an integral over a constant. The differential $d Q\left(r, t^{\prime}\right)$ with fixed second argument $t^{\prime}$ is then meant as the regular one-variable differential. We can define the second boundary integral $\mathcal{I}_{2}$ in the same way, by integrating over the interval $\left[s^{\prime}, t^{\prime}\right]$.

In the sequel, we will frequently analyse the mapping

$$
\left(s, s^{\prime}, t, t^{\prime}, \tau, \tau^{\prime}\right) \mapsto K(\tau, s) \square_{\left(s, s^{\prime}\right),\left(t, t^{\prime}\right)} Q K\left(\tau^{\prime}, s^{\prime}\right) .
$$

From time to time, we will simply write $K \square Q K^{*}$ as a generic notation. 
At this point we let $\delta^{1}$ and $\delta^{2}$ denote the $\delta$ given in (3.4) restricted to the first and third, and second and fourth variable, respectively, of a four-variable function $f\left(s, s^{\prime}, t, t^{\prime}\right)$. That is, the action on $\delta^{1}$ on $f$ for $s \leq u \leq t$ is given by

$$
\delta_{u}^{1} f\left(s, s^{\prime}, t, t^{\prime}\right)=f\left(s, s^{\prime}, t, t^{\prime}\right)-f\left(u, s^{\prime}, t, t^{\prime}\right)-f\left(s, s^{\prime}, u, t^{\prime}\right),
$$

and the action of $\delta^{2}$ is defined similarly over the variables $s^{\prime} \leq u^{\prime} \leq t^{\prime}$. Then, using the Volterra Sewing Lemma 10 we can show that there exists a pair $(\beta, \kappa) \in(1, \infty) \times[0,1)$ such that

$$
\begin{aligned}
& \left\|\mathcal{I}_{1}^{\tau, \tau^{\prime}}\left(s, s^{\prime}, t, t^{\prime}\right)-K(\tau, s) \square_{\left(s, s^{\prime}\right),\left(t, t^{\prime}\right)} Q K\left(\tau^{\prime}, s^{\prime}\right)^{*}\right\|_{o p} \\
& \quad \leq C\|\| \delta^{1}\left(K(\cdot, \cdot) \square_{\left(\cdot, s^{\prime}\right),\left(\cdot, t^{\prime}\right)} Q K\left(\tau^{\prime}, s^{\prime}\right)^{*}\right)\|\|_{(\beta, \kappa), 1}\left[|\tau-t|^{-\kappa}|t-s|^{\beta}\right] \wedge|\tau-s|^{\beta-\kappa}
\end{aligned}
$$

Indeed, in order to apply Lemma 10 we need to show that $\delta^{1}$ acting on the increment $K \square Q K^{*}$ is sufficiently regular. By elementary algebraic manipulations, we observe in particular that

$$
\delta_{z}^{1} K(\tau, u) \square_{\left(u, s^{\prime}\right),(v, t)} Q K\left(\tau^{\prime}, s^{\prime}\right)^{*}=(K(\tau, u)-K(\tau, z)) \square_{\left(z, s^{\prime}\right),(v, t)} Q K\left(\tau^{\prime}, s^{\prime}\right)^{*} .
$$

With this relation at hand, invoking the assumption that $K \in \mathcal{K}_{\eta}$ and the regularity condition on $Q$ given by (4.10), we obtain that for any $\theta \in[0,1]$

$$
\begin{aligned}
& \left\|\delta_{z}^{1} K(\tau, u) \square_{\left(u, s^{\prime}\right),\left(v, t^{\prime}\right)} Q K\left(\tau^{\prime}, s^{\prime}\right)^{*}\right\|_{o p} \\
& \quad \leq\|K\|_{\eta, 3}\|Q\|_{\alpha,(1,1)}\|K\|_{\eta, 1}|\tau-z|^{-\eta-\theta}|v-u|^{\alpha+\theta} T^{\alpha-\eta}
\end{aligned}
$$

Here we have used that

$$
\left\|\square_{\left(u, s^{\prime}\right),\left(v, t^{\prime}\right)} Q\right\|_{o p} \leq|v-u|^{\alpha}\left|t^{\prime}-s^{\prime}\right|^{\alpha} \quad \text { and } \quad\left\|K\left(\tau^{\prime}, s^{\prime}\right)\right\|_{o p} \leq\left|\tau^{\prime}-s^{\prime}\right|^{-\eta},
$$

and thus

$$
\left\|\square_{\left(u, s^{\prime}\right),\left(v, t^{\prime}\right)} Q\right\|_{o p}\left\|K\left(\tau^{\prime}, s^{\prime}\right)\right\|_{o p} \leq|v-u|^{\alpha} T^{\alpha-\eta},
$$

where $\rho=\alpha-\eta$ In the same way, one can verify a similar bound for $\delta^{2} K \square Q K^{*}$. It follows that that the bound in (4.25) holds by setting $\beta=\alpha+\theta$ and $\kappa=\eta+\theta$ and choosing $\theta \in[0,1]$ such that $(\beta, \kappa) \in(1, \infty) \times[0,1)$ (which is always possible due to the fact that $\eta<\alpha$ ). The fact that $C$ in (4.25) can be chosen uniformly in all the time variables, follows from the assumption that $\eta<\alpha$, which in particular implies that any singularity coming from $K$ as $s^{\prime} \rightarrow \tau^{\prime}$ is killed by the regularity of $\square_{\left(\cdot, s^{\prime}\right),\left(\cdot, t^{\prime}\right)} Q$, since $s^{\prime} \leq t^{\prime} \leq \tau^{\prime}$. Similarly, we can define the integral $\mathcal{I}_{2}$ in the same way by letting the integrand be independent of the first integration variable $r$. By the same analysis as above, with application of the one-dimensional Volterra Sewing Lemma 10, one gets that also $\mathcal{I}_{2}$ is a well-defined integral, satisfying a similar bound to (4.25).

Now let us focus on the two-dimensional integral operator $\mathcal{I}$ in (4.17). First, we note that the additivity relation in (4.18) is a straightforward consequence of the additivity of the limit of the one-dimensional Riemann sum, corresponding to the property

$$
\int_{0}^{t} f(r) d r-\int_{0}^{s} f(r) d r=\int_{s}^{t} f(r) d r .
$$

See [14] or [17] for more details on this property in connection with the Sewing lemma both in the one-parameter and multi-parameter setting.

For the uniqueness of the integral defined in (4.17), assume for now that the integral exists and satisfies (i)-(iv), and consider the following argument: Assume $\mathcal{M}$ and $\overline{\mathcal{M}}$ are two candidates for $\mathcal{I}$, both constructed from the integrand $K \square Q K^{*}$. We obtain for 
both $\mathcal{M}$ and $\overline{\mathcal{M}}$ one-dimensional integrals, which are unique by the one-dimensional sewing lemma. Thus, the boundary integrals $\mathcal{I}_{1}$ and $\mathcal{I}_{2}$ of both are identical. Note that this implies in particular that the boundary integrals corresponding to the difference $\mathcal{M}-\overline{\mathcal{M}}$ is equal to 0 . Invoking this fact, it follows from (4.19) that the increment $\square_{\left(s, s^{\prime}\right),\left(t, t^{\prime}\right)}(\mathcal{M}-\overline{\mathcal{M}})$ satisfies the following bound for $s \leq t \leq \tau$ and $s^{\prime} \leq t^{\prime} \leq \tau^{\prime}$ and some $(\beta, \kappa) \in(1, \infty) \times[0,1)$

$$
\left\|\square_{\left(s, s^{\prime}\right),\left(t, t^{\prime}\right)}(\mathcal{M}-\overline{\mathcal{M}})\right\|_{o p} \leq C\left[|\tau-t|\left|\tau-t^{\prime}\right|\right]^{-\kappa}\left[\left|t^{\prime}-s^{\prime}\right||t-s|\right]^{\beta} .
$$

Furthermore, due to the additive property in (4.18) of the increment we have that

$$
\mathcal{M}^{\tau, \tau \prime}\left(s, s^{\prime}, t, t^{\prime}\right)-\overline{\mathcal{M}}^{\tau, \tau \prime}\left(s, s^{\prime}, t, t^{\prime}\right)=\sum_{\substack{[u, v] \in \mathcal{P} \\\left[u^{\prime}, v^{\prime}\right] \in \mathcal{P}^{\prime}}} \square_{\left(u, u^{\prime}\right),\left(v, v^{\prime}\right)}\left[\mathcal{M}^{\tau, \tau \prime}-\overline{\mathcal{M}}^{\tau, \tau \prime}\right]
$$

where $\mathcal{P}$ and $\mathcal{P}^{\prime}$ are now partitions of $[s, t]$ and $\left[s^{\prime}, t^{\prime}\right]$ respectively. Thanks to (4.30) it follows that we can bound the left hand side of (4.31) in the following way

$$
\begin{aligned}
\left\|\mathcal{M}^{\tau, \tau \prime}\left(0,0, t, t^{\prime}\right)-\overline{\mathcal{M}}^{\tau, \tau \prime}\left(0,0, t, t^{\prime}\right)\right\|_{o p} & \leq C \sum_{\substack{[u, v] \in \mathcal{P} \\
\left[u^{\prime}, v^{\prime}\right] \in \mathcal{P}^{\prime}}}\left[\left|\tau-v \| \tau-v^{\prime}\right|\right]^{-\kappa}\left[\left|v^{\prime}-u^{\prime} \| v-u\right|\right]^{\beta} \\
& \leq C\left[\left|\mathcal{P} \| \mathcal{P}^{\prime}\right|\right]^{\beta-1} \int_{s}^{t} \int_{s^{\prime}}^{t^{\prime}}\left[\left|\tau-r \| \tau^{\prime}-r^{\prime}\right|\right]^{-\kappa} d r^{\prime} d r
\end{aligned}
$$

where we have appealed to the restriction of the parameters $(\beta, \kappa) \in(1, \infty) \times[0,1)$ in the last inequality, as well as recalling that $|\mathcal{P}|$ denotes the size of the mesh of the partition $\mathcal{P}$. We can now choose the partition arbitrarily fine, which implies that the difference $\mathcal{M}^{\tau, \tau \prime}\left(0,0, t, t^{\prime}\right)-\overline{\mathcal{M}}^{\tau, \tau \prime}\left(0,0, t, t^{\prime}\right) \equiv 0$. We conclude that the integral constructed in (4.17) is unique.

We continue with the proof of the existence of the integral $\mathcal{I}(K, Q)$ given in (4.17). To shorten slightly the notation, we from now on write $\mathcal{I}:=\mathcal{I}(K, Q)$ for the two-dimensional integral. In our argument, we first consider a sequence of approximating integrals constructed from dyadic partitions, and show that this sequence is Cauchy. We show in particular that the integral constructed from dyadic partitions satisfy the regularity condition in (i). Second, we show that the definition may be extended to any partition, and thus the limit in Equation (4.17) is independent of the chosen partition $\mathcal{P}$.

Consider now dyadic partitions $\mathcal{P}^{n}$ for $n \in \mathbb{N}_{0}$ defined in the following way: $\mathcal{P}^{0}=$ $\{[s, t]\}$ and $\mathcal{P}^{n}$ is defined iteratively for $n \geq 1$ by

$$
\mathcal{P}^{n}:=\bigcup_{[u, v] \in \mathcal{P}^{n-1}}\{[u, z],[z, v]\},
$$

where the point $z=(v+u) / 2$. It is readily checked that each interval $[u, v] \in \mathcal{P}^{n}$ is of length $2^{-n}|t-s|$, and that $\mathcal{P}^{n}$ consists of $2^{n}$ intervals. Construct the dyadic partition $\mathcal{P}^{\prime, n^{\prime}}$ of $\left[s^{\prime}, t^{\prime}\right]$ similarly for $n^{\prime} \in \mathbb{N}_{0}$.

For $n, n^{\prime} \in \mathbb{N}$, observe that

$$
M_{\mathcal{P}^{n} \times \mathcal{P}^{\prime, n^{\prime}}}^{\tau, \gamma^{\prime}}-M_{\mathcal{P}^{n} \times \mathcal{P}^{\prime}, n^{\prime}-1}^{\tau, \tau^{\prime}}=\sum_{\substack{[u, v] \in \mathcal{P}^{n} \\\left[u^{\prime}, v^{\prime}\right] \in \mathcal{P}^{\prime, n^{\prime}-1}}} \delta_{z^{\prime}}^{2} K(\tau, u) \square_{\left(u, u^{\prime}\right),\left(v, v^{\prime}\right)} Q K\left(\tau^{\prime}, u^{\prime}\right)^{*} .
$$

From this we see that the following relation holds

$$
M_{\mathcal{P}^{n} \times \mathcal{P}^{\prime}, n^{\prime}}^{\tau, \tau^{\prime}}-M_{\mathcal{P}^{n} \times \mathcal{P}^{\prime}, n^{\prime}-1}^{\tau, \tau^{\prime}}-M_{\mathcal{P}^{n-1} \times \mathcal{P}^{\prime}, n^{\prime}}^{\tau, \tau^{\prime}}+M_{\mathcal{P}^{n-1} \times \mathcal{P}^{\prime, n^{\prime}-1}}^{\tau, \tau^{\prime}}
$$


Infinite dimensional pathwise Volterra processes

$$
=\sum_{\substack{[u, v] \in \mathcal{P}^{n-1} \\\left[u^{\prime}, v^{\prime}\right] \in \mathcal{P}^{\prime, n^{\prime}-1}}} \delta_{z}^{1} \delta_{z^{\prime}}^{2} K(\tau, u) \square_{\left(u, u^{\prime}\right),\left(v, v^{\prime}\right)} Q K\left(\tau^{\prime}, u^{\prime}\right)^{*} .
$$

Here $\delta^{1} \delta^{2}=\delta^{1} \circ \delta^{2}=\delta^{2} \circ \delta^{1}$ is the composition of the one-dimensional delta's given in (4.24). We have already seen the action of $\delta^{i}$ for $i=1,2$ applied to the increment $K \square Q K^{*}$ in (4.26), and we will therefore now compute the action of the composition $\delta^{1} \delta^{2}$. We get for $(v, z, u),\left(v^{\prime}, z^{\prime}, u^{\prime}\right) \in \Delta_{3}^{T}$ that

$$
\begin{aligned}
& \delta_{z}^{1} \delta_{z^{\prime}}^{2} K(\tau, u) \square_{\left(u, u^{\prime}\right),\left(v, v^{\prime}\right)} Q K\left(\tau^{\prime}, u^{\prime}\right)^{*} \\
& \quad=(K(\tau, u)-K(\tau, z)) \square_{\left(z, z^{\prime}\right),\left(v, v^{\prime}\right)} Q\left(K\left(\tau^{\prime}, u^{\prime}\right)-K\left(\tau^{\prime}, z^{\prime}\right)\right)^{*}
\end{aligned}
$$

Using the relation in (4.35) together with the assumption that $K, K^{*} \in \mathcal{K}_{\eta}$ and that the covariance $Q$ is $\alpha$-regular, we obtain the following bound for any $\theta \in[0,1]$

$$
\begin{aligned}
& \left\|\delta_{z}^{1} \delta_{z^{\prime}}^{2} K(\tau, u) \square_{\left(u, u^{\prime}\right),\left(v, v^{\prime}\right)} Q K\left(\tau^{\prime}, u^{\prime}\right)^{*}\right\|_{o p} \\
& \quad \leq\|K\|_{\eta, 3}^{2}\|Q\|_{\alpha,(1,1)}\left[\left|\tau-z \| \tau^{\prime}-z^{\prime}\right|\right]^{-\eta-\theta}\left[\left|v-u \| v^{\prime}-u^{\prime}\right|\right]^{\alpha+\theta}
\end{aligned}
$$

where we have used that $|z-u|^{\theta} \leq|v-u|^{\theta}$, and similarly for the difference $\left|z^{\prime}-u^{\prime}\right|^{\theta}$. With this inequality at hand, we will now go back to the difference in (4.34). By telescoping sums, we observe that for $n^{\prime}>m^{\prime}$ we have

$$
M_{\mathcal{P}^{n} \times \mathcal{P}^{\prime}, n^{\prime}}^{\tau, \tau^{\prime}}-M_{\mathcal{P}^{n} \times \mathcal{P}^{\prime}, m^{\prime}}^{\tau, \tau^{\prime}}=\sum_{i=m^{\prime}+1}^{n^{\prime}} M_{\mathcal{P}^{n} \times \mathcal{P}^{\prime, i}}^{\tau, \tau^{\prime}}-M_{\mathcal{P}^{n} \times \mathcal{P}^{\prime, i-1}}^{\tau, \tau^{\prime}}
$$

with the same type of relation for the difference $M_{\mathcal{P}^{n} \times \mathcal{P}^{\prime}, n^{\prime}}^{\tau, \tau^{\prime}}-M_{\mathcal{P}^{m} \times \mathcal{P}^{\prime}, n^{\prime}}^{\tau, \tau^{\prime}}$ when $n>m$. Combining the two and inserting the relation in (4.35) yields that

$$
\begin{aligned}
M_{\mathcal{P}^{n} \times \mathcal{P}^{\prime}, n^{\prime}}^{\tau, \tau^{\prime}}-M_{\mathcal{P}^{n} \times \mathcal{P}^{\prime, m^{\prime}}}^{\tau, \tau^{\prime}}-M_{\mathcal{P}^{m} \times \mathcal{P}^{\prime, n^{\prime}}}^{\tau, \tau^{\prime}}+M_{\mathcal{P}^{m} \times \mathcal{P}^{\prime}, m^{\prime}}^{\tau, \tau^{\prime}} & \sum_{\substack{i \in\{m+1, \ldots, n\} \\
j \in\left\{m^{\prime}+1, \ldots, n^{\prime}\right\}}} \sum_{\substack{[u, v] \in \mathcal{P}^{i} \\
\left[u^{\prime}, v^{\prime}\right] \in \mathcal{P}^{\prime, j}}} \delta_{z}^{1} \delta_{z^{\prime}}^{2} K(\tau, z) \square_{\left(u, u^{\prime}\right),\left(v, v^{\prime}\right)} Q K\left(\tau^{\prime}, z^{\prime}\right)^{*}
\end{aligned}
$$

Invoking the inequality obtained in (4.36), we can bound the left-hand side of (4.38) in the following way

$$
\begin{aligned}
& \| M_{\mathcal{P}^{n} \times \mathcal{P}^{\prime, n^{\prime}}}^{\tau, \tau^{\prime}}-M_{\mathcal{P}^{n} \times \mathcal{P}^{\prime, m^{\prime}}}^{\tau, \tau^{\prime}}-M_{\mathcal{P}^{m} \times \mathcal{P}^{\prime}, n^{\prime}}^{\tau, \tau^{\prime}}+M_{\mathcal{P}^{m} \times \mathcal{P}^{\prime}, m^{\prime}}^{\tau, \tau_{o p}^{\prime}} \\
& \leq\|K\|_{\eta, 3}^{2}\|Q\|_{\alpha,(1,1)} \sum_{\substack{i \in\{m+1, \ldots, n\} \\
j \in\left\{m^{\prime}+1, \ldots, n^{\prime}\right\}}} \sum_{\substack{[u, v] \in \mathcal{P}^{i} \\
\left[u^{\prime}, v^{\prime}\right] \in \mathcal{P}^{\prime, j}}}\left[\left|\tau-z \| \tau^{\prime}-z^{\prime}\right|\right]^{-\eta-\theta}\left[\left|v-u \| v^{\prime}-u^{\prime}\right|\right]^{\alpha+\theta} \\
& \quad=: V_{\mathcal{P}, \mathcal{P}^{\prime}} .
\end{aligned}
$$

Then we choose $\kappa=\theta+\eta$ and $\beta=\theta+\alpha$ such that $(\beta, \kappa) \in(1, \infty) \times[0,1)$ (again we note that this is always possible since $\alpha-\eta>0)$. Using that for any $[v, u] \in \mathcal{P}^{n}$ it holds $|v-u|=2^{-n}|t-s|$, we obtain that

$$
\begin{aligned}
& V_{\mathcal{P}, \mathcal{P}^{\prime}} \leq C R\left(m, n, m^{\prime}, n^{\prime}\right)\left[\left|t-s \| t^{\prime}-s^{\prime}\right|\right]^{\beta-1} \int_{s}^{t} \int_{s^{\prime}}^{t^{\prime}}\left[\left|\tau-r \| \tau^{\prime}-r^{\prime}\right|\right]^{-\kappa} d r^{\prime} d r \\
& \leq C R\left(m, n, m^{\prime}, n^{\prime}\right)\left(\left[\left|\tau-t \| \tau^{\prime}-t^{\prime}\right|\right]^{-\kappa}\left[\left|t-s \| t^{\prime}-s^{\prime}\right|\right]^{\beta}\right) \wedge\left[\left|\tau-s \| \tau^{\prime}-s^{\prime}\right|\right]^{\beta-\kappa}
\end{aligned}
$$


where we have used $R\left(m, n, m^{\prime}, n^{\prime}\right):=\sum_{\substack{i \in\{m+1, \ldots, n\} \\ j \in\left\{m^{\prime}+1, \ldots, n^{\prime}\right\}}} 2^{-(i+j)(\beta-1)}$. Inserting (4.40) into the right hand side of (4.39), it follows that $\left\{M_{\mathcal{P}^{n} \times \mathcal{P}^{\prime}, n^{\prime}}\right\}_{\left(n, n^{\prime}\right) \in \mathbb{N}^{2}}$ is a Cauchy sequence (with multi-index $\left(n, n^{\prime}\right) \in \mathbb{N}$ ). We define the limit of this sequence as $n, n^{\prime} \rightarrow \infty$ to be

$$
\mathcal{I}^{\tau, \tau^{\prime}}\left(s, s^{\prime}, t, t^{\prime}\right):=\lim _{n, n^{\prime} \rightarrow \infty} M_{\mathcal{P}^{n} \times \mathcal{P}^{\prime, n^{\prime}}}^{\tau, \tau^{\prime}}
$$

It now follows directly from the additivity property (4.18) proven in the beginning of the proof that the following identity holds

$$
\mathcal{I}^{\tau, \tau^{\prime}}\left(s, s^{\prime}, t, t^{\prime}\right)=\square_{\left(s, s^{\prime}\right),\left(t, t^{\prime}\right)} \mathcal{I}^{\tau, \tau^{\prime}}(0,0, \cdot, \cdot) .
$$

Furthermore, due to the relations (4.27) and (4.37), and by deriving a one-dimensional estimate similar to (4.39), it follows that the boundary terms $\left\{M_{\mathcal{P}^{n} \times\left[s^{\prime}, t^{\prime}\right]}^{\tau, \tau^{\prime}}\right\}_{n \in \mathbb{N}}$ and $\left\{M_{[s, t] \times \mathcal{P}^{\prime}, n^{\prime}}^{\tau, \tau^{\prime}}\right\}_{n^{\prime} \in \mathbb{N}}$ are both Cauchy sequences. Moreover, we observe that the boundary integrals are given as

$$
\begin{aligned}
& \mathcal{I}_{1}^{\tau, \tau^{\prime}}\left(s, s^{\prime}, t, t^{\prime}\right)=\lim _{n \rightarrow \infty} M_{\mathcal{P}^{n} \times\left[s^{\prime}, t^{\prime}\right]}^{\tau, \tau^{\prime}}, \\
& \mathcal{I}_{2}^{\tau, \tau^{\prime}}\left(s, s^{\prime}, t, t^{\prime}\right)=\lim _{n^{\prime} \rightarrow \infty} M_{[s, t] \times \mathcal{P}^{\prime}, n^{\prime}}^{\tau, \tau^{\prime}} .
\end{aligned}
$$

Note that these two objects are only additive in one pair of its variables, i.e. we have

$$
\mathcal{I}_{1}^{\tau, \tau^{\prime}}\left(0, s^{\prime}, t, t^{\prime}\right)-\mathcal{I}_{1}^{\tau, \tau^{\prime}}\left(0, s^{\prime}, s, t^{\prime}\right)=\mathcal{I}_{1}^{\tau, \tau^{\prime}}\left(s, s^{\prime}, t, t^{\prime}\right)
$$

while on the other hand

$$
\mathcal{I}_{1}^{\tau, \tau^{\prime}}\left(s, 0, t, t^{\prime}\right)-\mathcal{I}_{1}^{\tau, \tau^{\prime}}\left(s, 0, t, s^{\prime}\right) \neq \mathcal{I}_{1}^{\tau, \tau^{\prime}}\left(s, s^{\prime}, t, t^{\prime}\right) .
$$

The opposite relation holds for $\mathcal{I}_{2}$. This is due to the nature of the integrand $K \square Q K^{*}$ and since

$$
\mathcal{I}_{1}^{\tau, \tau^{\prime}}\left(s, s^{\prime}, t, t^{\prime}\right)=\lim _{n \rightarrow \infty} M_{\mathcal{P}^{n} \times\left[s^{\prime}, t^{\prime}\right]}^{\tau, \tau^{\prime}}=\lim _{n \rightarrow \infty} \sum_{[u, v] \in \mathcal{P}^{n}} K(\tau, u) \square_{u, s^{\prime}, v, t^{\prime}} Q K\left(\tau^{\prime}, s^{\prime}\right)^{*} .
$$

Recall that the boundary integrals $\mathcal{I}_{1}$ should be thought of as the integral appearing in (4.23), and similarly for $\mathcal{I}_{2}$. Now, we observe from (4.39) with $m=m^{\prime}=0$ that if we let

$$
\begin{aligned}
& \mathcal{H}^{\tau, \tau^{\prime}}\left(s, s^{\prime}, t, t^{\prime}\right) \\
& :=\mathcal{I}^{\tau, \tau^{\prime}}\left(s, s^{\prime}, t, t^{\prime}\right)-\mathcal{I}_{1}^{\tau, \tau^{\prime}}\left(s, s^{\prime}, t, t^{\prime}\right)-\mathcal{I}_{2}^{\tau, \tau^{\prime}}\left(s, s^{\prime}, t, t^{\prime}\right)+K(\tau, s) \square_{\left(s, s^{\prime}\right),\left(t, t^{\prime}\right)} Q K\left(\tau^{\prime}, s^{\prime}\right)^{*}
\end{aligned}
$$

then we have

$$
\begin{aligned}
& \left\|\mathcal{H}^{\tau, \tau^{\prime}}\left(s, s^{\prime}, t, t^{\prime}\right)\right\|_{o p} \leq C\|K\|_{\eta, 3}^{2}\|Q\|_{\alpha,(1,1)} \\
& \quad\left[\frac{1}{1-2^{1-\beta}}\right]^{2}\left(\left[|\tau-t|\left|\tau^{\prime}-t^{\prime}\right|\right]^{-\kappa}\left[\left|t-s \| t^{\prime}-s^{\prime}\right|\right]^{\beta}\right) \wedge\left[\left|\tau-s \| \tau^{\prime}-s^{\prime}\right|\right]^{\beta-\kappa},
\end{aligned}
$$

where $(\beta, \kappa) \in(1, \infty) \times[0,1)$ is chosen according to the rules specified below (4.39). We conclude that the limiting objects $\mathcal{I}, \mathcal{I}_{1}$ and $\mathcal{I}_{2}$ made from limits of dyadic partitions exist uniquely and satisfy the regularity condition in (4.19).

The reader would note that we can construct the limiting object $\mathcal{I}$ as a limit of a Riemann sum over either $\mathcal{I}_{1}$ or $\mathcal{I}_{2}$. By this we mean that the two-dimensional integral, 
can be obtained as a limit of a Riemann sum over the one-dimensional boundary integral. In particular we have that

$$
\mathcal{I}^{\tau, \tau^{\prime}}\left(s, s^{\prime}, t, t^{\prime}\right)=\lim _{n^{\prime} \rightarrow \infty} \sum_{\left[u^{\prime}, v^{\prime}\right] \in \mathcal{P}^{\prime}, n^{\prime}} \mathcal{I}_{1}^{\tau, \tau^{\prime}}\left(s, u^{\prime}, t, v^{\prime}\right),
$$

and similarly for $\mathcal{I}_{2}$ where integration is done over a dyadic partition of $[s, t]$. Indeed, recall that $\mathcal{I}_{1}$ is additive in the first variable, by which we mean that for any partition $\mathcal{P}$ we have

$$
\mathcal{I}_{1}^{\tau, \tau^{\prime}}\left(s, s^{\prime}, t, t^{\prime}\right)=\sum_{[u, v] \in \mathcal{P}} \mathcal{I}_{1}^{\tau, \tau^{\prime}}\left(u, s^{\prime}, v, t^{\prime}\right)
$$

A similar property holds for $\mathcal{I}_{2}$. Given the structure of the integrand $K \square Q K^{*}$, and in the spirit of Lemma 10 together with linearity of the integral it is readily checked that for any $\theta \in[0,1]$

$$
\left\|\delta_{m^{\prime}}^{2} \mathcal{I}_{1}^{\tau, \tau^{\prime}}\left(s, u^{\prime}, t, v^{\prime}\right)\right\|_{o p} \leq C\|K\|_{\eta, 1}\|Q\|_{\alpha,(0,1)}\|K\|_{\eta, 2}\left[|\tau-m|^{-\eta-\theta}\left|v^{\prime}-u^{\prime}\right|^{\alpha+\theta}\right]|\tau-s|^{-\eta}|t-s|^{\alpha},
$$

where the estimate is uniform in the ordered variables $(s, t, \tau)$. Then again setting $\beta=\rho+\theta$ and $\kappa=\eta+\theta$ and choosing $\theta \in[0,1]$ such that $(\beta, \kappa) \in(1, \infty) \times[0,1)$, and next invoking (4.43) together with (4.44), it follows from the Volterra Sewing Lemma 10 that

$$
\begin{aligned}
& \left\|\mathcal{I}^{\tau, \tau^{\prime}}\left(s, s^{\prime}, t, t^{\prime}\right)-\mathcal{I}_{1}^{\tau, \tau^{\prime}}\left(s, s^{\prime}, t, t^{\prime}\right)\right\|_{o p} \\
& \left.\quad \leq C\|K\|_{\eta, 1}\|Q\|_{\alpha,(0,1)}\|K\|_{\eta, 2}\left|\tau-t^{\prime}\right|^{-\kappa}\left|t^{\prime}-s^{\prime}\right|^{\beta} \wedge\left|\tau-s^{\prime}\right|^{\beta-\kappa}\right]|\tau-s|^{-\eta}|t-s|^{\alpha},
\end{aligned}
$$

and thus relation (4.42) follows directly. Similarly, one can show that

$$
\begin{aligned}
\| \mathcal{I}^{\tau, \tau^{\prime}}\left(s, s^{\prime}, t, t^{\prime}\right) & -\mathcal{I}_{2}^{\tau, \tau^{\prime}}\left(s, s^{\prime}, t, t^{\prime}\right) \|_{o p} \\
& \left.\leq C\|K\|_{\eta, 2}\|Q\|_{\alpha,(1,0)}\|K\|_{\eta, 1}|\tau-t|^{-\kappa}|t-s|^{\beta} \wedge|\tau-s|^{\beta-\kappa}\right]\left|\tau^{\prime}-s^{\prime}\right|^{-\eta}\left|t^{\prime}-s^{\prime}\right|^{\alpha} .
\end{aligned}
$$

Our next goal is to show that the limiting object $\mathcal{I}$ is independent of the chosen partition $\mathcal{P}$. Note that the one-dimensional integral terms are in fact independent of the partition chosen, as a consequence of the one-dimensional Volterra Sewing Lemma 10. Therefore, following from the relation (4.42), it is sufficient to show that the differences

$$
\begin{aligned}
& \mathcal{I}^{\tau, \tau^{\prime}}\left(s, s^{\prime}, t, t^{\prime}\right)-\sum_{[u, v] \in \mathcal{P}^{\prime}} \mathcal{I}_{1}^{\tau, \tau^{\prime}}\left(s, u^{\prime}, t, v^{\prime}\right), \\
& \mathcal{I}^{\tau, \tau^{\prime}}\left(s, s^{\prime}, t, t^{\prime}\right)-\sum_{[u, v] \in \mathcal{P}} \mathcal{I}_{2}^{\tau, \tau^{\prime}}\left(u, s^{\prime}, v, t^{\prime}\right),
\end{aligned}
$$

converge to zero for generic partitions $\mathcal{P}^{\prime}$ and $\mathcal{P}$, where $\left|\mathcal{P}^{\prime}\right| \rightarrow 0$ and $|\mathcal{P}| \rightarrow 0$. Let us prove this for (4.46). The same result for (4.47) can be found by an analogous procedure. By additivity of $\mathcal{I}$ and $\mathcal{I}_{1}$, we can write

$$
\mathcal{I}^{\tau, \tau^{\prime}}\left(s, s^{\prime}, t, t^{\prime}\right)-\sum_{[u, v] \in \mathcal{P}^{\prime}} \mathcal{I}_{1}^{\tau, \tau^{\prime}}\left(s, u^{\prime}, t, v^{\prime}\right)=\sum_{[u, v] \in \mathcal{P}^{\prime}} \mathcal{I}^{\tau, \tau^{\prime}}\left(s, u^{\prime}, t, v^{\prime}\right)-\mathcal{I}_{1}^{\tau, \tau^{\prime}}\left(s, u^{\prime}, t, v^{\prime}\right)
$$

Invoking the bounds we found in (4.45), we can majorize the right-hand side of (4.48), which yields that

$$
\begin{aligned}
\left\|\mathcal{I}^{\tau, \tau^{\prime}}\left(s, s^{\prime}, t, t^{\prime}\right)-\sum_{[u, v] \in \mathcal{P}^{\prime}} \mathcal{I}_{1}^{\tau, \tau^{\prime}}\left(s, u^{\prime}, t, v^{\prime}\right)\right\|_{o p} & \leq C \sum_{\left[u^{\prime}, v^{\prime}\right] \in \mathcal{P}^{\prime}}\left|\tau^{\prime}-v^{\prime}\right|^{-\kappa}\left|u^{\prime}-v^{\prime}\right|^{\beta} \\
& \leq C\left|\mathcal{P}^{\prime}\right|^{\beta-1} \int_{s^{\prime}}^{t^{\prime}}\left|\tau^{\prime}-r\right|^{-\kappa} d r
\end{aligned}
$$


where the integral is convergent since $\kappa<1$, and the constant $C>0$ may depend on $T^{\rho}$. Thus, letting $\left|\mathcal{P}^{\prime}\right| \rightarrow 0$ we observe that

$$
\left\|\mathcal{I}^{\tau, \tau^{\prime}}\left(s, s^{\prime}, t, t^{\prime}\right)-\sum_{[u, v] \in \mathcal{P}^{\prime}} \mathcal{I}_{1}^{\tau, \tau^{\prime}}\left(s, u^{\prime}, t, v^{\prime}\right)\right\|_{o p} \rightarrow 0,
$$

since $\beta>1$, and we conclude that the integral $\mathcal{I}$ in (4.17) is independent of the choice of partition. We conclude that the limit in (4.17) exists uniquely, and it follows from (4.41) that the inequality in (i) holds.

It now remains to show that also (ii)-(iv) holds. From the proof above, all the integrals appearing in these expressions exist, and so the different regularity estimates differ from (i) in the sense that they have various increments in the upper parameters of the Volterra kernels. As the proof of these inequalities are essentially identical with the proof of (i) above, we will only show the inequality in (iv) here, and leave the details for (ii)-(iii) to the reader. This we do because (ii)-(iii) can be seen as mixtures of (i) and (iv), and it will therefore be simple to verify that also these inequalities hold. To illustrate this point, for $\left(\tau_{1}, \tau_{2}, t, s\right),\left(\tau_{1}^{\prime}, \tau_{2}^{\prime}, t^{\prime}, s^{\prime}\right) \in \Delta_{4}^{T}$ define $G^{\tau_{1}, \tau_{2}}(r)=K\left(\tau_{1}, r\right)-K\left(\tau_{2}, r\right)$, and observe that

$$
\begin{aligned}
& \int_{s}^{t} \int_{s^{\prime}}^{t^{\prime}}(\left.\square_{\left(\tau_{2}, s\right),\left(\tau_{1}, r\right)} K\right) d^{2} Q\left(r, r^{\prime}\right)\left(\square_{\left(\tau_{2}^{\prime}, s^{\prime}\right),\left(\tau_{1}^{\prime}, r^{\prime}\right)} K^{*}\right) \\
& \quad=\int_{s}^{t} \int_{s^{\prime}}^{t^{\prime}}\left(G^{\tau_{1}, \tau_{2}}(r)-G^{\tau_{1}, \tau_{2}}(s)\right) d^{2} Q\left(r, r^{\prime}\right)\left(G^{\tau_{1}^{\prime}, \tau_{2}^{\prime}}\left(r^{\prime}\right)^{*}-G^{\tau_{1}^{\prime}, \tau_{2}^{\prime}}\left(s^{\prime}\right)^{*}\right) .
\end{aligned}
$$

The right-hand side is an integral expression on the same form as in in (i), however with different Volterra kernel. Similarly, we observe that (ii) and (iii) can be written as mixtures of integrals over the kernels $K$ and $G$ defined above. Following the strategy outlined above to prove (i), we now consider the integrand

$G^{\tau_{1}, \tau_{2}}(s) \square_{\left(s, s^{\prime}\right),\left(t, t^{\prime}\right)} Q G^{\tau_{1}^{\prime}, \tau_{2}^{\prime}}\left(s^{\prime}\right)=\left(K\left(\tau_{1}, s\right)-K\left(\tau_{2}, s\right)\right) \square_{\left(s, s^{\prime}\right),\left(t, t^{\prime}\right)} Q\left(K\left(\tau_{1}^{\prime}, s^{\prime}\right)^{*}-K\left(\tau_{2}^{\prime}, s^{\prime}\right)^{*}\right)$,

and by the same techniques as above our goal is to obtain an analytic inequality as in (iv). Consider the approximating integral given by

$$
N_{\mathcal{P} \times \mathcal{P}^{\prime}}:=\sum_{\substack{[u, v] \in \mathcal{P} \\\left[u^{\prime}, v^{\prime}\right] \in \mathcal{P}^{\prime}}}\left(K\left(\tau_{1}, u\right)-K\left(\tau_{2}, u\right)\right) \square_{\left(u, u^{\prime}\right),\left(v, v^{\prime}\right)} Q\left(K\left(\tau_{1}^{\prime}, u^{\prime}\right)^{*}-K\left(\tau_{2}^{\prime}, u^{\prime}\right)^{*}\right) .
$$

Thus, we obtain the inequality in (iv) by following the exact same steps as for the existence with the integrand $K \square Q K^{*}$. However, instead of relying on the norm $\|K\|_{\eta, 3}$ as given in (3.15) to obtain our bounds, we need to use $\|K\|_{\eta, 4}$ given in (3.16) as this represents the regularity of the kernel over the rectangular increment (i.e. in both upper and lower variables). Indeed, when arriving at the step similar to (4.35), we set

$$
\Xi^{\tau_{1}, \tau_{2}, \tau_{1}^{\prime}, \tau_{2}^{\prime}}\left(u, u^{\prime}, v, v^{\prime}\right):=\left(K\left(\tau_{1}, u\right)-K\left(\tau_{2}, u\right)\right) \square_{\left(u, u^{\prime}\right),\left(v, v^{\prime}\right)} Q\left(K\left(\tau_{1}^{\prime}, u^{\prime}\right)^{*}-K\left(\tau_{2}^{\prime}, u^{\prime}\right)^{*}\right)
$$

and observe that

$$
\begin{aligned}
\delta_{z}^{1} \delta_{z^{\prime}}^{2} \Xi^{\tau_{1}, \tau_{2}, \tau_{1}^{\prime}, \tau_{2}^{\prime}}\left(u, u^{\prime}, v, v^{\prime}\right)=\left(K\left(\tau_{1}, u\right)-K\left(\tau_{2}, u\right)-K\left(\tau_{1}, z\right)+K\left(\tau_{2}, z\right)\right) \\
\times \square_{\left(z, z^{\prime}\right),\left(v, v^{\prime}\right)} Q\left(K\left(\tau_{1}^{\prime}, u^{\prime}\right)^{*}-K\left(\tau_{2}^{\prime}, u^{\prime}\right)^{*}-K\left(\tau_{1}^{\prime}, z^{\prime}\right)^{*}+K\left(\tau_{2}^{\prime}, z^{\prime}\right)^{*}\right) .
\end{aligned}
$$

We then need to bound this expression in a similar way as we did in (4.36). Using the quantity defined in (3.15), it is readily seen that for $\theta_{1}, \theta_{2} \in[0,1]$

$$
\left\|\delta_{z}^{1} \delta_{z^{\prime}}^{2} \Xi^{\tau_{1}, \tau_{2}, \tau_{1}^{\prime}, \tau_{2}^{\prime}}\left(u, u^{\prime}, v, v^{\prime}\right)\right\|_{\mathrm{op}} \lesssim\|K\|_{\eta, 4}^{2}\|Q\|_{\alpha,(1,1)}
$$


Infinite dimensional pathwise Volterra processes

$$
\times\left[\left|\tau_{1}-\tau_{2}\right|\left|\tau_{1}^{\prime}-\tau_{2}^{\prime}\right|\right]^{\theta_{1}}\left[\left|\tau_{2}-v\right|\left|\tau_{2}^{\prime}-v^{\prime}\right|\right]^{-\theta_{1}-\theta_{2}-\eta} \mid\left[|v-u|\left|u^{\prime}-v^{\prime}\right|\right]^{\alpha+\theta_{2}} .
$$

We then observe that for a parameter $\zeta \in[0,1]$ we have

$$
\left|\tau_{2}-v\right|^{-\theta_{1}-\theta_{2}-\eta} \leq\left|\tau_{2}-t\right|^{-\theta_{1}+\zeta}\left|\tau_{2}-v\right|^{-\zeta-\theta_{2}-\eta}
$$

and similarly for the parameters $\left(\tau_{2}^{\prime}, t^{\prime}, v^{\prime}\right) \in \Delta_{3}^{T}$. it follows that

$$
\begin{aligned}
& \left\|\delta_{z}^{1} \delta_{z^{\prime}}^{2} \Xi^{\tau_{1}, \tau_{2}, \tau_{1}^{\prime}, \tau_{2}^{\prime}}\left(u, u^{\prime}, v, v^{\prime}\right)\right\|_{\mathrm{op}} \lesssim\|K\|_{\eta, 4}^{2}\|Q\|_{\alpha,(1,1)} \\
\times & {\left[\left|\tau_{1}-\tau_{2} \| \tau_{1}^{\prime}-\tau_{2}^{\prime}\right|\right]^{\theta_{1}}\left[\left|\tau_{2}-t\right|\left|\tau_{2}^{\prime}-t^{\prime}\right|\right]^{-\theta_{1}+\zeta}\left[\left|\tau_{2}-v \| \tau_{2}^{\prime}-v^{\prime}\right|\right]^{-\theta_{2}-\eta-\zeta} \mid\left[\left|v-u \| u^{\prime}-v^{\prime}\right|\right]^{\alpha+\theta_{2}} . }
\end{aligned}
$$

Note that we are not integrating over the variables $\left(\tau_{1}, \tau_{2}, t\right),\left(\tau_{1}^{\prime}, \tau_{2}^{\prime}, t^{\prime}\right) \in \Delta_{3}^{T}$, and these will therefore not affect the sewing arguments in (4.39) and below. We now choose $\theta_{1}, \theta_{2}, \zeta \in[0,1]$ in the following way: $\beta=\alpha+\theta_{2}>1, \kappa:=\eta+\theta_{2}+\zeta<1$. Since $\rho=\alpha-\eta>0$ we can choose $\zeta \in[0, \rho)$. Then one can simply check that there exists a $\theta_{2} \in[0,1]$ such that $\beta>1$ and $\kappa<1$. By following the steps from (4.36) and below, one can conclude that (iv) holds. This completes the proof.

Remark 18. We point out that the integral $\int_{0}^{t} \int_{0}^{t^{\prime}} K(\tau, r) d^{2} Q\left(r, r^{\prime}\right) K\left(\tau^{\prime}, r^{\prime}\right)^{*}$ is linear in $Q$, and bilinear in $K$. By this we mean that for $Q, \tilde{Q} \in \mathcal{Q}_{\alpha}$

$$
\begin{aligned}
\int_{0}^{t} \int_{0}^{t^{\prime}} & K(\tau, r) d^{2}[Q+\tilde{Q}]\left(r, r^{\prime}\right) K\left(\tau^{\prime}, r^{\prime}\right)^{*} \\
& =\int_{0}^{t} \int_{0}^{t^{\prime}} K(\tau, r) d^{2} Q\left(r, r^{\prime}\right) K\left(\tau^{\prime}, r^{\prime}\right)^{*}+\int_{0}^{t} \int_{0}^{t^{\prime}} K(\tau, r) d^{2} \tilde{Q}\left(r, r^{\prime}\right) K\left(\tau^{\prime}, r^{\prime}\right)^{*}
\end{aligned}
$$

and similarly for the bilinearity with respect to $K$. This follows directly from the construction of the integral as a limit of Riemann sums, and a simple verification can be done by going through the proof above using the integrand $K(\tau, u) \square_{\left(u, u^{\prime}\right),\left(v, v^{\prime}\right)}[Q+$ $\tilde{Q}] K\left(\tau^{\prime}, r^{\prime}\right)^{*}$. For conciseness we omit a more detailed proof here.

Remark 19. From the derivations in (4.5), a different notation for the integral

$$
\int_{0}^{t} \int_{0}^{t^{\prime}} K(\tau, r) d^{2} Q\left(r, r^{\prime}\right) K\left(\tau^{\prime}, r^{\prime}\right)^{*}
$$

could be used. By the expression

$$
\int_{0}^{t} K(\tau, r) \int_{0}^{t^{\prime}} Q\left(d r, d r^{\prime}\right) K\left(\tau^{\prime}, r^{\prime}\right)^{*}
$$

we mean the integration of $K\left(\tau^{\prime}, r^{\prime}\right)^{*}$ with respect to $Q\left(r, d r^{\prime}\right)$ to form the integral $\int_{0}^{t^{\prime}} Q\left(r, d r^{\prime}\right) K\left(\tau^{\prime}, r^{\prime}\right)^{*}$, followed by the integration of $K(\tau, r)$ with respect to the integrand $\int_{0}^{t^{\prime}} Q\left(d r, d r^{\prime}\right) K\left(\tau^{\prime}, r^{\prime}\right)$.

A direct consequence of Theorem 17 is that the covariance functions constructed from $K \in \mathcal{K}_{\eta}$ and $Q \in \mathcal{Q}_{\alpha}$ is again a covariance function in $\mathcal{Q}_{\zeta}$ for any $\zeta \in[0, \alpha-\eta)$. We summarize this in the next Proposition.

Proposition 20. By restricting the domain of $\mathcal{I}$ to the square $[0, T]^{2}$ by considering the $\operatorname{map}\left(t, t^{\prime}\right) \mapsto \mathcal{I}^{t, t^{\prime}}(K, Q)\left(0,0, t, t^{\prime}\right)$, the integration map $\mathcal{I}$ is a continuous operator from $\mathcal{K}_{\eta} \times \mathcal{Q}_{\alpha}$ to $\mathcal{Q}_{\zeta}$ for any $\zeta \in[0, \alpha-\eta)$. Moreover, we have that

$$
\|\mathcal{I}(K, Q)\|_{\mathcal{Q}_{\zeta}} \leq C\|K\|_{\mathcal{K}_{\eta}}^{2}\|Q\|_{\mathcal{Q}_{\alpha}}
$$


Proof. This follows from a combination of the estimates in (i)-(iv) given in Theorem 17. We denote by $\Delta_{s, t} K(\cdot, r)$ the increment $K(t, r)-K(s, r)$. Observe that

$$
\begin{aligned}
& \square_{\left(s, s^{\prime}\right),\left(t, t^{\prime}\right)} \int_{0}^{\cdot} \int_{0}^{\cdot^{\prime}} K(\cdot, r) d^{2} Q\left(r, r^{\prime}\right) K\left(\cdot^{\prime}, r^{\prime}\right)^{*}=\int_{s}^{t} \int_{s^{\prime}}^{t^{\prime}} K(t, r) d^{2} Q\left(r, r^{\prime}\right) K\left(t^{\prime}, r^{\prime}\right)^{*} \\
& +\int_{0}^{s} \int_{s^{\prime}}^{t^{\prime}} \Delta_{s, t} K(\cdot, r) d^{2} Q\left(r, r^{\prime}\right) K\left(t^{\prime}, r^{\prime}\right)^{*}+\int_{s}^{t} \int_{0}^{s^{\prime}} K(t, r) d^{2} Q\left(r, r^{\prime}\right) \Delta_{s^{\prime}, t^{\prime}} K\left(\cdot^{\prime}, r^{\prime}\right)^{*} \\
& +\int_{0}^{s} \int_{0}^{s^{\prime}} \Delta_{s, t} K(\cdot, r) d^{2} Q\left(r, r^{\prime}\right) \Delta_{s^{\prime}, t^{\prime}} K\left(\cdot^{\prime}, r^{\prime}\right)^{*} .
\end{aligned}
$$

Our goal is to check that

$$
\left\|\square_{\left(s, s^{\prime}\right),\left(t, t^{\prime}\right)} \int_{0}^{.} \int_{0}^{.^{\prime}} K(\cdot, r) d^{2} Q\left(r, r^{\prime}\right) K\left(\cdot^{\prime}, r^{\prime}\right)^{*}\right\|_{o p} \lesssim\left[\left|t-s \| t^{\prime}-s^{\prime}\right|\right]^{\alpha-\eta},
$$

and thus, by verifying that each of the integrals on the right-hand side of (4.53) satisfies the above bound, we are done. Each of the four terms on the right hand side above corresponds to the inequalities in (i)-(iv) in Theorem 17 plus some one-dimensional integral terms which can be treated with the one-dimensional Volterra Sewing Lemma 10. We will illustrate this by considering the first term on the right hand side of the above equality (4.53). It is readily checked that by addition and subtraction of three terms

$$
\begin{array}{r}
\int_{s}^{t} \int_{s^{\prime}}^{t^{\prime}} K(t, s) d^{2} Q\left(r, r^{\prime}\right) K\left(t^{\prime}, r^{\prime}\right)^{*}, \int_{s}^{t} \int_{s^{\prime}}^{t^{\prime}} K(t, r) d^{2} Q\left(r, r^{\prime}\right) K\left(t^{\prime}, s^{\prime}\right)^{*}, \\
\text { and } K(t, s) \square\left(s, s^{\prime}\right),\left(t, t^{\prime}\right) Q\left(r, r^{\prime}\right) K\left(t^{\prime}, s^{\prime}\right)^{*},
\end{array}
$$

it follows that

$$
\begin{aligned}
\int_{s}^{t} \int_{s^{\prime}}^{t^{\prime}} & K(t, r) d^{2} Q\left(r, r^{\prime}\right) K\left(t^{\prime}, r^{\prime}\right)^{*} \\
& =\int_{s}^{t} \int_{s^{\prime}}^{t^{\prime}}[K(t, r)-K(t, s)] d^{2} Q\left(r, r^{\prime}\right)\left[K\left(t^{\prime}, r^{\prime}\right)-K\left(t^{\prime}, s^{\prime}\right)\right]^{*} \\
& +\int_{s}^{t} \int_{s^{\prime}}^{t^{\prime}} K(t, s) d^{2} Q\left(r, r^{\prime}\right) K\left(t^{\prime}, r^{\prime}\right)^{*} \\
& +\int_{s}^{t} \int_{s^{\prime}}^{t^{\prime}} K(t, r) d^{2} Q\left(r, r^{\prime}\right) K\left(t^{\prime}, s^{\prime}\right)^{*}-K(t, s) \square\left(s, s^{\prime}\right),\left(t, t^{\prime}\right) Q K\left(t^{\prime}, s^{\prime}\right) .
\end{aligned}
$$

We can bound the first integral expression on the right-hand side by application of (i) in Theorem 17. The two other integral terms are one-dimensional in the sense that we are only integrating one of the kernels $K$ in either $r$ or $r^{\prime}$. By the inequality obtained in (4.25) and (4.27), it follows that

$$
\begin{aligned}
\| \int_{s}^{t} \int_{s^{\prime}}^{t^{\prime}} K(t, s) d^{2} Q\left(r, r^{\prime}\right) K\left(t^{\prime}, r^{\prime}\right)^{*}-K(t, s) & \square_{\left(s, s^{\prime}\right),\left(t, t^{\prime}\right)} Q K\left(t^{\prime}, s^{\prime}\right) \|_{o p} \\
& \lesssim\|K\|_{\mathcal{K}_{\eta}}^{2}\|Q\|_{\alpha}|t-s|^{\alpha-\eta}\left|t^{\prime}-s^{\prime}\right|^{\alpha-\eta}
\end{aligned}
$$

and similarly we get

$$
\begin{aligned}
\| \int_{s}^{t} \int_{s^{\prime}}^{t^{\prime}} K(t, r) d^{2} Q\left(r, r^{\prime}\right) K\left(t^{\prime}, s^{\prime}\right)^{*}-K(t, s) & \square_{\left(s, s^{\prime}\right),\left(t, t^{\prime}\right)} Q K\left(t^{\prime}, s^{\prime}\right) \|_{o p} \\
& \lesssim\|K\|_{\mathcal{K}_{\eta}}^{2}\|Q\|_{\alpha}|t-s|^{\alpha-\eta}\left|t^{\prime}-s^{\prime}\right|^{\alpha-\eta} .
\end{aligned}
$$


At last, it is readily checked that also

$$
\left\|K(t, s) \square_{\left(s, s^{\prime}\right),\left(t, t^{\prime}\right)} Q K\left(t^{\prime}, s^{\prime}\right)\right\|_{o p} \lesssim\|K\|_{\mathcal{K}_{\eta}}^{2}\|Q\|_{\alpha}|t-s|^{\alpha-\eta}\left|t^{\prime}-s^{\prime}\right|^{\alpha-\eta} .
$$

Thus a combination of (4.55), (4.56) and (4.57) as well as the bound in (i) of Theorem 17, we obtain

$$
\left\|\int_{s}^{t} \int_{s^{\prime}}^{t^{\prime}} K(t, r) d^{2} Q\left(r, r^{\prime}\right) K\left(t^{\prime}, r^{\prime}\right)^{*}\right\|_{o p} \lesssim\|K\|_{\mathcal{K}_{\eta}}^{2}\|Q\|_{\alpha}|t-s|^{\alpha-\eta}\left|t^{\prime}-s^{\prime}\right|^{\alpha-\eta} .
$$

By a similar analysis, one obtains equivalent bounds for the three other integral terms on the right-hand side of (4.53) by appealing to (ii)-(iv) of Theorem 17 as well as bounds for one-dimensional integral terms treated (as done above) by application of Lemma 10. However, in this case, the bound will be with respect to any exponent $\zeta \in[0, \alpha-\gamma)$, as the inequalities in (ii)-(iv) satisfy this type of regularity condition. It therefore follows that the left-hand side of (4.53) satisfies

$$
\left\|\square_{\left(s, s^{\prime}\right),\left(t, t^{\prime}\right)} \int_{0}^{\cdot} \int_{0}^{.^{\prime}} K(\cdot, r) d^{2} Q\left(r, r^{\prime}\right) K\left(\cdot^{\prime}, r^{\prime}\right)^{*}\right\|_{o p} \lesssim\|K\|_{\mathcal{K}_{\eta}}^{2}\|Q\|_{\alpha}|t-s|^{\zeta}\left|t^{\prime}-s^{\prime}\right|^{\zeta},
$$

for any $\zeta \in[0, \alpha-\gamma)$. Since the $\operatorname{map}\left(t, t^{\prime}\right) \mapsto \int_{0}^{t} \int_{0}^{t^{\prime}} K(\cdot, r) d^{2} Q\left(r, r^{\prime}\right) K\left(\cdot^{\prime}, r^{\prime}\right)^{*}$ is zero on the boundary of $[0, T]^{2}$, we conclude by Remark 16 that the covariance operator is contained in $\mathcal{Q}_{\zeta}$ for any $\zeta \in[0, \alpha-\gamma)$.

Another consequence of the construction of the double Young-Volterra integral is stability estimates in terms of the driving covariance $Q$ and the Volterra kernel $K$. We summarize this in the following proposition.

Proposition 21. Let $K, \tilde{K} \in \mathcal{K}_{\eta}$, with $\eta \in(0,1)$. For a constant $\alpha \in(\eta, 1]$, assume that $Q$ and $\tilde{Q}$ are both $\alpha$-regular covariance functions in $\mathcal{Q}_{\alpha}$. Furthermore, let $M>0$ be a constant such that $\|K\|_{\mathcal{K}_{\eta}} \vee\|\tilde{K}\|_{\mathcal{K}_{\eta}} \vee\|\mathcal{Q}\|_{\mathcal{Q}_{\alpha}} \vee\|\tilde{\mathcal{Q}}\|_{\mathcal{Q}_{\alpha}} \leq M$. Then the following stability estimate holds for any $\zeta \in[0, \alpha-\gamma)$.

$$
\|\mathcal{I}(K, Q)-\mathcal{I}(\tilde{K}, \tilde{Q})\|_{\mathcal{Q}_{\zeta}} \leq C_{M}\left(\|K-\tilde{K}\|_{\mathcal{K}_{\eta}}+\|Q-\tilde{Q}\|_{\mathcal{Q}_{\alpha}}\right) .
$$

Proof. This follows directly from the proof of Theorem 17, and Proposition 20. First, it is readily checked that Theorem 17 may canonically be extended to integrals on the form

$$
\mathcal{I}(K, Q, L)\left(t, t^{\prime}\right):=\int_{0}^{t} \int_{0}^{t^{\prime}} K(t, r) d^{2} Q\left(r, r^{\prime}\right) L\left(t^{\prime}, r^{\prime}\right)^{*},
$$

where $K, L \in \mathcal{K}_{\eta}$ and $Q \in \mathcal{Q}_{\alpha}$. We therefore assume at this point that the above integral is well defined in the same way as shown in Theorem 17. This leads to an extension of inequality (4.52) given on the form

$$
\|\mathcal{I}(K, Q, L)\|_{\mathcal{Q}_{\zeta}} \leq C\|K\|_{\mathcal{K}_{\eta}}\|Q\|_{\mathcal{Q}_{\alpha}}\|L\|_{\mathcal{K}_{\eta}},
$$

for $\zeta \in[0, \alpha-\gamma)$. Observe that the difference $\mathcal{I}(K, Q)-\mathcal{I}(\tilde{K}, \tilde{Q})$ is equal to

$$
\mathcal{I}(K, Q)-\mathcal{I}(\tilde{K}, \tilde{Q})=D(K, \tilde{K})+D(Q, \tilde{Q})
$$

where we define

$$
D(K, \tilde{K}):=\mathcal{I}(K, Q)-\mathcal{I}(\tilde{K}, Q) \text { and } D(Q, \tilde{Q}):=\mathcal{I}(\tilde{K}, Q)-\mathcal{I}(\tilde{K}, \tilde{Q}) .
$$

Recall from Remark 18 that the integral operator is bilinear in $K$ and linear in $Q$. Moreover, since $K$ and $\tilde{K}$ are both linear operators on $H$, their difference is also a linear 
operator on $H$, and since $\mathcal{K}_{\eta}$ is a linear space, it follows that $K-\tilde{K} \in \mathcal{K}_{\eta}$. Similarly, $Q-\tilde{Q} \in \mathcal{Q}_{\alpha}$. This yields,

$$
\begin{aligned}
D(K, \tilde{K})\left(t, t^{\prime}\right)= & \int_{0}^{t} \int_{0}^{t^{\prime}} \tilde{K}(t, r) d^{2} Q\left(r, r^{\prime}\right)(K-\tilde{K})\left(t^{\prime}, r^{\prime}\right)^{*} \\
& +\int_{0}^{t} \int_{0}^{t^{\prime}}(K-\tilde{K})(t, r) d^{2} Q\left(r, r^{\prime}\right) K\left(t^{\prime}, r^{\prime}\right)^{*} \\
= & \mathcal{I}(\tilde{K}, Q, K-\tilde{K})\left(t, t^{\prime}\right)+\mathcal{I}(K-\tilde{K}, Q, K)\left(t, t^{\prime}\right) .
\end{aligned}
$$

Invoking the inequality (4.59) twice, we find

$$
\|D(K, \tilde{K})\|_{\mathcal{Q}_{\zeta}} \leq C_{M}\|K\|_{\mathcal{K}_{\eta}}\|K-\tilde{K}\|_{\mathcal{K}_{\eta}}\|Q\|_{\mathcal{Q}_{\alpha}}
$$

Through similar manipulations using that $Q-\tilde{Q} \in \mathcal{Q}_{\alpha}$ it is seen from Proposition 20 that $D(Q, \tilde{Q})$ can be bounded by

$$
\|D(Q, \tilde{Q})\|_{\mathcal{Q}_{\zeta}} \leq C_{M}\|K\|_{\mathcal{K}_{\eta}}\|Q-\tilde{Q}\|_{\mathcal{Q}_{\alpha}}
$$

We can now majorize the difference on the left hand side of (4.58) by using relation (4.60) and the triangle inequality, as well as the estimates in (4.61) and (4.62) to obtain

$$
\|\mathcal{I}(K, Q)-\mathcal{I}(\tilde{K}, \tilde{Q})\|_{\mathcal{Q}_{\zeta}} \leq C_{M}\left(\|K-\tilde{K}\|_{\mathcal{K}_{\eta}}+\|Q-\tilde{Q}\|_{\mathcal{Q}_{\alpha}}\right),
$$

which proves our claim.

The stability estimate in Proposition 21 tells us that the Volterra processes are Lipschitz continuous in both the kernel $K$ and the covariance functional $Q$ of the noise. Thus, small model errors or statistical estimation errors in the kernel $K$ and/or the covariance functional $Q$ lead to small errors in the resulting Volterra processes. This holds $\omega$-wise and is therefore a very strong stability in a probabilistic context.

\subsection{Characteristic functionals of Volterra processes driven by Gaussian noise}

An important question to ask is whether the pathwise Volterra process constructed in Proposition (14) is a Gaussian process when the driving noise $W$ is a Hilbert-valued Gaussian process. The next proposition gives an affirmative answer to this question.

Proposition 22. Consider a Hilbert-valued zero-mean Gaussian process $W:[0, T] \times \Omega \rightarrow$ $H$ with covariance operator $Q_{W}:[0, T]^{2} \rightarrow \mathcal{L}(H)$, and assume $t \mapsto W(t, \omega)$ is $\beta$-Hölder continuous with $\beta \in(0,1)$ for $\omega \in \mathcal{N}^{c} \in \mathcal{F}$, where $\mathcal{N}^{c}$ is of full measure. Let $K \in \mathcal{K}_{\eta}$ with $\zeta:=\beta-\eta>0$, and that the covariance operator $Q_{W} \in \mathcal{Q}_{\alpha}$ for $\rho=\alpha-\eta>0$. For any $\omega \in \mathcal{N}^{c}$, let $X^{\cdot}(\cdot, \omega)$ be given as the Volterra process

$$
X^{\tau}(t, \omega)=\int_{0}^{t} K(\tau, s) d W(s, \omega)
$$

where the integral is constructed as in Proposition 14. Then $(t, \omega) \mapsto X^{t}(t, \omega)$ is a Hilbert-valued zero-mean Gaussian process on the probability space $(\Omega, \mathcal{F}, \mathbb{P})$, and the characteristic functional of $X$ is given by

$$
\mathbb{E}\left[\exp \left(i\left\langle X^{\tau}(t), f\right\rangle\right)\right]=\exp \left(-\frac{1}{2}\left\langle\int_{0}^{t} \int_{0}^{t} K(\tau, r) d^{2} Q_{W}\left(r, r^{\prime}\right) K\left(\tau, r^{\prime}\right)^{*} f, f\right\rangle\right),
$$

for any $f \in H$. 
Proof. We begin to prove that for each $(\tau, t) \in \Delta_{2}^{T}, X^{\tau}(t, \cdot)$ is a Gaussian random variable. To this end, it is sufficient to prove that the characteristic functional of $X^{\tau}(t, \cdot)$ is that of a Gaussian, and that it is given by (4.64). Observe that by continuity of the exponential function and the construction of $X$ as the limit of a Riemann type sum as given in Proposition 14, we have

$$
\begin{aligned}
\mathbb{E}\left[\exp \left(i\left\langle\int_{0}^{t} K(\tau, s) d W(s, \omega), f\right\rangle\right)\right] & =\mathbb{E}\left[\lim _{|\mathcal{P}| \rightarrow 0} \exp \left(i \sum_{[u, v] \in \mathcal{P}}\langle K(\tau, u)(W(v)-W(u)), f\rangle\right)\right] \\
& =\mathbb{E}\left[\lim _{|\mathcal{P}| \rightarrow 0} \exp \left(i \sum_{[u, v] \in \mathcal{P}}\left\langle W(v)-W(u), K(\tau, u)^{*} f\right\rangle\right)\right] .
\end{aligned}
$$

Since the exponential $|\exp (i\langle g, f\rangle)| \leq 1$ for any $f, g \in H$, it follows from the dominated convergence theorem that

$$
\begin{aligned}
\mathbb{E}\left[\lim _{|\mathcal{P}| \rightarrow 0} \exp \left(i \sum_{[u, v] \in \mathcal{P}}\left\langle W(v)-W(u), K(\tau, u)^{*} f\right\rangle\right)\right] \\
=\lim _{|\mathcal{P}| \rightarrow 0} \mathbb{E}\left[\exp \left(i \sum_{[u, v] \in \mathcal{P}}\left\langle W(v)-W(u), K(\tau, u)^{*} f\right\rangle\right)\right] .
\end{aligned}
$$

Using that the sum $\sum_{[u, v] \in \mathcal{P}}\left\langle W(v)-W(u), K(\tau, u)^{*} f\right\rangle$ is Gaussian, since $W$ is a Gaussian process (see Def. 2), and by similar computations as given in (4.5) we obtain that the following identity holds

$$
\begin{aligned}
\mathbb{E}\left[\exp \left(i \sum_{[u, v] \in \mathcal{P}}\left\langle W(v)-W(u), K(\tau, u)^{*} f\right\rangle\right)\right] \\
=\exp \left(-\frac{1}{2} \sum_{\substack{[u, v] \in \mathcal{P} \\
\left[u^{\prime}, v^{\prime}\right] \in \mathcal{P}}}\left\langle\square_{\left(u, u^{\prime}\right),\left(v, v^{\prime}\right)} Q_{W} K\left(\tau, u^{\prime}\right)^{*} f, K(\tau, u)^{*} f\right\rangle\right) .
\end{aligned}
$$

By using the dual formulation of the operators again, and moving the double sum on this inside, we recognise that

$$
\begin{aligned}
\exp \left(-\frac{1}{2} \sum_{\substack{[u, v] \in \mathcal{P} \\
\left[u^{\prime}, v^{\prime}\right] \in \mathcal{P}}}\left\langle\square_{\left(u, u^{\prime}\right),\left(v, v^{\prime}\right)} Q_{W} K\left(\tau, u^{\prime}\right)^{*} f, K(\tau, u)^{*} f\right\rangle\right) \\
=\exp \left(-\frac{1}{2}\left\langle\sum_{\substack{[u, v] \in \mathcal{P} \\
\left[u^{\prime}, v^{\prime}\right] \in \mathcal{P}}} K(\tau, u) \square_{\left(u, u^{\prime}\right),\left(v, v^{\prime}\right)} Q_{W} K\left(\tau, u^{\prime}\right)^{*} f, f\right\rangle\right) .
\end{aligned}
$$

Taking limits as the partition goes to zero, we obtain exactly the operator-valued integral

$$
\begin{aligned}
\int_{0}^{t} \int_{0}^{t} K(\tau, r) d^{2} Q_{W}\left(r, r^{\prime}\right) K\left(\tau, r^{\prime}\right)^{*} & \\
& =\lim _{|\mathcal{P}| \rightarrow 0} \sum_{\substack{[u, v] \in \mathcal{P} \\
\left[u^{\prime}, v^{\prime}\right] \in \mathcal{P}}} K(\tau, u) \square_{\left(u, u^{\prime}\right),\left(v, v^{\prime}\right)} Q_{W} K\left(\tau, u^{\prime}\right)^{*} .
\end{aligned}
$$

By again recalling the derivations in (4.5), we have that

$$
\mathbb{E}\left[\left(X_{\mathcal{P}}^{\tau}(t)\right)^{\otimes 2}\right]=\sum_{\substack{[u, v] \in \mathcal{P} \\\left[u^{\prime}, v^{\prime}\right] \in \mathcal{P}}} K(\tau, u) \square_{\left(u, u^{\prime}\right),\left(v, v^{\prime}\right)} Q_{W} K\left(\tau, u^{\prime}\right)^{*}
$$


This shows that the right-hand side is symmetric and positive semi-definite operator, properties which are preserved after taking limits. Thus, the operator in (4.68) a bounded linear operator on $H$ which is symmetric and positive semi-definite. Combining our considerations and identities obtained in (4.68), (4.67), (4.66) and (4.65), we can see that

$$
\begin{aligned}
& \mathbb{E}\left[\exp \left(i\left\langle\int_{0}^{t} K(\tau, s) d W(s), f\right\rangle\right)\right] \\
&=\exp \left(-\frac{1}{2}\left\langle\int_{0}^{t} \int_{0}^{t} K(\tau, r) d^{2} Q_{W}\left(r, r^{\prime}\right) K\left(\tau, r^{\prime}\right)^{*} f, f\right\rangle\right) .
\end{aligned}
$$

Recognising that this is the characteristic functional of a Gaussian random variable in a Hilbert space with trace class covariance operator $Q_{X}^{\tau, \tau}(t, t) \in \mathcal{L}(H)$ given by

$$
Q_{X}^{\tau, \tau}(t, t)=\int_{0}^{t} \int_{0}^{t} K(\tau, r) d^{2} Q_{W}\left(r, r^{\prime}\right) K\left(\tau, r^{\prime}\right)^{*}
$$

proves that $X^{\tau}(t)=\int_{0}^{t} K(\tau, s) d W(s)$ is a Gaussian random variable in $H$ for each $(\tau, t) \in \Delta_{2}^{T}$.

In order to prove that $t \mapsto X(t)=X^{t}(t)=\int_{0}^{t} K(t, s) d W(s)$ is a Gaussian process, recall from Definition 2 that we need to show that for any $n \geq 1,\left\{t_{i}\right\}_{i=1}^{n} \subset[0, T]$, and $\left\{f_{i}\right\}_{i=1}^{n} \in H^{\times n},\left(\left\langle X\left(t_{1}\right), f_{1}\right\rangle, \ldots,\left\langle X\left(t_{n}\right), f_{n}\right\rangle\right)$ is an $n$-variate Gaussian random variable in $\mathbb{R}^{n}$. We prove this claim for $n=2$, and the case for $n \geq 2$ follows by by a similar argument, however being notationally much more involved.

For $t_{1}, t_{2} \in[0, T]$, we consider

$$
\left(\begin{array}{l}
\int_{0}^{t_{1}} K\left(t_{1}, r\right) d W(r) \\
\int_{0}^{t_{2}} K\left(t_{2}, r\right) d W(r)
\end{array}\right) \in H^{2}
$$

Define an operator $G:[0, T]^{4} \rightarrow \mathcal{L}\left(H^{2}\right)$ by

$$
G\left(t_{1}, t_{2}, u_{1}, u_{2}\right)=\left(\begin{array}{cc}
K\left(t_{1}, u_{1}\right) & 0 \\
0 & K\left(t_{2}, u_{2}\right)
\end{array}\right) .
$$

Both integrals in (4.71) are constructed as limits of Riemann type sums (as in Proposition 14 ) in the following way: Set $\mathcal{P}^{1}$ to be a partition over $\left[0, t_{1}\right]$ and $\mathcal{P}^{2}$ to be a partition over $\left[0, t_{2}\right]$, and we have that

$$
\begin{aligned}
& \left(\begin{array}{l}
\int_{0}^{t_{1}} K\left(t_{1}, r\right) d W(r) \\
\int_{0}^{t_{1}} K\left(t_{2}, r\right) d W(r)
\end{array}\right) \\
& \quad=\lim _{\left|\mathcal{P}^{1}\right| \rightarrow 0} \lim _{\left|\mathcal{P}^{2}\right| \rightarrow 0} \sum_{\left[u_{1}, v_{1}\right] \in \mathcal{P}^{1}} \sum_{\left[u_{2}, v_{2}\right] \in \mathcal{P}^{2}} G\left(t_{1}, t_{2}, u_{1}, u_{2}\right)\left(\begin{array}{l}
W\left(v_{1}\right)-W\left(u_{1}\right) \\
W\left(v_{2}\right)-W\left(u_{2}\right)
\end{array}\right)
\end{aligned}
$$

Set $F=\left(f_{1}, f_{2}\right) \in H^{2}, u=\left(u_{1}, u_{2}\right), v=\left(v_{1}, v_{2}\right), t=\left(t_{1}, t_{2}\right) \in[0, T]^{2}$, and define

$$
Z(v)-Z(u):=\left(\begin{array}{l}
W\left(v_{1}\right)-W\left(u_{1}\right) \\
W\left(v_{2}\right)-W\left(u_{2}\right)
\end{array}\right) .
$$

It is then readily checked that

$$
\begin{aligned}
\mathbb{E}[\langle G(t, u)(Z(v) & \left.-Z(u)), F\rangle_{H^{2}}\left\langle G\left(t, u^{\prime}\right)\left(Z\left(v^{\prime}\right)-Z\left(u^{\prime}\right)\right), F\right\rangle_{H^{2}}\right] \\
& =\mathbb{E}\left[\left\langle Z(v)-Z(u), G(t, u)^{*} F\right\rangle_{H^{2}}\left\langle Z\left(v^{\prime}\right)-Z\left(u^{\prime}\right), G\left(t, u^{\prime}\right)^{*} F\right\rangle_{H^{2}}\right],
\end{aligned}
$$


Infinite dimensional pathwise Volterra processes

and by similar computations as in (4.5) we obtain the following expression

$$
\begin{aligned}
\mathbb{E}\left[\langle G ( t , u ) ( Z ( v ) - Z ( u ) ) , F \rangle _ { H ^ { 2 } } \left\langleG ( t , u ^ { \prime } ) \left(Z\left(v^{\prime}\right)\right.\right.\right. & \left.\left.\left.-Z\left(u^{\prime}\right)\right), F\right\rangle_{H^{2}}\right] \\
& =\left\langle G(t, u) \square_{(u, v),\left(u^{\prime}, v^{\prime}\right)} Q_{Z} G\left(t, u^{\prime}\right)^{*} F, F\right\rangle_{H^{2}} .
\end{aligned}
$$

Let us first investigate the covariance $Q_{Z}$ associated to $Z$. By definition of $Z$, it follows that

$$
\begin{aligned}
& \square_{\left(u, u^{\prime}\right),\left(v, v^{\prime}\right)} Q_{Z} \\
& =\left(\begin{array}{ll}
\mathbb{E}\left[\left(W\left(v_{1}\right)-W\left(u_{1}\right)\right) \otimes\left(W\left(v_{1}^{\prime}\right)-W\left(u_{1}^{\prime}\right)\right)\right] & \mathbb{E}\left[\left(W\left(v_{1}\right)-W\left(u_{1}\right)\right) \otimes\left(W\left(v_{2}^{\prime}\right)-W\left(u_{2}^{\prime}\right)\right)\right] \\
\mathbb{E}\left[\left(W\left(v_{2}\right)-W\left(u_{2}\right)\right) \otimes\left(W\left(v_{1}^{\prime}\right)-W\left(u_{1}^{\prime}\right)\right)\right] & \mathbb{E}\left[\left(W\left(v_{2}\right)-W\left(u_{2}\right)\right) \otimes\left(W\left(v_{2}^{\prime}\right)-W\left(u_{2}^{\prime}\right)\right)\right]
\end{array}\right) \\
& =\left(\begin{array}{cc}
\square_{\left(u_{1}, u_{1}^{\prime}\right),\left(v_{1}, v_{1}^{\prime}\right)} Q_{W} & \square_{\left(u_{1}, u_{2}^{\prime}\right),\left(v_{1}, v_{2}^{\prime}\right)} Q_{W} \\
\square_{\left(u_{1}^{\prime}, u_{2}\right),\left(v_{1}^{\prime}, v_{2}\right)} Q_{W} & \square_{\left(u_{2}, u_{2}^{\prime}\right),\left(v_{2}, v_{2}^{\prime}\right)} Q_{W}
\end{array}\right) .
\end{aligned}
$$

The above expression for the covariance leads to the following expression for the appropriate composition of operators

$$
\begin{aligned}
& G(t, u) \square_{\left(u, u^{\prime}\right),\left(v, v^{\prime}\right)} Q_{Z} G\left(t, u^{\prime}\right)^{*} \\
& \quad=\left(\begin{array}{ll}
K\left(t_{1}, u_{1}\right) \square_{\left(u_{1}, u_{1}^{\prime}\right),\left(v_{1}, v_{1}^{\prime}\right)} Q_{W} K\left(t_{1}, u_{1}^{\prime}\right)^{*} & K\left(t_{1}, u_{1}\right) \square_{\left(u_{1}, u_{2}^{\prime}\right),\left(v_{1}, v_{2}^{\prime}\right)} Q_{W} K\left(t_{2}, u_{2}^{\prime}\right)^{*} \\
K\left(t_{2}, u_{2}\right) \square_{\left(u_{1}^{\prime}, u_{2}\right),\left(v_{1}^{\prime}, v_{2}\right)} Q_{W} K\left(t_{1}, u_{1}^{\prime}\right)^{*} & K\left(t_{2}, u_{2}\right) \square_{\left(u_{2}, u_{2}^{\prime}\right),\left(v_{2}, v_{2}^{\prime}\right)} Q_{W} K\left(t_{2}, u_{2}^{\prime}\right)^{*}
\end{array}\right) .
\end{aligned}
$$

The key observation here is that each of the elements in the above matrix only depends on four variables (in addition to $t_{1}$ and $t_{2}$ ). With this expression at hand, let $\mathcal{P}:=\mathcal{P}^{1} \times \mathcal{P}^{2}$ and $\mathcal{P}^{\prime}:=\mathcal{P}^{\prime, 1} \times \mathcal{P}^{\prime, 2}$ be two partitions of the rectangle $\left[0, t_{1}\right] \times\left[0, t_{2}\right]$. In particular, for $[u, v]=\left[u_{1}, v_{1}\right] \times\left[u_{2}, v_{2}\right] \in \mathcal{P},\left[u_{1}, v_{1}\right] \in \mathcal{P}^{1}$ and $\left[u_{2}, v_{2}\right] \in \mathcal{P}^{2}$. For notational ease define $\sum_{\mathcal{P}^{i} \times \mathcal{P}^{j}}:=\sum_{\left[u_{i}, v_{i}\right] \in \mathcal{P}^{i}} \sum_{\left[u_{j}, v_{j}\right] \in \mathcal{P}^{j}}$ for $i, j=1,2$. We then have that

$$
\begin{aligned}
& \sum_{[u, v] \in \mathcal{P}} \sum_{\left[u^{\prime}, v^{\prime}\right] \in \mathcal{P}^{\prime}} G(t, u) \square_{(u, v),\left(u^{\prime}, v^{\prime}\right)} Q_{Z} G\left(t, u^{\prime}\right)^{*} \\
= & \left(\begin{array}{lll}
\sum_{\mathcal{P}^{1} \times \mathcal{P}^{\prime}, 1} K\left(t_{1}, u_{1}\right) \square_{\left(u_{1}, u_{1}^{\prime}\right),\left(v_{1}, v_{1}^{\prime}\right)} Q_{W} K\left(t_{1}, u_{1}^{\prime}\right)^{*} & \sum_{\mathcal{P}^{1} \times \mathcal{P}^{\prime}, 2} K\left(t_{1}, u_{1}\right) \square_{\left(u_{1}, u_{2}^{\prime}\right),\left(v_{1}, v_{2}^{\prime}\right)} Q_{W} K\left(t_{2}, u_{2}^{\prime}\right)^{*} \\
\sum_{\mathcal{P}^{2} \times \mathcal{P}^{\prime}, 1} K\left(t_{2}, u_{2}\right) \square_{\left(u_{1}^{\prime}, u_{2}\right),\left(v_{1}^{\prime}, v_{2}\right)} Q_{W} K\left(t_{1}, u_{1}^{\prime}\right)^{*} & \sum_{\mathcal{P}^{2} \times \mathcal{P}^{\prime}, 2} K\left(t_{2}, u_{2}\right) \square_{\left(u_{2}, u_{2}^{\prime}\right),\left(v_{2}, v_{2}^{\prime}\right)} Q_{W} K\left(t_{2}, u_{2}^{\prime}\right)^{*}
\end{array}\right)
\end{aligned}
$$

On the right-hand side we obtain four double-sums approximating different covariance operators, as constructed in Theorem 17. In particular we have that for $i, j=1,2$

$$
\lim _{\substack{\left|\mathcal{P}^{i}\right| \rightarrow 0 \\\left|\mathcal{P}^{j}\right| \rightarrow 0}} \sum_{\mathcal{P}^{i} \times \mathcal{P}^{j}} K\left(t_{i}, u_{i}\right) \square_{\left(u_{i}, u_{j}\right),\left(v_{i}, v_{j}\right)} Q_{W} K\left(t_{j}, u_{j}\right)^{*}=\int_{0}^{t_{i}} \int_{0}^{t_{j}} K\left(t_{i}, r\right) d^{2} Q_{W}\left(r, r^{\prime}\right) K\left(t_{j}, r^{\prime}\right)^{*}
$$

from which we conclude that also the following expression is well-defined as a linear operator on $H^{2}$

$$
\lim _{\substack{\mathcal{P}|\rightarrow 0\\| \mathcal{P}^{\prime} \mid \rightarrow 0}} \sum_{\substack{u, v] \in \mathcal{P} \\ \sum_{\left[u^{\prime}, v^{\prime}\right] \in \mathcal{P}^{\prime}}}} G(t, u) \square_{u, v, u^{\prime}, v^{\prime}} Q_{Z} G\left(t, u^{\prime}\right)^{*}=\int_{0}^{t} \int_{0}^{t} G(t, s) d^{2} Q_{Z}\left(s, s^{\prime}\right) G\left(t, s^{\prime}\right)^{*},
$$

where $|\mathcal{P}|=\left|\mathcal{P}^{1}\right| \vee\left|\mathcal{P}^{2}\right|$, and similarly for $\mathcal{P}^{\prime}$. With all these tools at hand, we follow along the same lines of arguments leading to the proof that $\int_{0}^{t} K(t, s) d W(s)$ is a Gaussian random variable on $H$ as done in the first part of this proof, to see that

$$
\begin{aligned}
& \mathbb{E}\left[\exp \left(i\left\langle\left(\begin{array}{l}
\int_{0}^{t_{1}} K\left(t_{1}, r\right) d W(r) \\
\int_{0}^{t_{2}} K\left(t_{2}, r\right) d W(r)
\end{array}\right), F\right\rangle_{H^{2}}\right)\right] \\
& =\exp \left(-\frac{1}{2}\left\langle\int_{0}^{t} \int_{0}^{t} G(t, s) d^{2} Q_{Z}\left(s, s^{\prime}\right) G\left(t, s^{\prime}\right)^{*} F, F\right\rangle_{H^{2}}\right),
\end{aligned}
$$

where $\int_{0}^{t}=\int_{0}^{t_{1}} \int_{0}^{t_{2}}$. From this it follows that $\left(\begin{array}{l}\int_{0}^{t_{1}} K\left(t_{1}, r\right) d W(r) \\ \int_{0}^{t_{2}} K\left(t_{2}, r\right) d W(r)\end{array}\right)$ is a Gaussian random variable on $H^{2}$. A similar argument can be extended to any collection of times $t_{1}, \ldots, t_{n} \in[0, T]$, and thus we conclude that $t \mapsto \int_{0}^{t} K(t, r) d W(r)$ is a Gaussian process.

We remark in passing that the proof of Proposition 22 shows more than only the covariance operator of $X^{\tau}(t)$. Indeed, the proof provides (by inductive arguments) the covariance operator associated with the $H^{n}$-valued random variable $\left(X\left(t_{1}\right), \ldots, X\left(t_{n}\right)\right)$ for any sequence of times $\left\{t_{i}\right\}_{i=1}^{n} \subset[0, T]^{n}$, where $X(t):=X^{t}(t)$. 


\section{Applications}

In this Section we have collected some possible applications of our results on Gaussian Volterra processes in Hilbert space and the corresponding covariance functionals.

\subsection{Iterated stochastic process and their covariance operators}

Iterated stochastic processes has received much attention (e.g. [24, 9, 10, 30]). In [10], the authors propose to model a diffusion in a crack by iterated Brownian motions. In particular, one considers two independent Brownian motions $B^{i}:[0, T] \times \Omega_{i} \rightarrow \mathbb{R}^{n}$ for $i=1,2$, and then studies properties of the process $B^{1}\left(\left|B^{2}(t)\right|\right)$. We refer to $B^{1}$ as the state process and $B^{2}$ as the time process. Several interesting probabilistic and analytic properties can be obtained from these processes, see in particular [9] for a study of the pathwise properties of these processes, and [24] for relations with higher order fractional parabolic PDEs. A natural extension would be to consider infinite dimensional Gaussian processes indexed by irregular paths. The advantage of this pathwise approach is that the time process and the state process does not need to be independent. By this we mean that we fix an $\omega_{2} \in \Omega_{2}$, such that $t \mapsto B^{2}\left(t, \omega_{2}\right)$ is a continuous path, and one look at the conditional process $\mathbb{B}(t)=B^{1}\left(\left|B^{2}\left(\omega_{2}, t\right)\right|\right)$ as a random variable. This process is then a Gaussian process, and its covariance function is given by the composition of the covariance function of $B^{1}$ with the path $\left|B^{2}\left(\omega_{2}, t\right)\right|$. Due to the fact that $t \mapsto B^{2}\left(\omega_{2}, t\right)$ is Hölder continuous of order $\alpha<\frac{1}{2}$, it follows that the regularity of the covariance function is reduced accordingly. More generally, one can study infinite dimensional Gaussian processes with irregular time shifts. Let $I \subset \mathbb{R}_{+}, \alpha \in(0,1)$, and suppose $X:[0, T] \rightarrow I$, is a nowhere differentiable path, which is $\alpha$-Hölder continuous. Let $W: I \times \Omega \rightarrow H$ be a Gaussian process with an $\gamma$-regular covariance function $Q_{W}: I \times I \rightarrow \mathcal{L}(H)$ (according to Definition 15). Then the composition $W \circ X:[0, T] \rightarrow H$ is a Gaussian process, with covariance function

$$
Q_{W} \circ X(t, s)(f, g)=\mathbb{E}[\langle W \circ X(t), f\rangle\langle W \circ X(s), g\rangle]=Q_{W}(X(t), X(s))\langle f, g\rangle .
$$

It follows that the covariance $Q_{W} \circ X$ is $\alpha \gamma$-regular with $\alpha \gamma \in(0,1)$. Furthermore, one can study the Volterra process $Y(t)=\int_{0}^{t} K(t, r) d(W \circ X)(r)$, in order to introduce memory in the iterated process. Then Proposition 22 tells us that $Y$ is again Gaussian, given that the singularity of $K$ is integrable with respect to the regularity of the covariance function $Q_{W}$. In fact, since the covariance operator $Q_{W} \circ X$ is only Hölder continuous, the covariance operator needs to be constructed in terms Theorem 17, in order to make sense of this integral. This is of course due to the fact that $Q_{W} \circ X(t, s)$ is nowhere differentiable in a Frèchet sense, and thus classical constructions of the covariance functions of Gaussian Volterra processes (for example given in [19]) are not applicable.

\subsection{Construction of the rough path lift of Gaussian processes with irregular covariance functions}

At the core of rough paths lies a solution theory for controlled differential equations on the form

$$
d Y(t)=f(Y(t)) d X(t), \quad Y(0)=y \in H,
$$

where $f$ is sufficiently regular function and $X$ is an $\alpha$-Hölder continuous signal with $\alpha \in(0,1)$. If $\frac{1}{3}<\alpha \leq \frac{1}{2}$, one needs to lift the signal $X$ into a tuple $(X, \mathbb{X})$, where $\mathbb{X}:[0, T]^{2} \rightarrow H \otimes H$ represents the iterated integral of $X$. This tuple is then called the rough path corresponding to $X$. In fact, one requires the following two conditions to hold for $X$ and $\mathbb{X}$ for $s \leq u \leq t$,

$$
\mathbb{X}(s, t)-\mathbb{X}(s, u)-\mathbb{X}(u, t)=(X(u)-X(s)) \otimes(X(t)-X(u)),
$$


and

$$
\sup _{t \neq s \in[0, T]} \frac{|X(t)-X(s)|_{H}}{|t-s|^{\alpha}}<\infty \quad \text { and } \quad \sup _{t \neq s \in[0, T]} \frac{|\mathbb{X}(s, t)|_{H}}{|t-s|^{2 \alpha}}<\infty .
$$

Therefore, much attention is given to construct an object $\mathbb{X}$ which satisfies the above conditions for a given path $X$. In [14, Sec. 10.2], the construction of this object corresponding to a Gaussian noise is shown under a sufficient smoothness condition on the covariance function. This smoothness condition is stated in terms of two-dimensional $p$-variation norms, which can be seen to be equivalent to the Hölder continuity of the covariance operators introduced in Definition 15 under the assumption of continuity on the $p$-variation functions

In particular, in order to construct a "geometric" version of $\mathbb{X}$ when $X$ is a centred Gaussian process, [14, Thm. 10.4] tells us that it is sufficient that the covariance operator $Q_{X}$ is contained in $\mathcal{Q}_{\gamma}$ with $\gamma>\frac{1}{2}{ }^{2}$. Thus, the construction of covariance operators and their corresponding regularity provided in Theorem 17 and Proposition 20, open up for the construction of a rough path for Volterra processes driven by Gaussian paths with nowhere differentiable covariance operators. Such processes are, for example, illustrated in the above subsection by the class of iterated processes.

\subsection{Fractional Ornstein-Uhlenbeck process driven by irregular paths}

Fractional differential equations (FDEs) provide an alternative to classical ODEs, by introducing memory in the evolution of the process. This results in a non-local equation with interesting applications to several physical and social systems (e.g. [29, 13, 28])

Our concern here is an $H$-valued fractional Ornstein-Uhlenbeck stochastic differential equation on a given time interval $[0, T]$. Consider two parameters $(\alpha, \gamma) \in \mathbb{R}_{+} \times(0,1)$ with the relation $\alpha+\gamma-1>0$, and consider the equation formally given by

$$
D^{\alpha}(Y-y)(t)=A Y(t)+\dot{W}(t) .
$$

Here, $y \in H, A \in \mathcal{L}(H), W \in \mathcal{C}^{\gamma}([0, T], H)$ and $D^{\alpha}$ is the fractional time-derivative of order $\alpha$, given as in Definition 30 in the Appendix. The object $\dot{W}$ is interpreted only formally and is corresponding to the time-derivative $\frac{d}{d t} W(t)$. Since $W$ is only Hölder continuous, the derivative $\frac{d}{d t} W(t)$ does not exist, and thus we rather consider an integrated version of (5.1). With $I^{\alpha}$ being the fractional integral operator (see Definition 30 in the appendix), let us denote by $X=I^{\alpha}(\dot{W})$ which we interpret as the integral

$$
X(t)=\int_{0}^{t}(t-s)^{\alpha-1} d W(s)
$$

This integral is understood in the sense of Proposition 14 with $K(t, s)=(t-s)^{\alpha-1} I$ and $I \in \mathcal{L}(H)$ being the identity operator on $H$. The integral exists due to the assumption that $\alpha+\gamma-1>0$. Applying the fractional integral operator $I^{\alpha}$ on both sides of (5.1), we obtain the equation

$$
Y(t)=y+I^{\alpha}(A Y)(t)+X(t), \quad t \in[0, T] .
$$

We will need a few extra tools to be able to obtain an explicit representation of its solution, as well as the associated covariance operator in the Gaussian case.

First we present a version of Fubini's theorem, showing that we can exchange the order of integration of double integrals involving Riemann integration and Volterra-Young

\footnotetext{
${ }^{2}$ The condition is actually stated in terms of a two-dimensional $\rho$-variation norm for the covariance function, with $\rho \in[1,2)$. It is readily checked that the Hölder norms in Definition 15 are equivalent to this variation norm, under the assumption of continuity.
} 
integration. This property, that may be interesting in itself, is a crucial tool in proving a specific analytic representation of the fractional Ornstein-Uhlenbeck process in (5.3). As a corollary to our Fubini theorem, we show that the order of the fractional integral operator and a Young-Volterra integral can be interchanged. For conciseness, all proofs in this section are relegated to Appendix B.

Proposition 23. For $\gamma, \eta>0$ with $\rho:=\gamma-\eta>0$, let $Z:[0, T] \rightarrow H$ be given as

$$
Z(t)=\int_{0}^{t} K(t, s) d W(s)
$$

for $K \in \mathcal{K}_{\eta}$ and $W \in \mathcal{C}^{\gamma}([0, T], H)$, with the integral being defined as in Proposition 14 . Assume $G: \Delta_{2}^{T} \rightarrow \mathcal{L}(H)$ is in $\mathcal{K}_{\kappa}$ for some $\kappa \in(0,1)$. Then the following equality holds

$$
\int_{0}^{t} G(t, s) Z(s) d s=\int_{0}^{t} \int_{s}^{t} G(t, r) K(r, s) d r d W(s),
$$

where the integral on the right-hand side is again interpreted in terms of Lemma 10 with $\Xi^{\tau}(t, s):=\int_{s}^{\tau} G(\tau, r) K(r, s) d r(W(t)-W(s))$.

As already indicated, we apply the Fubini theorem to the fractional integral operator, and as we see in ther next Corollary, we can further establish a connection to MittagLeffler functions.

Corollary 24. Let $0<\alpha<\gamma<1$ and $\alpha+\gamma-1>0$ and $W \in \mathcal{C}^{\gamma}([0, T], H)$. Let furthermore $X$ be defined as in Proposition 14, with $K(t, s)=\frac{1}{\Gamma(1-\eta)}(t-s)^{-\eta}$ and $\gamma-\eta>0$. In particular, $X$ is given as the Volterra integral

$$
X(t)=\Gamma(1-\eta)^{-1} \int_{0}^{t}(t-s)^{-\eta} d W(s) .
$$

Then, for $A \in \mathcal{L}(H)$, the following relation holds

$$
\sum_{i=0}^{\infty} A^{\circ i} I^{i \alpha}(X)(t)=\int_{0}^{t}(t-s)^{-\eta} E_{\alpha, 1-\eta}\left(A(t-s)^{\alpha}\right) d W(s),
$$

where $E_{\alpha, \beta}(A t):=\sum_{i=0}^{\infty} \frac{A^{\circ i} t^{i}}{\Gamma(\alpha i+\beta)}$ for $t \in[0, T]$ is called the Mittag-Leffler operator, and the integrals are interpreted in sense of Proposition 14. Indeed, since $x \mapsto E_{\alpha, \beta}\left(x^{\alpha}\right)$ is smooth everywhere except at 0 where it is $\alpha$-Hölder continuous, we interpret the right-hand side of (5.5) using Proposition 14 with $K(t, s)=(t-s)^{-\eta} E_{\alpha, 1-\eta}\left(A(t-s)^{\alpha}\right)$.

Remark 25. The fact that the Mittag-Leffler operator is a bounded linear operator on $H$ is readily checked: for any $f \in H$ we have by the triangle inequality that

$$
\left|E_{\alpha, \beta}(A t) f\right|_{H}=\left|\sum_{i=0}^{\infty} \frac{A^{\circ i} t^{i} f}{\Gamma(\alpha i+\beta)}\right|_{H} \leq \sum_{i=0}^{\infty} \frac{\left|A^{\circ i} t^{i} f\right|_{H}}{\Gamma(\alpha i+\beta)} \leq|f|_{H} E_{\alpha, \beta}\left(\|A\|_{o p} t\right),
$$

where, in the last inequality, we have used that for a bounded linear operator $A$ and for any $i \geq 0$ we have $\left|A^{\circ i} f\right|_{H} \leq\|A\|_{o p}^{i}|f|_{H}$. The expression $E_{\alpha, \beta}\left(\|A\|_{o p} t\right)$ appearing on the right-hand side of (5.6) is the classical Mittag-Leffler function evaluated at $\|A\|_{o p} t$.

Theorem 26. For some $\gamma \in(0,1)$, let $W \in \mathcal{C}^{\gamma}([0, T], H)$, and assume that $A \in \mathcal{L}(H)$. For any $\alpha>1-\gamma$ let $X=I^{\alpha}(W)$ as given in (5.2), and assume $y \in H$. Then there exists a unique solution $Y \in \mathcal{C}^{\rho}([0, T], H)$ with $\rho<\gamma+\alpha-1$ to the equation

$$
Y(t)=y+A I^{\alpha}(Y)(t)+X(t) .
$$


Infinite dimensional pathwise Volterra processes

Moreover, the solution satisfies the following analytic formula

$$
Y(t)=E_{\alpha, 1}\left(A t^{\alpha}\right) y+\int_{0}^{t}(t-s)^{\alpha-1} E_{\alpha, \alpha}\left(A(t-s)^{\alpha}\right) d W(s),
$$

where the integral on the right-hand side of (5.8) is interpreted in sense of Corollary 24.

We observe from our analysis in Section 2 and 4, that $Y$ is a Gaussian process. In the next Corollary we apply Theorem 17 to state the covariance operator of $Y$.

Corollary 27. Consider parameters $\gamma, \alpha \in(0,1)$ such that $\rho=\gamma+\alpha-1>0$ and $\beta>0$ such that $\beta+\alpha-1>0$. Let $W$ be a Gaussian process in $\mathcal{C}^{\gamma}([0, T], H)$, with covariance operator $Q_{W} \in \mathcal{Q}_{\beta}$, and suppose $Y \in \mathcal{C}^{\rho}([0, T], H)$ is the solution to the fractional Ornstein-Uhlenbeck process given in Theorem 26 driven by $W$ with linear operator $A \in \mathcal{L}(H)$. Then the covariance operator associated to $Y$ is given by

$$
Q_{Y}\left(t, t^{\prime}\right)=\int_{0}^{t} \int_{0}^{t^{\prime}}(t-r)^{\alpha-1} E_{\alpha, \alpha}\left(A(t-r)^{\alpha}\right) d^{2} Q_{W}\left(r, r^{\prime}\right)\left(t^{\prime}-r^{\prime}\right)^{\alpha-1} E_{\alpha, \alpha}\left(A^{*}\left(t^{\prime}-r^{\prime}\right)^{\alpha}\right) .
$$

and $Q_{Y} \in \mathcal{Q}_{\eta}$ for any $\eta<\beta+\alpha-1$.

Remark 28. Observe that the regularity of $Y$ constructed as the Volterra process in (5.8) is of order $0<\rho<\gamma+\alpha-1$, where $\gamma$ is the regularity of $W$, However the regularity of the covariance $Q_{Y}$ is of order $\eta<\beta+\alpha-1$ where $\beta$ is the regularity of the covariance $Q_{W}$. A-priori, there is no imposed relationship between $\beta$ and $\gamma$, although in typical examples they will be strongly related (if not the same, see Example 3.1 for the case of fractional Brownian motion). On the other hand, given that we know the regularity of the covariance operator $Q_{W}$, then through Kolmogorov's continuity theorem 3, one can deduce the regularity of $W$ (which then relates $\gamma$ to $\beta$ ). However, this theorem is not an if and only if statement, and thus given that a stochastic process is $\gamma$-Hölder continuous, it is not obvious what regularity its covariance might have.

\subsection{Rough stochastic volatility models}

In this subsection we discuss various infinite dimensional extensions of rough stochastic volatility models that have attracted interest in recent years. Our starting point is the fractional Ornstein-Uhlenbeck process $Y$ defined in (5.8), where we for simplicity assume $y=0$.

Consider first a state space $H=\mathbb{R}$, and let the risk-neutral stock price dynamics with stochastic volatility be

$$
\frac{d S(t)}{S(t)}=\sigma(t, Y(t)) d B(t)
$$

for some Brownian motion $B$, possibly correlated with $W$, and where we suppose the risk-free interest rate to be zero. Recall that $W$ is the Gaussian process driving the fractional Ornstein-Uhlenbeck dynamics of $Y$. For example, choosing $\sigma(t, y)=\exp (y)$ would give a rough stochastic volatility model extending the class of models proposed by Gatheral, Jaisson and Rosenbaum [15]. In their paper, an Ornstein-Uhlenbeck process driven by a fractional Brownian motion is shown to provide an excellent fit the volatility of stock prices. We extend this class of models to allow for a fractional time-derivative in the dynamics as well, opening for further flexibility in the modelling. Furthermore, we can define a simple rough Heston model as the variance process

$$
V(t):=Y^{2}(t)
$$


or, more generally, taking $n$ independent copies of $\mathbb{R}$-valued processes $W_{i}, i=1, \ldots, n$ driving $Y_{i}(t)$ as in (5.8),

$$
V(t)=\sum_{i=1}^{n} Y_{i}^{2}(t)
$$

Choosing $\sigma(t, v)=\sqrt{v}$ would give a rough Heston stochastic volatility, providing a possible extension of the class of models considered by El Euch and Rosenbaum [13].

Let us return to a general separable Hilbert space $H$. Forward and futures prices can be realized as infinite dimensional stochastic processes, which call for operator-valued stochastic volatility models (see Benth, Rüdiger and Süss [7] and Benth and Krühner [6]). To this end, let $H$ be the state space of the forward curves, given by some separable Hilbert space of real-valued functions on $\mathbb{R}_{+}$. We restrict to $\mathbb{R}_{+}$as this plays the role of the time to maturity. A possible (simplistic) model for the risk-neutral forward price at time $t \geq 0$ is defined as

$$
d f(t)=\partial_{x} f(t) d t+\Sigma(t) d B(t)
$$

where $B$ is some $H$-valued Wiener process with covariance operator $Q_{B}$. A direct extension of the rough stochastic volatility model could be the following: supposing that $H$ is a Banach algebra, we define

$$
\Sigma(t):=\exp (Y(t))
$$

From the assumed algebra-structure of $H$, we can conclude that $\Sigma(t)$ is again an element of $H$. Moreover, $\Sigma(t)$ defines a linear operator on $H$, given as the multiplication operator $\Sigma(t)(f)=\Sigma(t) f, f \in H$. An example of a natural Hilbert space $H$ to use for modelling forward prices is the Filipovic space, which also happens to be a Banach algebra (see [5]). The detailed knowledge of the covariance operator of $Y$ (recall Corollary 27) provides a starting point for empirical analysis of the volatility and its dependency across maturities for forward prices. For a fixed time to maturity, we will have a dynamics following a fractional stochastic volatility model similar to the one in [15] as discussed above. We refer to the recent paper [1] where clear evidence of rough stochastic volatility in commodity forward markets has been found (see also [15]). In particular, they show that for front month contracts, the roughness of the stochastic volatility is in general lower than for stock markets. Indeed, the authors find empirical evidence of Hurst parameters below 0.05 for metals and below 0.15 in other commodity markets.

We can also introduce infinite dimensional extensions of the fractional Heston model. To this end, following Benth and Simonsen [8], for some $H$-valued adapted process $Z$ with $|Z(t)|_{H}=1$, define

$$
\Sigma(t):=Y(t) \otimes Z(t)
$$

Then, $\Sigma(t)(f)=\langle Y(t), f\rangle Z(t)$, and moreover, $\Sigma(t)^{*}=Z(t) \otimes Y(t)$. We take $\Sigma(t)$ as our infinite dimensional volatility process, where we observe that

$$
\Sigma(t)^{*} \Sigma(t)=Y^{\otimes 2}(t)
$$

I.e., $\Sigma(t)$ is in a sense the Cholesky decomposition of $Y^{\otimes 2}$. Notice that we use the convention $(f \otimes g)(h)=\langle f, h\rangle g$.

We are concerned with the variance/volatility of elements like

$$
U(t)=\mathcal{L} \int_{0}^{t} \Sigma(s) d B(s)
$$

where $\mathcal{L} \in H^{*}$, that is, a linear functional on $H$. If for any $x \geq 0$ the evaluation operator $e_{x}: f \mapsto e_{x} f:=f(x)$ is a continuous linear functional on $H^{3}$, we can think of $\mathcal{L}:=e_{x}$ as

\footnotetext{
${ }^{3}$ This is the case for the Filipovic space, say.
} 
the noise process of the forward contract with time to maturity $x$. In power markets, say, the forwards deliver electricity over a settlement period. From e.g. [5], one finds that $\mathcal{L}$ in this case can be represented as some integral operator which is averaging over the maturities $x \geq 0$ in some domain (corresponding to the settlement period).

The total quadratic variation of $U$ is given by the operator angle bracket process, see Cor. 8.17 in [26],

$$
\langle\langle U, U\rangle\rangle(t)=\int_{0}^{t} \mathcal{L} \Sigma(s) Q \Sigma(s)^{*} \mathcal{L}^{*} 1 d s .
$$

The instantaneous quadratic variation is the time-derivative of this expression, thus,

$$
\sigma_{\mathcal{L}}^{2}(t):=\mathcal{L} \Sigma(t) Q \Sigma(t)^{*} \mathcal{L}^{*} 1
$$

The instantaneous quadratic variation is the stochastic variance process (that is, the squared volatility) of $U$, and has the form,

Proposition 29. It holds

$$
\sigma_{\mathcal{L}}^{2}(t)=|\mathcal{L}(Y(t))|^{2}\left|Q_{B}^{1 / 2} Z(t)\right|_{H}^{2}
$$

Proof. For $\mathcal{T} \in H^{*}$, we have

$$
\left|\mathcal{T}^{*} 1\right|_{H}^{2}=\left\langle\mathcal{T}^{*} 1, \mathcal{T}^{*} 1\right\rangle=\mathcal{T} \mathcal{T}^{*} 1
$$

Hence,

$$
\sigma_{\mathcal{L}}^{2}(t)=\left|Q_{B}^{1 / 2} \Sigma(t)^{*} \mathcal{L}^{*} 1\right|_{H}^{2}
$$

By definition,

$$
\Sigma^{*}(t)(f)=(Y(t) \otimes Z(t))(f)=\langle Y(t), f\rangle Z(t)
$$

Hence,

$$
Q_{B}^{1 / 2} \Sigma(t)^{*}\left(\mathcal{L}^{*} 1\right)=\left\langle Y(t), \mathcal{L}^{*} 1\right\rangle Q_{B}^{1 / 2} Z(t)=(\mathcal{L} Y(t)) Q_{B}^{1 / 2} Z(t)
$$

The Proposition follows.

One may take $Z(t):=z$, with $|z|_{H}=1$. Thus, the stochastic variance is given as $\sigma_{\mathcal{L}}^{2}(t)=c|\mathcal{L} Y(t)|^{2}$, where $c$ is a scaling factor given by $c=\left|Q_{B}^{1 / 2} z\right|_{H}^{2}$.

Let us now look at the stochastic process $|\mathcal{L} Y(t)|^{2}$. From Theorem 26 we find that $t \mapsto \mathcal{L} Y(t)$ has paths which are $\rho=\gamma+\alpha-1$-regular, where we recall that $\gamma \in(0,1)$ is the path regularity of $W$ and $\alpha \in(0,1)$ is the fractional derivative in the Ornstein-Uhlenbeck dynamics of $Y$. Moreover, $\gamma+\alpha>1$. Denoting by $v(t)$ the expected value of $|\mathcal{L} Y(t)|^{2}$, we find from Corollary 27

$$
v(t)=\mathbb{E}\left[|\mathcal{L} Y(t)|^{2}\right]=\mathbb{E}\left[\left\langle Y(t), \mathcal{L}^{*} 1\right\rangle_{H}^{2}\right]=\left\langle Q_{Y}(t, t) \mathcal{L}^{*} 1, \mathcal{L}^{*} 1\right\rangle_{H}=\mathcal{L} Q_{Y}(t, t) \mathcal{L}^{*} 1 .
$$

Thus, the expected moments of $\sigma_{\mathcal{L}}^{2}$ is given by

$$
\mathbb{E}\left[\sigma_{\mathcal{L}}^{2 k}(t)\right]=c^{k} \mathbb{E}\left[|\mathcal{L} Y(t)|^{2 k}\right]=c^{k} \xi_{2 k} v(t)^{k},
$$

where $\xi_{2 k}$ is the $2 k$ th moment of a standard normal random variable, $k \in \mathbb{N}$. From Corollary 27, we have that $Q_{Y} \in \mathcal{Q}_{\eta}$ for $\eta<\beta+\alpha-1$ and $\beta>0$ such that $\beta+\alpha>1$. We recover a fractional behaviour in the moments of the stochastic variance process similar to what has been observed empirically for a number of assets (see [15]) and more recently for commodity forwards [1] as noted above. In our context, we have the "roughness" split into a rough noise $W$ and a fractional derivative $\alpha$ which opens for a more flexible modeling of the stochastic volatility. Moreover, we have provided an infinite-dimensional extension of the classical models. 


\section{A Fractional calculus}

We will apply certain elements from the theory of fractional calculus, involving fractional derivatives and integrals of functions taking values in a separable Hilbert space $H$. Although there are several concepts of fractional differentiation and integration, we will in this article focus on fractional calculus of Riemann-Liouville type.

Definition 30. Let $f: \mathbb{R}_{+} \rightarrow H$ be a locally Bochner integrable function in $H$, that is $f \in L_{\text {loc }}^{1}\left(\mathbb{R}_{+}, H\right)$. We define the fractional integral of order $\alpha>0$ by

$$
I^{\alpha}(f)(t)=\frac{1}{\Gamma(\alpha)} \int_{0}^{t}(t-s)^{\alpha-1} f(s) d s .
$$

For $\alpha=0$, we set $I^{0}=I$ to be the identity operator. The fractional integral $I^{\alpha}$ is a linear operator on $L_{\text {loc }}^{1}\left(\mathbb{R}_{+}, H\right)$. Furthermore, define the fractional derivative of order $\alpha$ by

$$
D^{\alpha}(f)(t)=\frac{d}{d t} I^{1-\alpha}(f)(t)
$$

where the derivative $d / d t$ is interpreted in the Frechet sense whenever this exists.

The next proposition is well known for the finite dimensional fractional Lebesgue integral, see for example [27] for an comprehensive introduction to fractional calculus.

Proposition 31. Let $f \in L_{\text {loc }}^{1}\left(\mathbb{R}_{+}, H\right)$. For any $t \in \mathbb{R}_{+}, \alpha \geq 0$ and $\beta \geq 0$, the fractional derivative and integral satisfy the following properties:

(i). $I^{\beta}\left(I^{\alpha}(f)\right)(t)=I^{\alpha+\beta}(f)(t)$.

(ii). $D^{\beta}\left(I^{\alpha}(f)\right)(t)=I^{\alpha-\beta}(f)(t)$, whenever $f \in \mathcal{C}\left(\mathbb{R}_{+}, H\right)$ and $\beta \leq \alpha$.

(iii). $\sum_{i=0}^{\infty} I^{i \alpha}(1)=\sum_{i=0}^{\infty} \frac{t^{i \alpha}}{\Gamma(\alpha+1)}=: E_{\alpha, 1}\left(t^{\alpha}\right)$.

The function $E_{\alpha, \beta}(x):=\sum_{i \geq 0} \frac{x^{i}}{\Gamma(i \alpha+\beta)}$ is known as the Mittag-Leffler function of order $(\alpha, \beta)$.

Proof. All of these identities are well known in the finite dimensional case, and we only give a sketch of the proof of their extension to the case of Hilbert valued elements here. The reader is referred to [27] for a comprehensive discussion in the finite dimensional case. First note that (i) follows from an application of Fubini's theorem for Bochner integrals, together with elementary manipulations of the fractional integral. To obtain (ii) we apply (i) together with the definition of the fractional derivative in (A.1) to write

$$
D^{\beta}\left(I^{\alpha}(f)\right)(t)=\frac{d}{d t} I^{1+\alpha-\beta}(f)(t),
$$

where the derivative is interpreted in the Frechet sense.

First, consider the case $\alpha>\beta$ with $f \in L_{\text {loc }}^{1}\left(\mathbb{R}_{+}, H\right)$. From the triangle and the Bochner inequalities, we obtain the following estimate for arbitrary $h>0$

$$
\begin{aligned}
& \frac{1}{h}\left|I^{1+\alpha-\beta}(f)(t+h)-I^{1+\alpha-\beta}(f)(t)-h I^{\alpha-\beta}(f)(t)\right|_{H} \\
& \leq \frac{1}{\Gamma(1+\alpha-\beta)} \frac{1}{h} \int_{t}^{t+h}(t+h-s)^{\alpha-\beta}|f(s)|_{H} d s \\
& \quad+\frac{1}{\Gamma(1+\alpha-\beta)} \int_{0}^{t} \frac{1}{h}\left|(t+h-s)^{\alpha-\beta}-(t-s)^{\alpha-\beta}-h(\alpha-\beta)(t-s)^{\alpha-\beta-1}\right||f(s)|_{H} d s .
\end{aligned}
$$


Since $t+h-s \leq h$ for $s \in[t, t+h]$, we find for the first integral on the right hand side that

$$
\frac{1}{h} \int_{t}^{t+h}(t+h-s)^{\alpha-\beta}|f(s)|_{H} d s \leq h^{\alpha-\beta} \frac{1}{h} \int_{t}^{t+h}|f(s)|_{H} d s .
$$

Thus, noting that the derivative of $t \mapsto(t-s)^{\alpha-\beta}$ is $(\alpha-\beta)(t-s)^{\alpha-\beta-1}$, it holds from the fundamental theorem of calculus and the dominated convergence theorem that, a.e.,

$$
\lim _{h \rightarrow 0} \frac{1}{h}\left|I^{1+\alpha-\beta}(f)(t+h)-I^{1+\alpha-\beta}(f)(t)-h I^{\alpha-\beta}(f)(t)\right|_{H}=0 .
$$

Hence, (ii) holds a.e. for $f \in L_{\text {loc }}^{1}\left(\mathbb{R}_{+}, H\right)$ when $\alpha>\beta$. If $f$ is continuous, then the fundamental theorem of calculus holds everywhere, such that $h^{-1} \int_{t}^{t+h}|f(s)|_{H} d s \rightarrow$ $|f(t)|_{H}$ for all $t \in \mathbb{R}_{+}$when $h \rightarrow 0$.

In the case of $\alpha=\beta$, the result follows from the fundamental theorem of calculus for the Bochner integral. This proves (ii). At last, from a simple computation we find $I^{i \alpha}(1)(t)=\Gamma(i \alpha+1)^{-1} t^{i \alpha}$. Thus, by summing over $i$, property (iii) holds.

Remark 32. Note that from point (ii) of Proposition 31, we see that $D^{\alpha}$ is the inverse operator with respect to $I^{\alpha}$. We further remark that we must assume continuity of $f$ to make use of the fundamental theorem of calculus for Bochner integrals.

\section{B Auxiliary proofs}

In this Appendix we have collected proofs from some of the claims in Section 5.

Proof of Proposition 23. Since $s \mapsto G(t, s)$ is integrable on $[0, t]$ and $s \mapsto Z(s)$ is continuous, the left-hand side of (5.4) makes sense as a Riemann integral. Furthermore, setting

$$
\Xi^{\tau}(t, s)=\int_{s}^{\tau} G(\tau, r) K(r, s) d r(W(t)-W(s)),
$$

where the integral term is interpreted in Bochner sense, we give meaning to the integral on the right-hand side of (5.4) as a Volterra Young integral by application of Lemma 10. To this end, we check that $L(\tau, s):=\int_{s}^{\tau} G(\tau, r) K(r, s) d r$ is contained $\mathcal{K}_{\zeta}$ for some $\zeta \in(0,1)$ such that $\gamma-\zeta>0$, and then apply Proposition 14 . Using that $G \in \mathcal{K}_{\kappa}$ for $\kappa \in(0,1)$, and $K \in \mathcal{K}_{\eta}$, it is readily checked that

$$
\begin{aligned}
\|L(\tau, s)\|_{o p} & \leq\|K\|_{\mathcal{K}_{\eta}}\|G\|_{\mathcal{K}_{\kappa}} \int_{s}^{\tau}(\tau-r)^{-\kappa}(r-s)^{-\eta} d r \\
& \lesssim(\tau-s)^{1-\kappa-\eta} \int_{0}^{1}(1-\theta)^{-\kappa} \theta^{-\eta} d \theta
\end{aligned}
$$

Due to the assumption that both $\kappa, \eta \in(0,1)$ it follows that $\left|\int_{0}^{1}(1-\theta)^{-\kappa} \theta^{-\eta} d \theta\right|<\infty$. Since $\kappa \in(0,1)$, we have that $(\tau-s)^{1-\kappa-\eta} \lesssim(\tau-s)^{-\eta}$, and it therefore follows that $\|L\|_{\eta, 1}<\infty$. By similar techniques, one can show $\|L\|_{\eta, i}<\infty$ for $i=2,3,4$, and thus we conclude that $L \in \mathcal{K}_{\eta}$ inherits the regularity $K$. Since $\gamma-\eta>0$ we conclude by Proposition 14 that the integral on the right-hand side of (5.4) is well defined in a Young-Volterra sense.

We will now show that these two integrals are in fact equal. Let now $\mathcal{P}$ denote a partition of $[0, t]$ and for some $s \in[0, t]$ let $\mathcal{P} \cap[0, s]$ be the partition restricted to the domain $[0, s]$. Then it is readily seen that following relation holds

$$
\sum_{[u, v] \in \mathcal{P}} \sum_{\left[u^{\prime}, v^{\prime}\right] \in \mathcal{P} \cap[0, u]} G(t, u)\left[K\left(u, u^{\prime}\right)\left(W\left(v^{\prime}\right)-W\left(u^{\prime}\right)\right)\right](v-u)
$$


Infinite dimensional pathwise Volterra processes

$$
=\sum_{\left[u^{\prime}, v^{\prime}\right] \in \mathcal{P}} \sum_{[u, v] \in \mathcal{P} \cap\left[v^{\prime}, t\right]}\left[G(t, u) K\left(u, u^{\prime}\right)(v-u)\right]\left(W\left(v^{\prime}\right)-W\left(u^{\prime}\right)\right)
$$

Since the left-hand side of (5.4) is a Riemann integral, it is constructed as the limit of the sum on the left-hand side above. Similarly, the integral on the right-hand side of (5.4) is the limit of the sum appearing on the right-hand side above. Since (B.1) holds for arbitrary partitions $\mathcal{P}$, it follows that (5.4) holds.

Proof of Corollary 24. An application of Proposition 23 where we set $G(t, s)=(t-s)^{\alpha-1}$ and $K(t, s)=(t-s)^{-\eta}$ (both interpreted as multiplication of scalar operators on $H$ ), reveals that for $\alpha \in(0,1)$ we have

$$
I^{\alpha}(X)(t)=\Gamma(\alpha)^{-1} \Gamma(1-\eta)^{-1} \int_{0}^{t} \int_{r}^{t}(t-s)^{\alpha-1}(s-r)^{-\eta} d s d W(r) .
$$

Next we will simplify the integral with respect to $d s$ in (B.2), and to this end, we do a change of variables by setting $s=r+\theta(t-r)$ and compute

$$
\int_{r}^{t}(t-s)^{\alpha-1}(s-r)^{-\eta} d s=(t-r)^{\alpha-\eta} B(\alpha, 1-\eta),
$$

where $B$ is the Beta-function. Using the properties of the Beta-function, we further compute that

$$
\frac{\Gamma(\alpha-\eta+1)}{\Gamma(\alpha) \Gamma(1-\eta)} \int_{0}^{t} \int_{r}^{t}(t-s)^{\alpha-1}(s-r)^{-\eta} d s d W(r)=\int_{0}^{t}(t-r)^{\alpha-\eta} d W(r) .
$$

Therefore, it holds

$$
\begin{aligned}
\sum_{i=0}^{\infty} A^{\circ i} I^{i \alpha}(X)(t) & =\int_{0}^{t}(t-r)^{-\eta} \sum_{i=0}^{\infty} \frac{A^{\circ i}(t-r)^{i \alpha}}{\Gamma(i \alpha-\eta+1)} d W(r) \\
& =\int_{0}^{t}(t-r)^{-\eta} E_{\alpha, 1-\eta}\left(A(t-r)^{\alpha}\right) d W(r),
\end{aligned}
$$

where we have applied the linearity of the integral together with the pathwise Fubini Theorem in Proposition 23 to exchange the infinite sums with integration.

Proof of Theorem 26. The proof is given in two steps. First we show the existence of a solution by showing that (5.8) satisfies (5.7), and next we show that this solution is in fact unique. An application of Corollary 24 shows that (5.8) satisfies (5.7). Indeed, define

$$
Y(t):=E_{\alpha, 1}\left(A t^{\alpha}\right) y+\int_{0}^{t}(t-s)^{\alpha-1} E_{\alpha, \alpha}\left(A(t-s)^{\alpha}\right) d W(s)
$$

Inserting (B.4) into $A I^{\alpha}(Y)(t)$ yields

$$
\begin{aligned}
A I^{\alpha}(Y)(t) & =A I^{\alpha}\left(E_{\alpha, 1}\left(A(\cdot)^{\alpha}\right)\right) y+A I^{\alpha}\left(\int_{0}^{\cdot}(\cdot-s)^{\alpha-1} E_{\alpha, \alpha}\left(A(\cdot-s)^{\alpha}\right) d W(s)\right)(t) \\
& =: I_{1}(t)+I_{2}(t) .
\end{aligned}
$$

By definition of $E_{\alpha, 1}$ (see Corollary 24), we observe that

$$
I_{1}(t)=E_{\alpha, 1}\left(A t^{\alpha}\right) y-y \text {. }
$$

Moreover, using the convolution property of the fractional integral $I^{\alpha}$ together with the representation of the Mittag-Leffler function, followed by the Fubini property from Corollary 24, we find

$$
I_{2}(t)=\int_{0}^{t}(t-s)^{\alpha-1} E_{\alpha, \alpha}\left(A(t-s)^{\alpha}\right) d W(s)-X(t)
$$


Thus, combining (B.6) and (B.7) and inserting into (B.5), it is clear that

$$
A I^{\alpha}(Y)(t)=E_{\alpha, 1}\left(A t^{\alpha}\right) y-y+\int_{0}^{t}(t-s)^{\alpha-1} E_{\alpha, \alpha}\left(A(t-s)^{\alpha}\right) d W(s)-X(t) .
$$

After rearranging this expression it follows that (5.8) solves (5.7).

We continue to prove that this solution is unique. Suppose $Y$ and $\tilde{Y}$ are two solutions in $C^{\rho}([0, T], H)$ to (5.7), starting in $y \in H$ and $\tilde{y} \in H$ respectively. Then it is readily checked that

$$
|Y(t)-\tilde{Y}(t)|_{H} \leq|y-\tilde{y}|_{H}+A I^{\alpha}\left(|Y-\tilde{Y}|_{H}\right)(t) .
$$

By application of the fractional Grönwall lemma [34, Corollary 2], Inequality (B.8) implies that

$$
|Y(t)-\tilde{Y}(t)|_{H} \leq|y-\tilde{y}| E_{\alpha, 1}\left(A \Gamma(\alpha) t^{\alpha}\right) .
$$

Thus if $y-\tilde{y}=0$, we see that $|Y(t)-\tilde{Y}(t)|_{H}=0$, which implies that the solution is unique. This concludes the proof.

\section{References}

[1] Mesias Alfeus and Christina Sklibosios Nikitopoulos, Forecasting commodity markets volatility: Har or rough, SSRN (2020).

[2] Ole E. Barndorff-Nielsen, Fred Espen Benth, and Almut E. D. Veraart, Ambit Stochastics, Probability Theory and Stochastic Modelling, vol. 88, Springer, Cham, 2018. MR-3839270

[3] Ole E. Barndorff-Nielsen and Jürgen Schmiegel, Ambit processes; with applications to turbulence and tumour growth, Stochastic Analysis and Applications (Berlin, Heidelberg) (Fred Espen Benth, Giulia Di Nunno, Tom Lindstrøm, Bernt Øksendal, and Tusheng Zhang, eds.), Springer Berlin Heidelberg, 2007, pp. 93-124. MR-2397811

[4] Fred Espen Benth, Asma Khedher, and Michèle Vanmaele, Pricing of commodity derivatives on processes with memory, Risks 8 (2020), no. 1, 8.

[5] Fred Espen Benth and Paul Krühner, Representation of infinite dimensional forward price models in commodity markets, Comm. Math. Stat. 2 (2014), no. 1, 47-126. MR-3238048

[6] Fred Espen Benth and Paul Krühner, Derivatives pricing in energy markets: an infinite dimensional approach, SIAM Fin. Math. 6 (2015), no. 1, 825-869. MR-3394359

[7] Fred Espen Benth, Barbara Rüdiger, and Andre Süss, Ornstein-Uhlenbeck processes in Hilbert space with non-Gaussian stochastic volatility, Stoch. Proc. Applic. 128 (2018), 461486. MR-3739505

[8] Fred Espen Benth and Iben Simonsen, The Heston stochastic volatility model in Hilbert space, Stoch. Anal. Applic. 36 (2018), no. 4, 733-750. MR-3854540

[9] Krzysztof Burdzy, Some path properties of iterated Brownian motion., Cinlar E., Chung K.L., Sharpe M.J., Bass R.F., Burdzy K. (eds) Seminar on Stochastic Processes, 1992. Progress in Probability 33 (1993). MR-1278077

[10] Krzysztof Burdzy and Davar Khoshnevisan, Brownian motion in a Brownian crack, Ann. Appl. Probab. 8 (1998), no. 3, 708-748. MR-1627764

[11] Giuseppe Da Prato and Jerzy Zabczyk, Stochastic Equations in Infinite Dimensions, Encyclopedia of Mathematics and its Applications, vol. 44, Cambridge University Press, Cambridge, 1992. MR-1207136

[12] T. E. Duncan, B. Pasik-Duncan, and B. Maslowski, Fractional Brownian motion and stochastic equations in Hilbert spaces, Stoch. Dynamics 02 (2002), no. 02, 225-250. MR-1912142

[13] Omar El Euch and Mathieu Rosenbaum, The characteristic function of rough Heston models, Math. Finance 29 (2019), no. 1, 3-38. MR-3905737

[14] Peter K. Friz and Martin Hairer, A Course on Rough Paths - With an Introduction to Regularity Structures, Universitext, Springer, Cham, 2014. MR-3289027 
[15] Jim Gatheral, Thibault Jaisson, and Mathieu Rosenbaum, Volatility is rough, Quantit. Finance 18 (2018), no. 6, 933-949. MR-3805308

[16] W Grecksch and V.V Ahn, A parabolic stochastic differential equation with fractional Brownian motion input, Stat. Probab. Letters 41 (1999), no. 4, 337 - 346. MR-1666072

[17] Fabian A. Harang., An extension of the sewing lemma to hyper-cubes and hyperbolic equations driven by multi parameter young fields, Stoch PDE: Anal. Comp. 9, 746-788 (2021). MR4297239

[18] Fabian A. Harang and Samy Tindel, Volterra equations driven by rough signals, arXiv:1912.02064 (2019), To appear in Stoch. Proc. Applic.

[19] Steel T. Huang and Stamatis Cambanis, Stochastic and multiple Wiener integrals for Gaussian processes, Ann. Probab. 6 (1978), no. 4, 585-614. MR-0496408

[20] Nengli Lim, Young-Stieltjes integrals with respect to Volterra covariance functions, Stoch. Anal. Applic. o (2020), no. 0, 1-18. MR-4165533

[21] Wei Liu and Michael Röckner, Stochastic Partial Differential Equations: An Introduction, Universitext, Springer, Cham, 2015. MR-3410409

[22] Terry J. Lyons, Michael Caruana, and Thierry Lévy, Differential equations driven by rough paths, Lecture Notes in Mathematics, vol. 1908, Springer, Berlin, 2007, Lectures from the 34th Summer School on Probability Theory held in Saint-Flour, July 6-24, 2004, With an introduction concerning the Summer School by Jean Picard. MR-2314753

[23] David Nualart and Samy Tindel, A construction of the rough path above fractional Brownian motion using Volterra representation, Ann. Probab. 39 (2011), no. 3, 1061-1096. MR-2789583

[24] Enzo Orsingher and Luisa Beghin, Fractional diffusion equations and processes with randomly varying time, Ann. Probab. 37 (2009), no. 1, 206-249. MR-2489164

[25] Guodong Pang and Etienne Pardoux, Functional limit theorems for non-Markovian epidemic models, arXiv:2003.03249 (2020).

[26] Simon Peszat and Jerzy Zabczyk, Stochastic Partial Differential Equations with Levy Noise, Encyclopedia of Mathematics and its Applications, vol. 113, Cambridge University Press, Cambridge, 2207. MR-2356959

[27] Stefan G. Samko, Anatoly A. Kilbas, and Oleg I. Marichev, Fractional integrals and derivatives, Gordon and Breach Science Publishers, Yverdon, 1993, Theory and applications, Edited and with a foreword by S. M. Nikolskii, Translated from the 1987 Russian original, Revised by the authors. MR-1347689

[28] Jagdev Singh, Devendra Kumar, Zakia Hammouch, and Abdon Atangana, A fractional epidemiological model for computer viruses pertaining to a new fractional derivative, Applied Math. Comp. 316 (2018), 504 - 515. MR-3703984

[29] Special-Issue, Fractional differential equations: Theory, methods and applications. Symmetry, MDPI 2019.

[30] M. Thieullen and A. Vigot, Iterated stochastic processes: Simulation and relationship with high order partial differential equations, Method. Comp. Appl. Probab. 19 (2017), 121-149. MR-3611538

[31] S Tindel, C.A. Tudor, and F. Viens, Stochastic evolution equations with fractional Brownian motion, Probab. Theory Rel. Fields 127 (2003). MR-2013981

[32] Nasser Towghi, Multidimensional extension of L. C. Young's inequality, J. Inequal. Pure Appl. Math. 3 (2002), no. 2, Article 22, 13. MR-1906391

[33] J Unterberger, Hölder-continuous rough paths by Fourier normal ordering, Commun. Math. Phys. 298 (2010), 1-36. MR-2657813

[34] Haiping Ye, Jianming Gao, and Yongsheng Ding, A generalized Grönwall inequality and its application to a fractional differential equation, J. Math. Anal. Appl. 328 (2007), no. 2, 1075-1081. MR-2290034

[35] L. C. Young, An inequality of the Hölder type, connected with Stieltjes integration, Acta Math. 67 (1936), no. 1, 251-282. MR-1555421 